The Pennsylvania State University

The Graduate School

\title{
DESIGNING LIKE A HUMAN: SIMULATING COGNITIVE STYLE IN TEAMWORK WITH AN AGENT-BASED MODEL
}

\author{
A Thesis in \\ Engineering Design \\ by \\ Samuel Lapp \\ (c) 2019 Samuel Lapp \\ Submitted in Partial Fulfillment \\ of the Requirements \\ for the Degree of
}

Master of Science

May 2019 
The thesis of Samuel Lapp was reviewed and approved* by the following:

Christopher McComb

Assistant Professor of Engineering Design

Thesis Advisor

Kathryn Jablokow

Professor of Engineering Design and Mechanical Engineering

Sven G. Bilén

Professor of Engineering Design, Electrical Engineering, and Aerospace Engineering

${ }^{*}$ Signatures are on file in the Graduate School. 


\section{Abstract}

This thesis describes the development of an agent-based model for simulating cognitive style in the context of collaborative problem solving. Cognitive style describes the diverse ways in which people solve problems. Individuals' cognitive styles can impact the success or failure of a design team. However, the effects of cognitive style in collaborative problem solving are not well understood. To address this gap, this thesis presents KABOOM (KAI Agent-Based Organizational Optimization Model), the first agent-based model of teamwork to incorporate cognitive style. In this thesis, experiments using KABOOM investigate the interacting effects of a design team's communication patterns, specialization, and cognitive style composition on a team's performance. Testing the model with a race car design problem reveals that teams can strategically leverage diversity of cognitive style to improve performance. By simulating cognitive style and team problem solving, KABOOM lays the groundwork for the development of team simulations that reflect humans' diverse problem-solving styles. 


\section{Table of Contents}

$\begin{array}{ll}\text { List of Figures } & \text { vi }\end{array}$

$\begin{array}{ll}\text { List of Tables } & \text { viii }\end{array}$

$\begin{array}{ll}\text { List of Symbols } & \text { ix }\end{array}$

$\begin{array}{lc}\text { Acknowledgments } & \text { xi }\end{array}$

Chapter 0
Mischief Managed: Lessons on collaboration from computer agents

Chapter 1

Introduction $\quad 13$

1.1 Motivation ............................. 13

1.2 Background . . . . . . . . . . . . . . . . . . . . . 15

1.2.1 The Kirton Adaption-Innovation inventory (KAI) . . . . . . . . . . . . . 15

1.2.2 Cognitive Style Diversity and Design Teams . . . . . . . . . . . . . . 15

1.2.3 Agent-Based Modeling of Design Teams . . . . . . . . . . . . . . . 16

1.3 Related Work . . . . . . . . . . . . . . . . . . . . . . 17

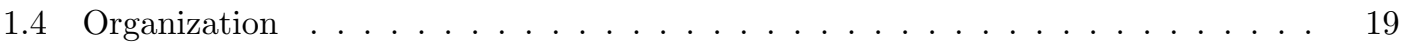

\section{Chapter 2}

$\begin{array}{ll}\text { Methods } & 20\end{array}$

2.1 Developing KABOOM ............................ 20

2.1.1 The KABOOM framework . . . . . . . . . . . . . . . . 20

2.1.2 Simulating Cognitive Style . . . . . . . . . . . . . . . . . . 22

2.1.2.1 Efficiency $(\mathrm{E}) \ldots \ldots \ldots \ldots$

2.1.2.2 Sufficiency of Originality $(\mathrm{SO})$. . . . . . . . . . . . 25

2.1.2.3 Rule/Group Conformity (RG) . . . . . . . . . . . . . 27

2.1.3 Interactions and Communication . . . . . . . . . . . . . . . . 29 
2.1.3.1 Pairwise Communication . . . . . . . . . . . . . . . 29

2.1 .3 .2 Team Meetings . . . . . . . . . . . . . . . . . . 30

2.1.4 Creating a virtual population with KAI . . . . . . . . . . . . . 31

2.1.4.1 Selecting agents for a team . . . . . . . . . . . . . . . . 31

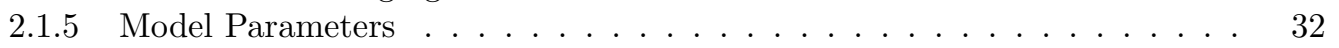

2.1.6 Evaluating Performance . . . . . . . . . . . . . . . . . . 32

2.2 Abstract Objective Function . . . . . . . . . . . . . . . . . . . . . 33

2.2.1 Objective Functions . . . . . . . . . . . . . . . . . . 33

2.2.2 Contextualized Race Car Design Problem . . . . . . . . . . . . . . . 34

2.2.3 Contextualized Beam Design Problem . . . . . . . . . . . . . . . 36

\section{Chapter 3}

Results: abstract design problems $\quad \mathbf{3 8}$

3.1 Independent Effects of Communication, Specialization, and Composition . . . . . 38

3.1.1 Communication . . . . . . . . . . . . . . . . . . 39

3.1.1.1 Pairwise Communication Frequency . . . . . . . . . . . 39

3.1.1.2 Team Meetings ....................... 41

3.1 .2 Specialization . . . . . . . . . . . . . . . . . . . . 41

3.1.3 Composition: Testing the Advantage of Style Diversity . . . . . . . . . . . 44

3.1.3.1 Performance of Homogeneous Style Teams on Diverse Problems 44

3.1.3.2 Performance of Heterogeneous Style Teams on Diverse Problems 47

3.2 Combined effects of Communication, Specialization, and Composition . . . . . 49

3.2 .1 Composition and Specialization . . . . . . . . . . . . . . . 50

3.2 .2 Composition and Communication . . . . . . . . . . . . . . 50

3.2 .3 Specialization and Communication . . . . . . . . . . . . . 52

\section{Chapter 4}

Results: contextualized design problems

4.1 Research Questions . . . . . . . . . . . . . . . . . . . . 53

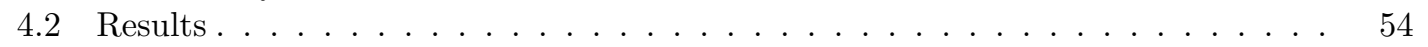

4.2 .1 Cognitive Style on Two Problems . . . . . . . . . . . . . . . . . . 54

4.2.2 Cognitive Style on Specialized Team of the Race Car Problem . . . . . . 55

4.2.3 Strategic Task Assignment Using Cognitive Style . . . . . . . . . . . . . . . . 56

4.2 .4 Modifying Problem Decomposition . . . . . . . . . . . . . . . 57

\section{Chapter 5}

Conclusions $\quad \mathbf{5 8}$

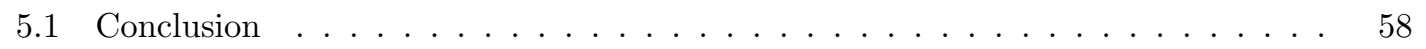

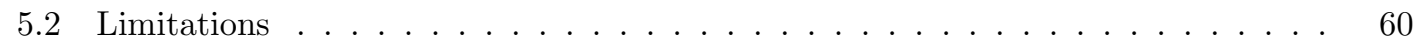

5.3 Future Work . . . . . . . . . . . . . . . . . . . . . . . . . . . . 61

5.3 .1 Six Month Time Frame . . . . . . . . . . . . . . . . . . . . . 61

5.3 .2 Three Year Time Frame . . . . . . . . . . . . . . . . . . . . . . . 61

5.3 .3 Ten Year Time Frame . . . . . . . . . . . . . . . . . . . . . . . . . . . 62

5.4 Acknowledgements . . . . . . . . . . . . . . . . . . . 63

\section{Appendix A}

$\begin{array}{lc}\text { Parameters Table } & 64\end{array}$

$\begin{array}{ll}\text { Bibliography } & 66\end{array}$ 


\section{List of Figures}

2.1 Outline of the KABOOM framework and its key features . . . . . . . . . .

2.2 Simulated annealing involves agents exploring a solution space (right) in order to maximize a defined objective function (left) . . . . . . . . . . . . . . . .

2.3 Agents with more adaptive style (lower KAI score) move in smaller steps on each iteration, while agents with more innovative style (higher KAI score) take larger steps in the solution space. . . . . . . . . . . . . . . . . . . . .

2.4 Cooling schedules for agents with different Efficiency sub-scores . . . . . . . . . .

2.5 The Efficiency sub-factor of style impacts agents risk-aversion (adaptive) or riskaffinity (innovative) of agents in solution space exploration . . . . . . . . . . .

2.6 In calculating the perceived memory location, an agent's past memories are weighted based on the serial position effect reflected in this curve: the most recent memories and earliest memories are recalled more easily than intermediate memories. . . .

2.7 The Sufficiency of Originality Effect sub-factor of style influences an agent's preference for familiar (adaptive) or novel (innovative) ideas during solution space exploration. . . . . . . . . . . . . . . . . . .

2.8 The Rule Group conformity sub-factor of style is demonstrated for homogeneous teams of adaptive (left) style and innovative (right) style. The adaptive team converges while the innovative team diverges. . . . . . . . . . . . . .

2.9 The probability of successful communication decreases as the difference in the two agents' KAI scores (cognitive gap) increases. . . . . . . . . . . . . . . . .

2.10 The objective function is a composite of a sinusoid and a parabola. The oscillation amplitude $\alpha$ of the objective function is varied from 0.22 to 5 . Global exploration of the space is important when $\alpha$ is small, but local exploitation is sufficient when $\alpha$ is large. The vertical axis shows normalized solution quality. . . . . . . . . . .

2.11 The variables in the car design problem are distributed among specialized teams. Figure adapted from Zurita et al. [1] Figure 3 and Table 1. . . . . . . . . . . . . 35

2.12 Design dimensions of $(\mathrm{A})$ a beam cross section and (B) beam loading . . . . . . . 36

3.1 Intermediate communication rates outperform high and low communication rates for 12-agent teams of randomly selected cognitive styles specialized into 4 subteams (error bars indicate \pm 1 standard deviation) $\ldots . . . . . . .$. 
3.2 This plot shows the effect of communication frequency on team performance for homogeneous teams of adaptive, mid-range, and innovative style. Innovative teams succeed with high levels of communication, while adaptive teams show little dependence on communication (error bars indicate \pm 1 standard deviation).

3.3 For small numbers of meetings, team performance increases linearly with the number of team meetings. During a team meeting, all agents converge to the best known solution. Error bars indicate \pm 1 standard deviation. . . . . . . . . . . .

3.4 Performance of teams of 32 agents with organic composition for different levels of team specialization (error bars indicate \pm 1 standard deviation) . . . . . . . . .

3.5 Plotting performance versus specialization for three homogeneous teams of different KAI styles shows that teams of innovators suffer when specializing into one or two person sub-teams while mid-range styles excel through specialization (error bars indicate \pm 1 standard deviation). . . . . . . . . . . . . .

3.6 Homogeneous teams of different KAI styles were tested on each of 25 problems (represnted by squares), with varying $\alpha$ and $\beta$. Each square's color indicates the best-performing style for that problem from blue (more adaptive) to red (more innovative). . . . . . . . . . . . . . . . . . .

3.7 Each sub-plot shows a quadratic regression of performance versus KAI score for one of 25 problem variations (as in Figure 3.6). Lower KAI scores perform best in the upper left, and higher KAI scores perform best in the lower right. . . . .

3.8 Of the 25 problems in figure 3.6, 5 were selected for each problem set. Color indicates the optimal KAI style for a problem. Problem Set 1 contains problems favoring a range of styles, while Problem Set 2 contains five problems favoring a mid-range style. . . . . . . . . . . . . . . . . . . .

3.9 Performance of linearly-distributed heterogeneous teams is plotted for problem sets 1 and 2 (error bars indicate \pm 1 standard deviation). When problems in the problem set are suited for different styles (Problem Set 1), diversity of cognitive style benefits teams. . . . . . . . . . . . . . . . .

3.10 The optimal amount of specialization depends on a team's composition of cognitive styles. Error bars show \pm 1 standard deviation in performance. . . . . . . . . . .

3.11 The optimal rate of pairwise communication depends on a team's cognitive style composition (12-agent team with 4 sub-teams). Error bars show \pm 1 standard deviation in performance. . . . . . . . . . . . . . . . . .

3.12 In these results, the best communication policy does not depend on team specialization for homogeneous teams of mid-range style. However, specialized teams perform poorly without communication. . . . . . . . . . . . .

4.1 Style did not affect performance in the beam design problem. For the car design problem, teams composed of more innovative agents performed better. . . . . . .

4.2 Varying the style of one sub-team while holding the rest of the team constant (homogeneous mid-range) revealed that depending on the sub-problem, one KAI style can perform better than others. . . . . . . . . . . . . . . . .

4.3 When a team is composed of a random selection of cognitive styles, strategically assigning agents to sub-problems based on cognitive style improves performance significantly. . . . . . . . . . . . . . . . . . . . .

4.4 Assigning variables of the car problem to sub-teams randomly resulted in better performance than using the semantic problem decomposition. . . . . . . . . 


\section{List of Tables}

2.1 List of model parameters and their default values (Objective Function parameters refer to the abstract objective function presented in Section Section 2.2 . . . .

3.1 Specialized teams decompose a problem by dividing the dimensions among sub-

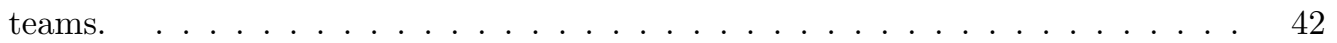

A.1 Model parameters for all results using the abstract problem. . . . . . . . . . . . 65 


\section{List of Symbols}

$K A I^{*}$ Standardized Adaption-Innovation score (re-scaled for a mean of 0 and a standard deviation of 1)

$S O^{*}$ Standardized Sufficiency of Originality sub-score (re-scaled for a mean of 0 and a standard deviation of 1)

$E^{*} \quad$ Standardized Efficiency sub-score (re-scaled for a mean of 0 and a standard deviation of 1)

$R G^{*}$ Standardized Rule/Group Conformity sub-score (re-scaled for a mean of 0 and a standard deviation of 1 )

$D$ Euclidean Distance between two solutions in the solution space

$\chi$ Random variable drawn from a Chi distribution

$s \quad$ Agent's current speed (proportional to expected step size)

$s_{0} \quad$ Agent's initial speed

$\mu_{s} \quad$ Average starting speed for all agents

$\sigma_{s} \quad$ Standard deviation of starting speed across all agents

$T$ Agent's current temperature (stochastic-ness of search in simulated annealing)

$T_{0} \quad$ Agent's initial temperature

$\mu_{T} \quad$ Average temperature for all agents

$\sigma_{T} \quad$ Standard deviation of temperature across all agents.

$\vec{x} \quad$ A vector representing a solution in the solution space.

$P_{\text {accept }}$ Probability that an agent accepts a new solution that does not improve the objective function 
$\vec{v}_{m e m}$ Agent's perceived memory direction in the solution space

$Q \quad$ Function used to calculate primacy-recency bias weights

$\Omega$ Originality, a measure of how a candidate solution compares to previous solutions

$P_{S O}$ Sufficiency of Originality preference, which affects the agent's perception of solution quality

$P_{R G} \quad$ Rule/Group Conformity preference, which affects the agent's perception of solution quality

$f_{P} \quad$ Perceived solution quality

$\vec{x}_{t e a m}$ Team position, the centroid of all team members' current solutions

$C$ Conformity, a measure of how a candidate solution conforms or diverges from team members' current solutions

$c$ Communication frequency

$\triangle K A I \quad$ Difference between two agents' KAI scores

$\alpha$ Scaling parameter for the amplitude of sinusoid in the abstract objective function

$\beta$ Scaling parameter for the size of the solution space of the abstract objective function

$R^{2} \quad$ Coefficient of determination for a linear regression

$p$ Probability (p) value for a T-test on the means of two independent samples 


\section{Acknowledgments}

I would like to thank my advisor Dr. Chris McComb for wisdom, insight, and patience with my radically innovative personality which often inhibits my ability to be an organized researcher. I would also like to thank Dr. Kathryn Jablokow for her constant support and ability to explain the concepts of personality and social science to a computer nerd. 


\section{Preface}

The organization of this thesis requires a brief explanation. This thesis is the culmination of a year of research with Dr. Chris McComb and the Technology and Human Research in Engineering Design (THRED) Group at Penn State. It begins with a chapter written in narrative form, which overviews the research conducted and principle takeaways of the research in an easily-digestible format. That chapter is intended to stand alone as a summary of my work, and is written for a wide audience. Readers looking for a traditional thesis with technical writing should feel free to skip that section, while readers looking for a lighter read should feel free to read only that section. The remainder of the thesis is written in a technical style and is comprised of material from papers I have submitted to the Journal of Design Science and the 2019 Design Theory and Methodology Conference. A Python implementation of the model I describe and test in this thesis is available free to the public on GitHub ${ }^{1}$ under the open-source MIT license.

\footnotetext{
${ }^{1}$ https://github.com/THREDgroup/kaboom/releases/tag/v1.2-beta
} 
CHAPTER 0

\section{Mischief Managed: Lessons on collaboration from computer agents}

"It's always better when we're together."

- Jack Johnson [2]

Hard leather slammed into Lasse Trockle's back as he drove the accelerator pedal to the floor. His student-built formula shot forward across the pad, reaching 60 miles an hour in 1.51 seconds. If he screamed from the rush of adrenaline, nobody heard it over the roaring engine, droning tires, and cheering team of university students. For the past year, the students were hard at work, coordinating the development of each complex component to build a formidable vehicle. Each student brought a unique set of skills, and a unique style, that was critical to the team. Somehow, they worked together to create something far beyond any of their individual capabilities.

The achievements of modern society, whether in science, engineering, international politics, or economics, are the result of great collaboration. There is something inspiring, almost mystical, about a group of unique individuals creating something more together that would be impossible alone. When Lasse's teammates designed the cabin of his racecar, they used well-established equations for air flow to predict and minimize the drag forces on the car. They could enter their design drawings in software programs that showed them where the car's geometry would cause 
more resistance, slowing it down.

But there was no equivalent tool for designing the team itself. There were no equations predicting how Eva and Steffen would perform together designing the chassis, no software programs showing which of the eight teams would progress the slowest. Instead, the team managers used their intuitive understanding of team dynamics, skills sets, and personalities to create an effective team. Across disciplines and domains, teams are created without guidance from equations or principles, and though some teams are successful, we have all been a part of a team that didn't work out so well.

\section{$\bullet \bullet$ \\ "What one programmer can do in one month, two programmers can do in two months." \\ - Brook's Law [3]}

Ten eyes gazed at me, expectantly. Crying out "I've got it!" hours into a frustratingly dry brainstorming session creates a unique flavor of anticipation. I almost regretted opening my mouth. Skeptic irony tinged the silence as my team waited for me to describe my lightbulb idea. We were designing a tool to measure the precise angles and speeds of a golfer's swing. But with the Design Showcase only a week away, our team had little to show for two months of research and design.

"Well?" intoned Sharath. I felt his exasperation, his pragmatic and industrial style yearning for a concrete plan of progress as I prepared to throw another wild curveball. The lightbulb moment for me was actually a shift from light to sound. Our fancy light sensors and imageprocessing designs had caused many headaches and slow progress. Our measurement frequency of 60 samples per second was too sluggish to track a fast-moving golf-club. I imagined a strategically placed pair of microphones listening to the golf club as it hit lightweight pegs and chimed its position to us as we recorded the audio signal 92,000 times per second. It seemed like a breakthrough.

I should have kept my mouth shut. The idea might have worked, but it broke away from all of our research on light sensors and imaging just days before our deadline. Our team splintered, with half continuing the previous prototype and half working with me on the new concept. Not surprisingly, a week later neither prototype was up to spec. I was frustrated that our team's vision was so constrained throughout the semester. I can only imagine my teammates' frustration with my propensity to wreak havoc on a plan and launch into a new, unproven direction.

That semester, any one of us could have been more successful individually than the team

was as a whole. Clashing styles and difficulty conveying our ideas to each other stunted the progress of every restart. The team was less than the sum of its parts.

During my Masters in Engineering Design at Penn State, I kept returning to the mystery 
of what makes teamwork successful. Nobody could explain why some teams struggled to perform while others found incredible synergy, becoming more than the sum of their parts. It doesn't even take brilliant individuals to create a brilliant whole: a colony of humble-brained termites works together to build a complex home with passive climate control. I found it ironic, tantalizing, that hundreds of termites could collaborate successfully, but we couldn't unlock the same synergy in a small engineering team. I began wondering if there was a secret recipe that could unlock the potential of collaborative problem solving.

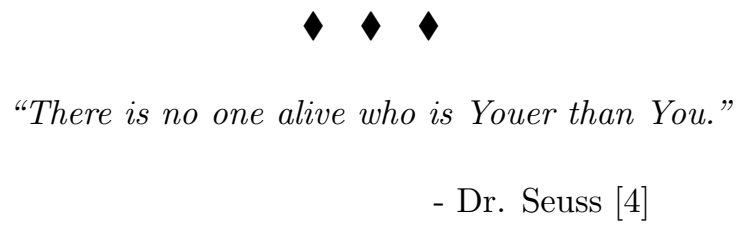

I walked into Chris McComb's office on a drizzly morning in early June of 2018 smiling and full of a different type of expectation: a sense of beginnings and open questions. The office was tidy, organized yet welcoming. A jar of pens on the table waited to be seized in a flash of inspiration. Chris smiled as I walked in, springing out of his chair to greet me with a kind handshake and curious eyes. He shared his research interests with me, a crossroads of simulation and engineering teamwork. Analyzing a dataset of thousands of engineering teams, Chris had found that individuals often outperformed collaborative groups. He wanted to know why teamwork wasn't working.

By the end of the meeting, our notes sat side by side on the table in a visual juxtaposition of our contrasting styles. Chris had inscribed neat, organized bullets on a clean notepad in a large and tidy script. A clear objective at the bottom stated a goal for the next week. My notes were scribbled at six angles and three colors on the tattered backside of a syllabus handout. Arrows and stars led the eye to a circled question mark beside an illegible line of chicken scratch.

It wasn't just our notes. Chris' style of problem solving - his "cognitive style" - is organized, precise, and methodical. He focuses on incremental progress towards a consistent larger goal. Meanwhile, I solve problems in a chaotic and disordered way, pursuing novel and radical new ideas with little regard for their quality, potential, or adherence to boundaries. As it turned out, our divergent cognitive styles became an important clue in the teamwork puzzle.

Though cognitive style describes an abstract human quality, I learned that a British scientist named Michael Kirton had studied cognitive style and developed a method to accurately measure it. Kirton was fascinated by the differences in cognitive style that made some people wellsuited for work within a large corporation (which required methodical progress and adherence to structure), while others were not. He developed an instrument, the Kirton Adaption-Innovation inventory, to measure a person's cognitive style on a continuous spectrum from Adaptive to Innovative [5].

Like my advisor Chris, Adaptors leverage existing structures to achieve careful, incremental 
progress. Adaptors abide by rules and seek consensus in a group. On the other end of the spectrum, Innovators like me seek radical and paradigm-breaking solutions with little regard for quality. Innovators ignore or actively violate constraints and norms, diverge from group consensus, and can cause discord in a team.

Team members' cognitive style can make or break a team. In his 2003 book on the topic, Kirton presented extensive research that demonstrated various implications for cognitive style on teamwork. For instance, two people with a large gap in cognitive styles can have difficulty communicating ideas and working together. In a team, style gaps can cause tension, discord, and miscommunication.

Yet other times, diversity of cognitive style can play to the team's advantage. Chris and I work together well, leveraging our polarized styles to grasp new perspectives of a problem. Unfortunately, there are no steadfast rules for when and how different cognitive styles will produce positive or negative outcomes.

Studying the effects of various cognitive styles in human teams is nearly impossible. In human studies, it is hard to isolate one variable's effects in a system as complex as a human (much less several humans). This meant we would need many trials and long experimental timeframes to draw any reliable conclusions. The magnitude of the experiments it would require to study cognitive style in teamwork was unfathomable: my two-year masters could quickly turn into centuries of human-subjects studies. I needed a shortcut.

When climate scientists want to study the earth's warming over the next several centuries, they don't conduct a centuries-long observation. Instead, they create a model that allows them to simulate centuries of global climate trends in a matter of seconds. The 21st-century solution to slow, expensive experiments is simulation: crunching the numbers of a model with powerful supercomputers. But modeling global temperatures and modeling human collaboration are two different things. Perhaps a model could simulate social interactions and team dynamics, but I didn't know of any computer that could simulate human personality and creativity.

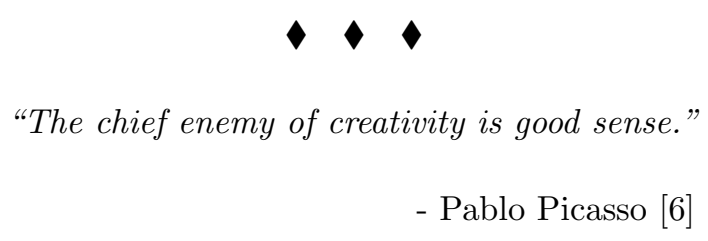

Beautiful music sounded into the control room, music that no person had ever composed. To a Baroque music lover, the intertwining lines of vocal melodies were clearly a chorale by J.S. Bach. But no Bach manuscript held these notes on yellowing 18th-century parchment. Instead, they were conceived, etched, and produced on a silicon chip smaller than a penny [7]. Whether the computer was a composer, the listeners left others to debate. It was clear to them that this digital pupil of Bach was blurring the lines of creativity, ingenuity, and innovation - lines that traditionally exclude anything non-human. 
As computational intelligence advances, computers are not only becoming better numbercrunching phenoms of logic but also gaining the ability to create and innovate in ways we consider uniquely human. In the summer of 2018, I found myself wondering if computer programs could embody the different types of creativity and problem solving that Kirton described in humans. My gut told me computers would solve problems more like Chris: methodically, carefully, and rigorously. Chris thought we could design computational problem-solvers with styles more like mine: sporadic, outside the box, and chaotic. We set our eyes on developing simulated problemsolvers spanning Kirton's entire spectrum of cognitive styles from Adaptive to Innovative.

The concept was pretty simple. But to explain our cognitive-style simulation, I need to explain the concept of an agent-based model. Say you wanted to predict the loudness of the crowd at a football stadium. The traditional model would be to come up with an equation relating some observed variables (such as the game clock, score of the game, and which team is on offense) to the loudness in the stadium. You could use historical data of the observed variables and the loudness to make your equations as accurate as possible, then predict future loudness based on the observed variables.

An agent-based model takes a more individual approach. In agent-based modeling, each individual in the crowd becomes a computer agent that decides when to scream based on the observed variables (game clock, score of the game, team on offense). Each fan makes their own decisions, but they are influenced by social interactions with their neighbors: if everyone is screaming, they join in. One fan doesn't know what every single person in the stadium is doing, just what the people around them are doing. Furthermore, each fan may be different: not everyone has the same vocal volume or the same propensity to be the first person screaming.

As you can see, the agent-based modeling approach can become very complicated and requires tracking a large number of individuals' behaviors. However, if individuals' diverse behavioral patterns and social interactions are critical to the model, agent-based modeling will capture "emergent phenomena" that a traditional model could not. Imagine, in the football stadium example, that Red Team fans have been culturally trained not to be the first person screaming, while Blue Team fans don't mind screaming alone. Because individual behaviors and social interactions are important in this case, you would want to use an agent-based model.

Agent-based modeling was a perfect fit for simulating collaborative problem solving: a group of unique individuals with limited information about their world interacting socially. Instead of fans yelling their heads off, our agents were team members working together to find the best solution to a problem. The easiest way to visualize "finding the best solution to a problem" is to imagine a topographical map where the East-West and North-South directions represent design variables (things you can change to make a better design). Any point on the map is a solution, and the quality of the solution is the altitude. During a simulation, all of the agents are running around the map trying to find the highest peak. Except that instead of the two dimensions on a geographical map, they might have to explore fifty (don't try to visualize that).

Given this framework for the model, we began giving the agents human-like behaviors. We taught them how far to move on the map in a working hour, what direction to move, and whether 
to backtrack or keep going. We taught them to share their positions with each other, and how to specialize. Each agent had a unique cognitive style on the Adaption-Innovation spectrum, and that style influenced their behaviors. But imagining exactly how cognitive style would change an agent's behavior required many thought experiments.

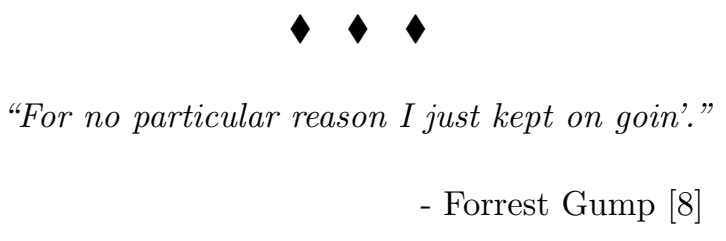

"What does your solution path look like?" I asked Chris, handing him a whiteboard marker. I couldn't describe how Adaptive and Innovative styles would translate to mathematical rules for agent behavior. Luckily, we had human subjects for Adaptive and Innovative personalities in the room: Chris and myself. Chris drew his solution path on the board, a series of connected points folding itself into a small region like a hungry caterpillar eating away at a leaf. Then I drew mine, a tangle of widely spaced points and sharp angles resembling the path of a pinball that occasionally escapes the field of play.

"I should start reading psychology," I mused.

"You need to meet Dr. J," said Chris. So we called Kathryn Jablokow, an expert on Adaption-Innovation theory and cognitive style behaviors. Dr. J was excited about the project, and started joining our meetings. The weeks would start in Chris's office, discussing how our computational agents would "prefer radical solutions", "break rules", or "conform with a group". As the agents grew more complex, Dr. J would provide examples of Adaptive and Innovative behavior to guide the model.

Sometimes human behavioral patterns fit naturally into our modeling construct. Incremental and radical solutions were clearly related to distances in the solution landscape that our agents were exploring. Other times, it felt like a stretch of the imagination to describe an agent's memory as an average of its previous solutions in that landscape, or to describe a team position as an average of the members' positions. When the conversation wandered into abstract realms, Dr. J would remind us to keep real people and their actions in mind. Eventually, the Adaptive agents started acting like Chris and the Innovative agents started acting like me. It was time to start making teams.

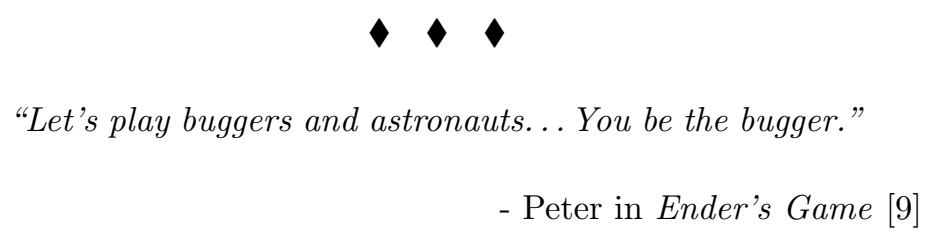

When Lasse's team designed a world-class racecar, no single team member could explain 
every aspect of the vehicle. The complex design was only made possible by dividing the problem into small, manageable sub-systems tackled by specialized teams. Problem decomposition and specialization are central to any complex design problem.

Luckily, the principle of specialized teams working on pieces of a problem fits naturally into the computational simulation framework I was working with. Picturing all of the possible designs to a problem as a map and the design quality as the altitude, you can decompose the exploration of solutions on the map into two pieces: East-West movement and North-South movement. A solo traveler would have to traverse both directions to cover the whole map, but if Lewis and Clark were exploring together they could specialize by assigning Lewis to explore East-West and Clarke to explore North-South. Of course, that might not be the smartest way to specialize. Perhaps Lewis is a better mountain-climber and Clarke is a better valley-runner. Finding the optimal problem decomposition and role for each team member is one of the central problems that a team manager faces, and one we hoped to address in our experiments.

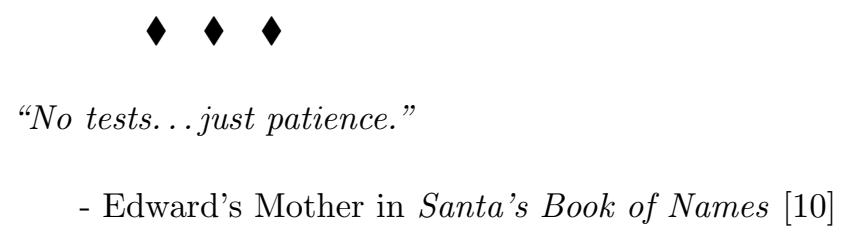

"Share with each other!" I cursed at my stubborn agents and pushed away from my desk. It was the millionth rainy day of July and I felt like a desperate preschool teacher.

"Um, are your agents misbehaving again?" asked Em from across the lab. I growled an acknowledgment. I thought that allowing agents to share their solutions with each other would unlock team synergy, but instead it was only making them worse.

Sharing solutions through communication was the central pillar of agent interaction in my model. Agents learned about each other's solutions and helped the team move forward through randomly-occurring one-on-ones where they shared their deepest secrets (that is, mostloved solutions). If they preferred to keep their secrets to themselves, the team just became a collection of individuals working on their own.

I tried everything I could think of to encourage my bratty little agents to share with each other. What worked, in the end, was patience. They needed more time: literally, more frames in the simulation to make communication a valuable activity. Then, the silicon collaborators started behaving remarkably like real teams.

My experimental knob was the fraction of work hours in an agent's life that they spend communicating, instead of exploring new solutions independently. Studies of real human teams found that as the amount of communication rose from zero, the team's performance increased up to a point, then leveled off and eventually decreased as the amount of communication became excessive [11]. Incredibly, as soon as I extended the simulation time frame, my model produced the same results. There was a curved communication trade-off where an intermediate amount of 
communication produced the best performance.

By mid-September, my agents had unnervingly human behaviors for communication and specialization. Best of all, the agents embodied a range of cognitive styles ranging from evenmore-Adaptive than Chris to even- more-Innovative than me. Though the agents weren't perfectly human (thankfully), it was time to put the model to the test.

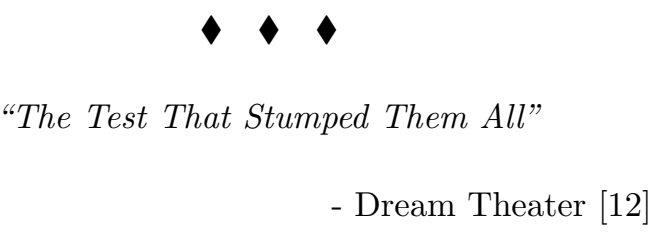

In my previous experiment on communication (and in the study that it mimicked) the teams contained a random composition of cognitive styles. When we simulated teams where all of the agents had the same cognitive style, strange things started to happen. A team composed of only Adaptors seemed to require only a fraction of the communication that other teams needed. A team of only Innovators performed best if they spent almost all of their time communicating, and only a fraction of their time problem solving.

Unsure if these patterns would actually occur in real teams, I scoured the limited scientific literature on cognitive style in teams. I was surprised to find an observational study from 2015 with similar results to our computational experiment. When Gianluca Carnabuci and Blint Diszegi studied social networks in product development teams, they found that Adaptive individuals were more successful in networks with limited connections while Innovative agents were more successful in densely connected networks [13]. It wasn't crazy, then, that our Adaptive agents preferred low levels of communication while Innovative agents wanted to communicate practically all the time.

\section{$\bullet \bullet$ \\ "In diversity there is beauty and there is strength." \\ - Maya Angelou [14]}

In kindergarten, we started learning how to get along with people who are different from us. It is a life-long learning process. The teams and groups that we are a part of won't contain carbon-copies of us, and sometimes our personalities clash. In my Master's program Golf Project team, wide chasms in style made teamwork painful and difficult. Yet, sometimes diversity can be the greatest strength of a team.

Lionel Messi may be the greatest footballer of all time, but eleven Messi's wouldn't make a strong team. And unlike skill level, Dr J reminded me, cognitive styles are not 'better' or 'worse', they're just 'different'. For Lasse's race car team, individual differences were the ingredients for 
a successful recipe. But how to use team members' unique styles as an advantage, instead of a headache, is anything but clear.

To address this mystery, I first needed to investigate the strengths and weaknesses of each style. In the computer world, we could modify the landscape of a problem by making the map bigger or smaller, or by changing the severity of the hills and valleys. I found that on a small map with extremely sharp peaks and valleys, Adaptors outperformed other styles. On huge maps with rolling hills, Innovators had an advantage. The best-suited agents for intermediate landscapes had styles between extremely Adaptive and extremely Innovative.

Then, I started giving the simulated teams multi-day challenges. Each day, the team would tackle a landscape, trying to find the best solution to the problem by searching for the highest altitude. I pitted two types of teams against each other: Uniform teams contained agents of the same mid-range style, but Diverse teams had huge ranges of cognitive style from Adaptive to Innovative.

And the Cognitive Olympics began. When all five days of the trial featured similar landscapes, there was no difference in performance: on average, Uniform and Diverse teams had the same solution quality. But when each of the five days featured a different terrain, the Diverse teams crushed the Uniform teams in overall performance. Team diversity only became advantageous when they faced a diverse set of problems. We hadn't unlocked the secret to leveraging diversity, but we had found one more clue.

\section{$\bullet \bullet$ \\ "You still speak in riddles." \\ - Aragorn in The Two Towers [15]}

"What does this mean, in real life?" Dr. J asked me one dreary November morning.

"Nothing at all," I sighed heavily. By late Fall, living in the abstract space of a computer simulation had turned my visual mind into brain stew. We could run simulations all day long, with my little agents running around a landscape of fifty-dimensional hills and valleys, but I had no idea what it meant if they ended up at the top of a fifty-dimensional mountain.

We had discovered that Adaptive agents performed best in landscapes crisscrossed with deep crevices, whereas Innovative agents performed best in mountainous, Himalayan topographies. But the problems were not tied to any physical reality, any real problem. The solutions and their scores were purely mathematical: they couldn't be measured in meters or dollars. I needed to bring the model back to earth. Back to the race track with Lasse Trockle, his roaring formula, and a team of university students.

"So, let's have them design cars," said Chris. He wanted the agents to design a Formula SAE race car, just like the student team, instead of designing an arbitrary vector in a mathematical hypercube. It would let us tie physical meaning to variables like tire radius, sus- 
pension height, or cabin density. Even better, we would understand the design objectives as real outcomes: acceleration, braking distance, turning radius.

The race car was a behemoth-problem compared to the collection of sine-waves and parabolas that we had been using. Now we had constraints, physical dynamics, and interacting variables. Some variables - like the cabin's width - were continuous numbers, but others - like the cabin material - were not. It was a mess.

We were lucky to be following in the footsteps of Nicholas Zurita, a Graduate Research Assistant at Oregon State University. Zurita had already implemented the race car design problem for an optimization model [1] and held my hand through the process of translating the design of a race car into a language that computer agents could understand.

The added realism of the problem came at a heavy price. Compared to an abstract problem, simulations with the race car problem took a couple hundred times longer to run. But at least we could have some semantic connection from the simulation to real life. Some agents designed the wheels while others designed the cabin. Heavier was worse, faster was better. It made some intuitive sense.

As a sanity check, I ran the same communication frequency study from the abstract problem on the race car design problem. The new model passed with flying colors. As expected, intermediate communication frequencies were superior to low and high rates of communication. I felt like I had my feet back on solid ground, and it was time to start answering some of our questions about cognitive style and teamwork.

\section{$\diamond \diamond$ \\ "... You missed the starting gun" \\ - Pink Floyd [16]}

Wheels spun in place, digging themselves into a muddy puddle as the engine revved up to $4000 \mathrm{rpm}$. At least, that's how my brain felt with hours left before a conference submission deadline in late February. I was finishing a paper that had come together in a five-day flurry of computational experiments, high-speed writing, and excessive coffee. The writing was practically complete, but the paper felt stagnant, underwhelming, like a race car revving its engine but never shifting into gear.

Using the new race car design problem, I wanted to demonstrate the advantage of cognitive style diversity in specialized teamwork. The abstract Cognitive Olympics had given us an important clue: teams can leverage diversity of cognitive styles when they face a range of problems that are best suited for different styles.

In the race car problem, specialized teams tackled different parts of the car such as the suspension, engine, and impact attenuator. I discovered that the most effective cognitive styles varied among these pieces of the design problem: Adaptive agents were most effective for the 
suspension; Mid-Range agents for the engine; and Innovative agents for the impact attenuator.

Then I created dream-teams. Like a football team manager with a bottomless wallet, I drafted a team with the ideal agents for each specialized task. It was no surprise when these dream-teams crushed the teams with members randomly selected off the street.

But it felt like cheating. The student race car team couldn't afford to simply buy the best motor, best, suspension, best everything: they had to work within a budget. The same should be true of team members: a manager doesn't get to hand pick the best people for his team, but has to work with the group they have. Given a team of random off-the-street members, I thought that a manager should be able to strategically align their cognitive styles with their tasks. I was playing manager, but none of my strategies were improving performance. My wheels were spinning, and it was too late to win the race. I sent the paper to Chris for final revisions, not satisfied with our results but out of time.

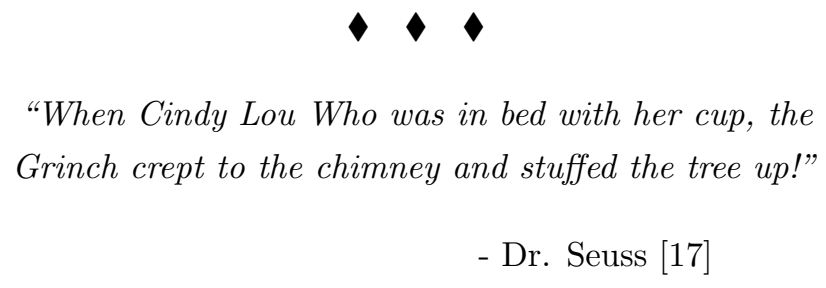

Like the Grinch, I had unscrewed one light bulb and it made the whole Christmas tree go dark. And, like the Grinch, I used the cover of night to steal the tree away, fix it up, and return it triumphantly in the early morning light.

I knew Chris was in bed, and that gave me one more chance to figure out why my strategies for leveraging cognitive style diversity weren't improving the performance of my computational teams. I found a mistake in my code. It was a small error, a few characters, but like a faulty light on a Christmas tree its effects cascaded into a chain of darkness. As I fixed it, I knew even before running the simulation that I was shifting out of neutral and into gear.

The performance was phenomenal. I took a team with random, off-the-street cognitive styles and assigned each agent to a task that suited their style. They performed incredibly: just as well as the dream-teams of hand-picked agents. À la Grinch, I popped my eyes. Shoving large pieces of my current draft up the chimney, I rewrote the central pieces of the paper before Chris woke up the next morning.

You might wonder why I was so excited. It's no big surprise that assigning team members to the right tasks is important. You wouldn't put a structures engineer on the sales team for a new high-rise, or a salesperson on structural design. Domain knowledge and expertise are critical factors for the manager to consider.

But our simulation knew nothing about domain expertise: one agent knew as little as another about designing any part a car. My strategic team assignment was based exclusively on the agents' problem solving styles. You could never isolate cognitive style from knowledge in a 
human study, but our simulation demonstrated that cognitive style has its own importance when assigning team members to tasks. It showed that a team could turn the diversity of its members' cognitive styles into a strategic advantage for tackling a complex problem.

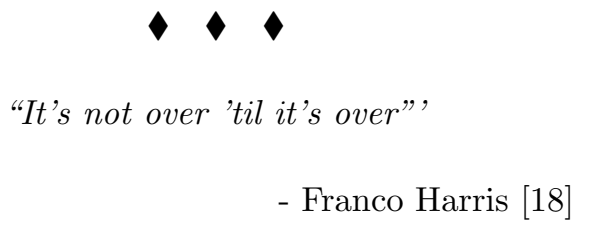

Teamwork is likely to remain a compelling mystery for a long time. Unlike the drag on a race car's cabin, the success of a team can't be reduced to a rule, equation, or even an agent-based simulation. In building and testing this simulation, I have peeked into the vast complexity of humans as problem solvers and social beings. If anything, I'm less sure now than ever that I can describe how a person works in a team to solve a problem.

The reason is that every person is unique and unpredictable. Our differences - our unique approaches to perceiving and responding to the world - are rich and colorful. Sometimes, people like Kirton find a way to describe one aspect of our differences in a concrete way, offering us an immensely useful tool for studying those differences.

Like a 2-dimensional map of a 50-dimensional problem, we are only looking at a slice of the personality landscape. Still, the map might help us understand a new aspect of human behavior, and design better teams. At the very least, our computational experiments demonstrate that cognitive style really matters in teamwork. The styles of problem solving and social interaction that Kirton quantified into the Adaption-Innovation spectrum play an important role in a team's success. Managed strategically, diversity of cognitive style can play to a team's advantage.

Simply recognizing the differences in style between myself and my collaborators has given me a capacity for empathy and conflict-resolution that I didn't know I was missing. I can't state a rule that will avoid clashing styles like my Golf Project team, or guaranteed synergy like my collaboration with Chris. But by paying attention to cognitive style, along with other facets of individual skill and personality, you will have one more tool for making teamwork work. You might also gain a new lens to recognize and celebrate the unique colors that each person offers to a team, and to the world. 
CHAPTER 1

Introduction

\subsection{Motivation}

Current design research frequently draws conclusions based on small scale behavioral experiments. Though valuable, these studies are severely limited in scope, and the results are difficult to generalize. The future of design cognition analysis will increasingly need to leverage computational methods for modeling and analyzing both individual and team behavior to enable larger scale studies. In addition, design cognition research must continue its trend toward more rigorous modeling of individual cognitive differences among designers to support more accurate simulations; cognitive style, or one's preference for managing structure in solving problems, is one key example. In general, cognitive style describes patterns in problem-solving behavior and social interactions that result from an individual's unique cognitive processes. For example, one measure of cognitive style differentiates verbal and visual learners [19], while others distinguish more adaptive and more innovative thinkers [5]. Cognitive style varies widely across engineers and designers, and differences in cognitive style can have positive or negative effects on the problem-solving performance of an individual or team [5, 20, 21, 22]. While researchers are beginning to develop simulation approaches for studying team problem solving, current methods do not yet attempt to model the influence of cognitive style on individual and team activities. To address this gap, I present in this thesis an agent-based model for assessing how individuals' cognitive styles impact an engineering team's problem-solving performance, thereby linking cognition, behavior, and design activity.

Cognitive style is assessed using psychometric instruments such as the Kirton Adaption- 
Innovation (KAI) inventory [23], which measures an individual's relative preference for structure along a bipolar continuum between two equally valued extremes (highly adaptive and highly innovative, respectively). More adaptive individuals prefer more structure in their problem solving (with more of it consensually agreed) and therefore tend to make incremental improvements within the current system to improve and enhance it. In contrast, more innovative individuals prefer less structure, with less concern about consensus, and tend to make radical changes that may ignore rules in an attempt to make the current system work "differently"; their efforts may or may not lead to improvements in that system. Kirton's A-I cognitive style influences both individual problem-solving characteristics and team interactions, and its effects have been studied in observations of engineering teams $[22,24,25,21]$. However, it is unclear how KAI style could be used to inform the formation of engineering teams.

In fast moving projects, team formation is a critical task that managers must perform using their best guesses about optimal team composition and structure [26]. As engineering teams become more interdisciplinary and develop increasingly complex structures, selecting the right members for a team is becoming more difficult as well [27]. However, research on optimal team formation strategies is limited. Research on collaboration in team performance is often based on qualitative descriptions and small studies [28]. In vivo studies of design teams over long periods of time are expensive, and the results are often applicable only in a specific context [29].

In light of these issues, agent-based models of team problem solving can serve as a powerful tool for design cognition analysis, because they can quickly compare many different team compositions, structures, and processes [27]. Also, simulation can be useful for isolating independent variables in studies of cognition or social interaction [30, eg], which is difficult in a human-subjects study. Current methods in design cognition analysis can simulate team problem solving involving agent communication [31, 29, eg], sometimes with learning mechanisms [32, 33, eg] and social interaction $[34,30]$, and for some real-world design problems [1, 26, eg]. However, none of these methods address the role of cognitive style in problem solving and team performance explicitly.

This thesis presents the KAI Agent-Based Organizational Optimization Model (KABOOM), the first agent-based modeling framework for studying the problem-solving performance of teams of individuals with diverse cognitive styles. In KABOOM, individual agents exploring a solution space in order to maximize an objective are used to model human problem solving. Each agent has a unique cognitive style to reflect the range of styles measured by the Kirton AdaptionInnovation inventory. An agent's cognitive style influences its exploration and evaluation of the solution space. The interaction and collaboration between agents also depends on the agents' respective cognitive styles. The goals of this research are (1) to construct an agent-based model with agents that reflect the diverse cognitive styles of humans across the Adaption-Innovation spectrum; (2) using the model, analyze the performance of individual agents and teams solving a design problem; (3) investigate how cognitive style impacts the effects of team specialization and communication on performance; and (4) investigate how teams can leverage diversity of cognitive style to improve performance. After reviewing related work in Adaption-Innovation theory and agent-based modeling, this thesis describes the development of KABOOM and discusses the re- 
sults of several computational experiments on team specialization, communication, and cognitive style composition.

\subsection{Background}

\subsubsection{The Kirton Adaption-Innovation inventory (KAI)}

The Kirton Adaption-Innovation inventory (KAI) is a psychometric instrument designed to measure a person's cognitive style on a continuous spectrum that ranges from highly adaptive to highly innovative [Kirton 2003]. In general terms, more adaptive problem solvers aim to do things better by using incremental changes to continuously improve a system or solution. In contrast, more innovative problem solvers prefer to do things differently, pursuing radically different solutions with more regard for originality than quality. Innovators tend to challenge the existing structures and constraints of a problem, while adaptors tend to support and stay within preexisting structures and bounds.

The KAI has 32 items, each answered on a 5-point scale [23]. In addition to an overall KAI score, the instrument provides three sub-scores: Sufficiency of Originality (SO), Efficiency (E), and Rule/Group Conformity (RG) [23] that are related to different aspects of cognitive style. The Sufficiency of Originality sub-score relates to the quantity and paradigm-relatedness of the solutions a person generates. More adaptive individuals tend to generate fewer ideas (based on cognitive preference, not ability); these tend to be paradigm-preserving and are easier to integrate into existing systems. More innovative individuals tend to generate a higher number of solutions (again, based on preference, not capacity); these offerings tend to be more paradigm-breaking and can be difficult or even harmful to integrate into the existing system [23]. The Efficiency subscore describes an individual's attention to detail and methodological approach. More adaptive individuals prefer incremental changes to a solution that are sure to improve quality. In contrast, more innovative individuals may alter a solution in riskier, less well-defined ways, with less regard for the resulting quality [23]. The Rule/Group Conformity sub-score describes an individual's preference for adhering to constraints and norms, as well as their cohesion with a group (i.e., social structure). More adaptive individuals prefer to leverage the prevailing rules, guidelines, and norms in their problem solving; they also tend to promote group cohesion and continuity. More innovative individuals, however, are more likely to disregard the prevailing rules and norms, and are less concerned about conforming to a group; they may actively move away from group consensus and can cause disruption or discord in a team [23]. The methods section describes how each of these sub-factors of KAI inform the development of agents that have their own cognitive style.

\subsubsection{Cognitive Style Diversity and Design Teams}

When individuals work together, their diverse cognitive characteristics can influence their col-

laborations in both positive and negative ways. Kirton uses the term cognitive gap to describe 
differences in cognitive level (intelligence, experience, knowledge, etc.) and/or cognitive style that can appear between two individuals, an individual and a group, two groups, or between an individual/group and the problem at hand [5, 24]. In previous work related to KAI and teams, Kurtzberg [35] studied the creative fluency of homogeneous teams and heterogeneous teams (as categorized by KAI cognitive gaps) and found that heterogeneous teams outperformed homogeneous teams in terms of creative output (i.e., number of ideas). Hammerschmidt [36] studied team success and cognitive style diversity using KAI and found that teams had higher levels of success when tasks were coordinated with KAI (e.g., a more adaptive task aligned with a more adaptive sub-team). His work also revealed that when sub-teams had similar KAI scores (i.e., were homogeneous), overall team success increased as a result of enhanced inter-team communication, while diverse (i.e., heterogeneous) teams were more likely to fail as a result of unresolved cognitive gaps. Jablokow et al. [21] explored the effects of cognitive gaps on dyad performance and interactions between design team members during concept generation. Their results suggest that as the cognitive gap between team members increases, the more adaptive team member tends to feel that they contributed less to team ideation, while the more innovative team member tends to feel that they contributed more. In other previous work by Jablokow et al. [21], the results suggest that the presence of more innovative individuals on a team may be correlated with a greater occurrence of unique ideas in the team, where unique was defined as new to the current team discussion. They also found that more innovative teams (by KAI mean) tended to exhibit team interactions with a higher degree of integration between topics.

\subsubsection{Agent-Based Modeling of Design Teams}

An Agent-Based Model (sometimes called a Multi-Agent System) is a software simulation method in which autonomous, heterogeneous agents interact with their environment and other agents [37]. Each agent acts with a set of behavioral rules in order to accomplish an objective [38]. Rather than defining the macroscopic behavior of a system, an agent-based model only defines behavioral rules for individual agents and a structure for agent interactions [38, 39]. Agents make run-time decisions based on limited knowledge of the environment and limited decision-making capabilities [39,34]. In addition to these cognitive constraints, agents may be socially constrained with limited ability to connect and share information with other agents [34]. By simulating agent interactions, an agent-based model can capture emergent phenomena that are more than the sum of the individual agents' actions [38].

Some studies investigate the frequency of communication among agents throughout the course of the problem-solving period. In the context of design research, communication refers to the exchange of design representations, in other words solutions [30]. Research on communication in design teams shows that more communication is not necessarily better; there is a trade-off between communication frequency and individual work [11, 40]. Some researchers have shown that intermittent communication can provide the benefits of constant communication, as well as the benefits of individual work [41]. There are even some cases where no communication is the 
best team process strategy $[42,43]$.

Engineering teams often divide the design of complex systems into multiple components that are then addressed or solved by specialized team members with corresponding expertise in a specific domain $[32,44]$. This multi-component approach to complex systems design is analogous to coordination between multiple agents in a computational model: decisions are made with respect to separate components and later aggregated to form a global solution [1]. An agentbased model can reflect the way engineering teams solve complex systems in a distributed manner by assigning agents to specialized sub-teams that each control a subset of all design variables for

a global objective function [44]. This approach makes it possible to use an agent-based model to rapidly investigate the effects of team structure on a design team's performance.

Appropriate team composition is critical for high team performance [45] and should consider not only the personal traits of individuals, but also the nature of the problem being addressed by the team [46]. Team composition can have a detrimental effect on performance and social interactions if teams are not carefully constructed and managed [45]. Although this thesis examines team composition in terms of the cognitive styles of agents on a team, other factors of team composition exist and have been studied both in human subjects studies and through computational modeling. According to Singh et al. [30], a team's composition refers to its size, life-span (one project or several), location (local or geographically distributed), structure (flat or hierarchical), and heterogeneity (homogeneous or heterogeneous). Not surprisingly, a team's composition in terms of domain of expertise also plays a key part in product development success [47]. However, there is little evidence regarding how other personal cognitive qualities like cognitive style influence a team's success. Martinez-Miranda and Pavon Martinez-Miranda2012 state that although some human resources departments use tests of personality and cognitive level:

It could be even more useful for project managers to apply the results of cognitive and psychological tests to build virtual teams and simulate their possible behaviors in order to analyze what could happen when people with specific characteristics interact with each other and with their respective tasks over the entire duration of a project. $[45]$

Among the large number of psychological instruments used today, many have limited validation or scientific support. The Kirton Adaption-Innovation inventory (KAI), however, has been extensively validated and is regularly used for team management [5]. This research, therefore, aims to fill a gap at the intersection of cognitive style and agent-based modeling by incorporating Kirton's Adaption-Innovation cognitive style characteristics into an agent-based model of engineering teamwork.

\subsection{Related Work}

In the 1990s, managers of the semiconductor manufacturer "Micro" were faced with the challenge of developing high-performance teams for complex and concurrent projects on an extremely rapid 
product life cycle [26]. While the performance of their semiconductor chips could be modeled with computer simulations, managers still used guess-and-check methods for the design of their teams - but they began to ask for computational tools that could simulate team performance in the same way that software simulations could predict the performance of an engineering design [26]. This led to the Virtual Design Team (VDT) project $[48,26]$, an early effort to create simulation tools that helped managers design software teams by simulating the assignment and completion of work items. The VDT simulation divides a problem into tasks and types of work, and then uses stochastic discrete event simulation to model the time and number of errors incurred in completing each task. VDT models real and complex projects, and includes both errors in task completion and noise in communication [26].

Since then, many researchers have developed models with the aim of reflecting some aspect of human behavior or teamwork [49, eg] more accurately. These models vary in their purpose. Some, like VDT, are team management simulation tools that model team performance to avoid the associated costs of long and expensive human subjects studies [29, eg]. Other models focus on studying the social aspects of communication and collaboration [30, 34, eg]. For example, Tsvetovat and Carly [34] develops a detailed model of complex social and technological systems by including learning, social network theory, and social psychology in an agent-based model. Fan and Yen [31] present an extensive review of agent-based models addressing communication and collaboration; some of the models reviewed include emotion and sentiment, but none reflect individual cognitive style [31].

Recently, several agent-based models have attempted to focus on the personalities, attitudes, and emotions of problem solvers, as well as their knowledge [50, 46, 51, eg]. For example, the TEAKS framework $[50,45]$ models the social and emotional aspects of team problem solving. Agent interactions are based on the PECS (physics, emotion, cognition, and social status) framework [52], and personality is described by drive, influence, steadiness, and compliance. Dehkordi et al. [51] studies work-overload impact by modeling the effects of stress and motivation on team performance, and Zhang et al. [46] presents a model where human behavior, development process, and organizational structure all influence design outcomes. These models focus on emotions and sentiments to elicit human-like behaviors in computational agents.

Finally, some models implement contextualized, real-world design problems that are solved by teams of agents in an agent-based model [1,33, eg]. Work by Zurita et al. [1] is an example of an agent-based model specifically created for a contextualized problem: designing a Formula SAE racing vehicle. Their model demonstrates that using a cooperative evolutionary algorithm, an agent-based model can design a complex system. Like Hulse et al. [44], members of the design team adopt specializations by breaking the problem into separate functional subsystems (e.g., engine, suspension, and brakes).

The CISAT framework $[33,53,42]$ is another agent-based model which uses contextualized problems to study how problem characteristics affect the optimal team process and team characteristics. The CISAT framework reflects eight characteristics of both team activity and individual cognition, namely: organic interaction timing, quality-informed solution sharing, quality bias re- 
duction, self-bias, operational learning, breadth versus depth solution search, and satisficing [33]. In McComb et al. [42], the CISAT model is used to find optimal team characteristics based on properties of the problem being addressed. These two models provide much of the inspiration for KABOOM, the agent-based model presented in the following chapter.

\subsection{Organization}

The remainder of this thesis describes the methods, results and future work related to KABOOM. Chapter 2 describes the the agent based model KABOOM, the implementation of cognitive style for agents in the model, the creation of a virtual population of agents with distinct cognitive styles, and the implementation of objective functions for one abstract problem and two contextualized problems.

Chapter 3 discusses the results of experiments with KABOOM that use the abstract objective function. The first half of the chapter studies the independent effects of communication, specialization, and team cognitive style diversity on team performance for agents with various cognitive styles. The second part of Chapter 3 investigates interactions between communication, specialization, and style composition. The work presented in Chapter 3 was submitted as a journal article to a thematic collection of the Journal of Design Science called "The Future of Design Cognition Analysis".

Chapter 4 uses two contextualized design problems (a race car design problem and a beam design problem) to study how different aspects of a problem can be best suited for different styles, and demonstrates how a team can strategically use cognitive style diversity to improve performance. The work presented in Chapter 4 was submitted as a conference paper to the ASME 2019 International Design Engineering Technical Conferences and Computers and Information in Engineering Conference.

Finally, Chapter 5 summarizes the major findings of this research, describes limitations of the model, and suggests directions for future work related to this thesis in six month, three year, and ten year time frames. A Python implementation of the model presented in this thesis is available free to the public on GitHub ${ }^{1}$ under the open-source MIT license. Please reach out to me through email ${ }^{2}$ if you have questions regarding the model or research described in this thesis.

\footnotetext{
${ }^{1}$ installation: pip install git+http://github.com/THREDgroup/kaboom.git@v1.2-beta

${ }^{2}$ sam.m.lapp@gmail.com
} 
CHAPTER 2

Methods

This chapter first describes the development of the agent-based model KABOOM, then explains the implementation of two contextualized problems for the model.

\section{$2.1 \quad$ Developing KABOOM}

KABOOM is the first agent based model to incorporate cognitive style of heterogeneous agents as a strategy to simulate the problem-solving behaviors of human teams. This section describes (1) the agent-based model framework for KABOOM, (2) the implementation of Kirton's Adaption-Innovation cognitive style for agents, (3) communication in the model through pairwise solution sharing and team meetings, and (4) creating a virtual population with KAI cognitive style traits. Figure 2.1 provides a high-level summary of the key components of the model.

\subsubsection{The KABOOM framework}

The KAI Agent-Based Organizational Optimization Model (KABOOM) is a multi-agent optimization scheme in which independent software agents explore a solution space by varying parameters and evaluating a cost function. KABOOM is based on a simulated-annealing [54] optimization algorithm: the agents start with a high temperature, allowing them to explore widespread solutions non-greedily; the temperature gradually cools over the course of the sim-

ulation, leading to a local and more greedy search. The gradual transition from stochastic to downhill search in simulating annealing reflects the nature of human problem solving $[55,56]$ 


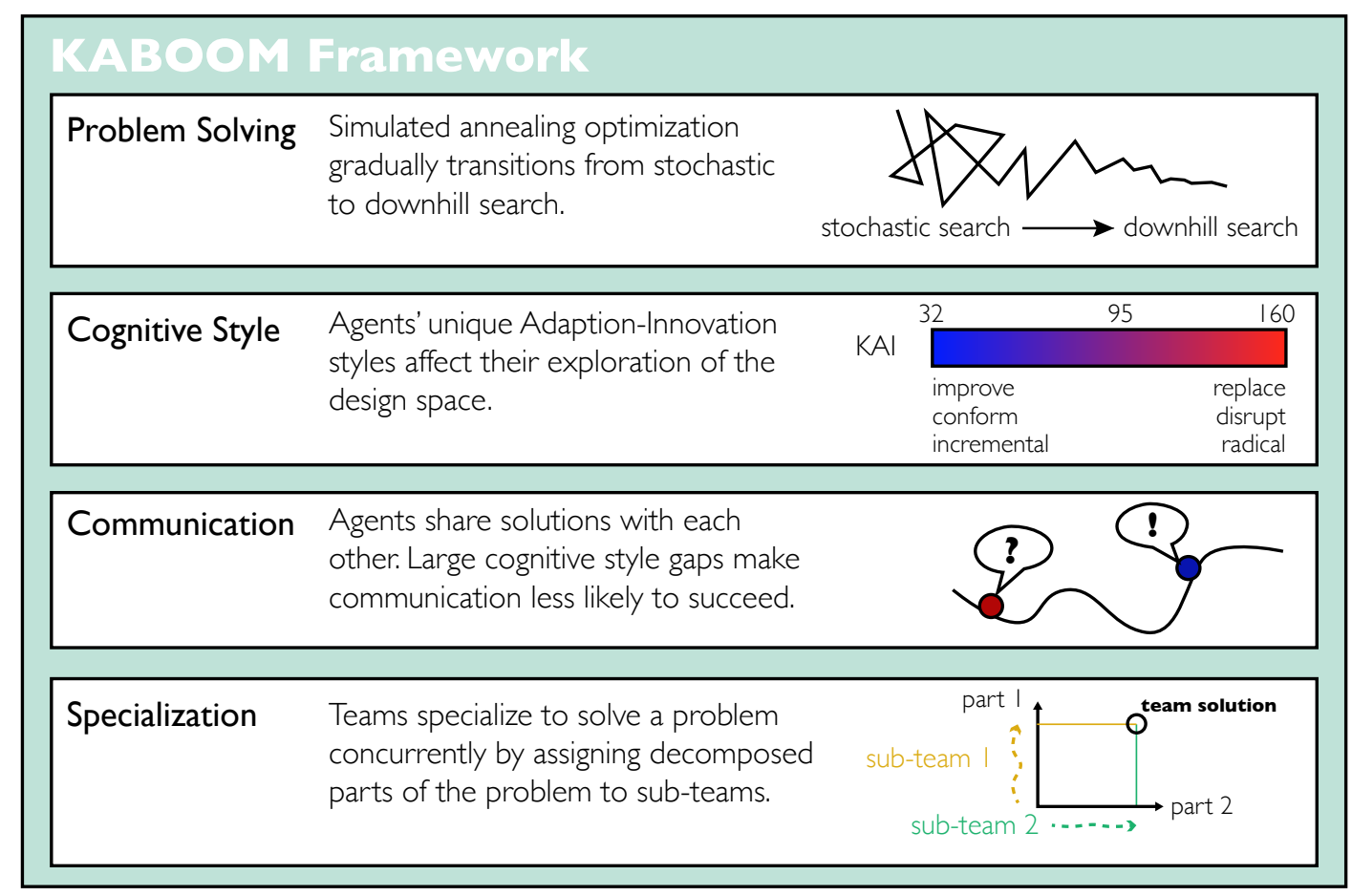

Figure 2.1. Outline of the KABOOM framework and its key features

and has been used in other human problem-solving simulations [33, eg]. Figure 2.2 illustrates an agent problem solving in a simulated annealing framework. The agent explores a 2-dimensional problem space with the goal of maximizing an objective function (left). The black line connects the sequence of solutions explored by the agent over the course of 100 iterations of the simulation, ending at the black diamond. The agent makes large changes to the solution early on, followed by smaller steps towards the end, in accordance with the simulated annealing scheme.

Agent-based models are most useful for modeling individual and group behavior when agents behave like humans, rather than behaving optimally in the sense of maximizing an objective function [34]. Because of this, agent behaviors and model parameters are tuned to reflect observed and theorized human behavior as much as possible, rather than to achieve optimal performance.

Many computational models of team problem solving assume that agents can perfectly evaluate the objective function for solutions they create. This is due in part to agent-based models being grounded in the optimization literature. In contrast, KABOOM assumes agents can evaluate the quality of their solutions, but their perception of solution quality is affected by their respective cognitive style. Each agent has an assigned cognitive style, which includes the three sub-score dimensions of Sufficiency of Originality (SO), Efficiency (E), and Rule/Group Conformity (RG), in addition to the total score (KAI). These parameters influence how the agent perceives solution quality and how it explores the solution space. The following sections describe how the total KAI score and each sub-score impact agent behavior. 

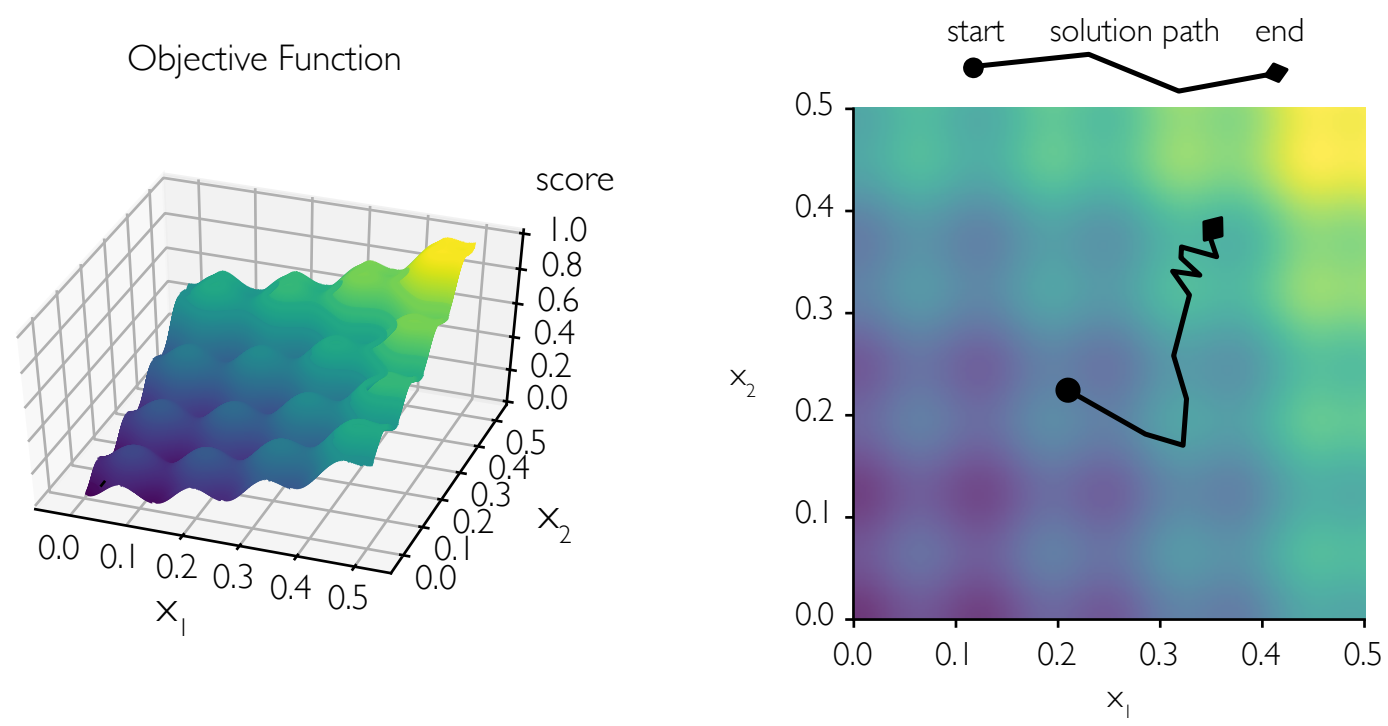

Figure 2.2. Simulated annealing involves agents exploring a solution space (right) in order to maximize a defined objective function (left)

\subsubsection{Simulating Cognitive Style}

The agents' exploration of the solution space depends on their overall KAI score, as well as the three sub-factor scores of Sufficiency of Originality (SO), Efficiency (E), and Rule/Group Conformity (RG). People with higher KAI scores tend to make larger changes to a design in search of a different solution, while people with lower KAI scores tend to make smaller changes to refine an existing solution. In the simulation, the distance an agent moves in the solution space on one turn (iteration) is positively correlated with the total KAI score. Figure 2.3 illustrates the differences in step size for agents of adaptive, mid-range, and innovative styles in a 2-dimensional problem. When searching for new solutions, a more adaptive agent moves in smaller, incremental steps, tweaking the solution with marginal adjustments. In contrast, a more innovative agent moves in larger leaps, often generating ideas distant and very distinct from their current solution.

Each agent has an individual speed parameter with an initial value dependent on their total KAI score. The speed decays geometrically throughout the course of the simulation. The distance, $D$, from the current solution to the next solution an agent generates is drawn from a chi $(\chi)$ distribution and scaled by the agent's current speed, $s$ :

$$
D=s \cdot \chi
$$

where $\chi$ is a random variable characterized by the chi probability density function with $k=1.9$ degrees of freedom. The minimum travel distance in one step is set to one ten-thousandth of the entire space, and there is no upper bound. 


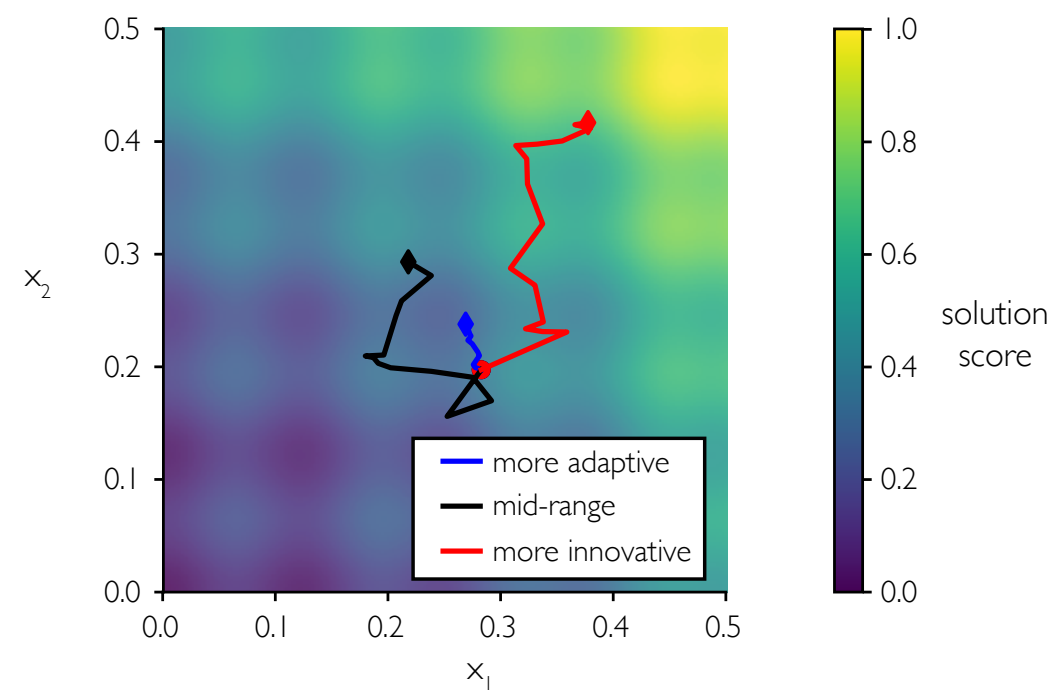

Figure 2.3. Agents with more adaptive style (lower KAI score) move in smaller steps on each iteration, while agents with more innovative style (higher KAI score) take larger steps in the solution space.

The agent's start speed, $s_{0}$, is calculated as

$$
s_{0}=\mu_{s}+K A I^{*} \cdot \sigma_{s}
$$

where $K A I^{*}$ is the standardized KAI score (re-scaled for a mean of 0 and a standard deviation of 1), $\mu_{s}$ is the average starting speed for all agents, and $\sigma_{s}$ is the standard deviation of starting speed across all agents.

\subsubsection{Efficiency $(\mathrm{E})$}

Efficiency describes an individual's preference for structure in their working methods, which range from applying highly detailed and incremental improvements to a solution (more adaptive) to dramatically or tangentially altering a solution, with less regard for detail and quality (more innovative). In KABOOM, an agent's starting temperature is correlated with the E sub-score. Temperature determines the probability of an agent accepting a candidate solution which does not improve the objective function relative to their own current solution. Temperature always decreases geometrically over the course of the simulation, but both the start temperature $T_{0}$ and the cooling rate are a function of E. An agent with a higher (more innovative) E sub-score starts with a higher temperature, and thus a higher probability of accepting solutions that do not improve the objective function. This can cause them to leave good solutions behind and miss quality nearby solutions, but can also allow them to escape local minima to find better solutions in a rough optimization topology. Agents with higher Efficiency scores also cool down slower, meaning they might never reach a very low temperature, or refine a single local solution. In contrast, an agent with a lower (more adaptive) E sub-score starts with a lower temperature, 


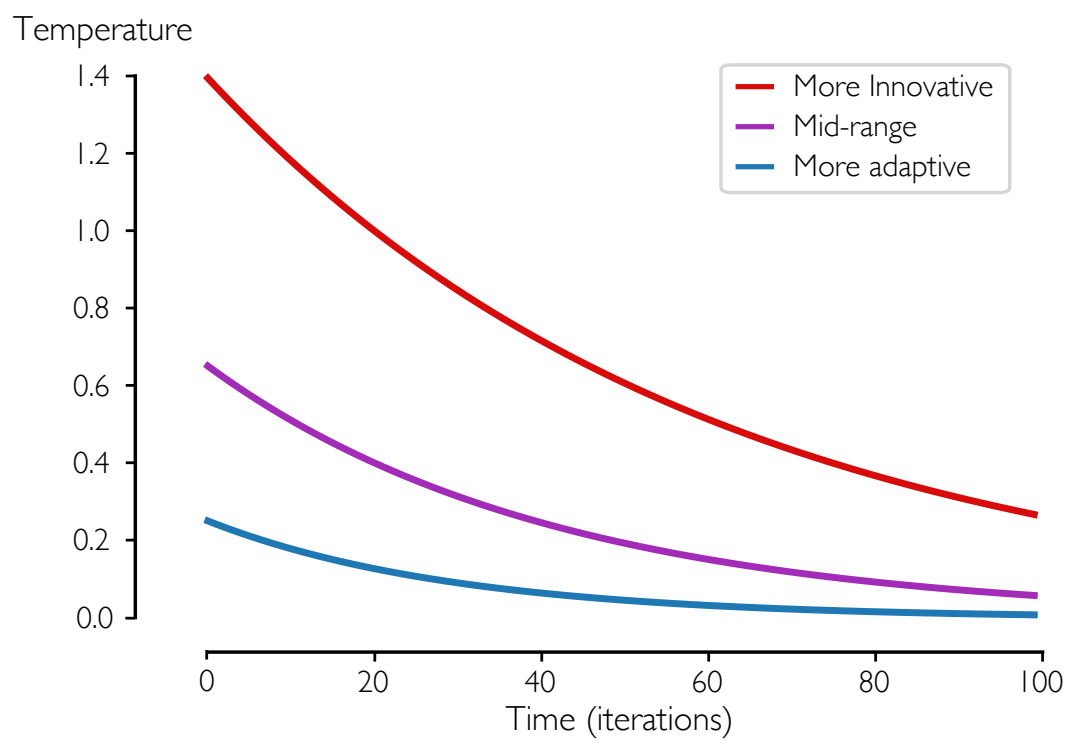

Figure 2.4. Cooling schedules for agents with different Efficiency sub-scores

and thus a lower probability of accepting solutions that do not improve the objective function. This can cause them to quickly achieve locally optimal results, but can also lead them to become stuck in local minima. The resulting behavior of the agents overall is that the more adaptive agents choose a solution early on and polish it to perfection, while the more innovative agents spend most of their time exploring very diverse solutions without refining them. The agent's start temperature $T_{0}$ is calculated as

$$
T_{0}=\mu_{T}+E^{*} \cdot \sigma_{T}
$$

where $E^{*}$ is the standardized Efficiency sub-score (re-scaled for a mean of 0 and a standard deviation of 1$), \mu_{T}$ is the average temperature for all agents, and $\sigma_{T}$ is the standard deviation of temperature across all agents.

The agent's ratio of start temperature $T_{0}$ to final temperature $T_{f}$ is

$$
\frac{T_{0}}{T_{f}}=\frac{1}{e^{2-E^{*}}}
$$

bounded by $10^{-10}$ and 1 . The geometric decay ratio for each time step is calculated based on start temperature, start-to-end ratio, and the number of steps in the simulation. Figure 2.4 shows the temperature over the course of a simulation for agents with more innovative (high E), mid-range (mid-range E), and more adaptive (low E) Efficiency sub-scores. The agent's speed decays with the same ratio as the temperature.

In simulated annealing, the probability that an agent will accept or reject a solution that is inferior to its current solution depends on its temperature and the difference in solution quality 


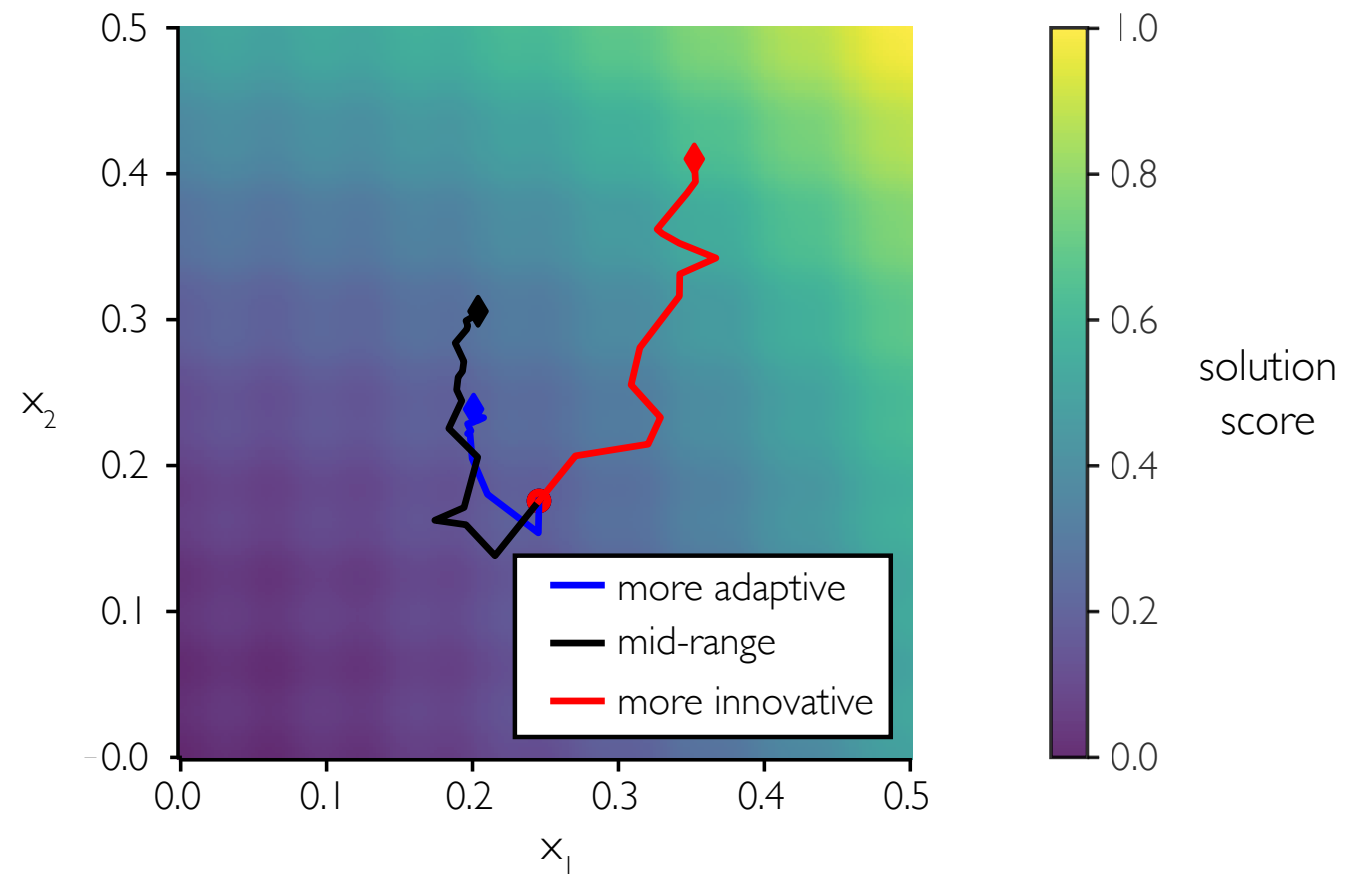

Figure 2.5. The Efficiency sub-factor of style impacts agents risk-aversion (adaptive) or risk-affinity (innovative) of agents in solution space exploration

according to Equation 2.5 [54]. At high temperatures, an agent will accept solutions regardless of quality, but at low temperatures, agents only accept solutions that improve the objective function, as shown below:

$$
P_{\text {accept }}=\exp \left(\frac{f\left(\vec{x}_{n}\right)-f(\vec{x})}{k_{B} T}\right), \quad f\left(\vec{x}_{n}\right)<f(\vec{x})
$$

where $P_{\text {accept }}$ is the probability that an agent with current solution $\vec{x}$ accepts a new solution $\vec{x}_{n}$ that does not improve the objective function $f$, as a function of current temperature $T$ and a constant $k_{B}$ which we can absorb into the calculation of temperature.

Figure 2.5 illustrates the effect of the Efficiency style sub-factor on three agents' exploration of the solution space. Compared to the mid-range agent (black), the more adaptive agent (blue) refines an early solution, while the more innovative agent (red) continuously explores without refining a solution.

\subsubsection{Sufficiency of Originality (SO)}

An agent's desire to retain and modify known solutions or, conversely, to explore more unfamiliar ideas is related to the Sufficiency of Originality sub-score of KAI. When agents evaluate a candidate solution in KABOOM, more adaptive agents are more likely to accept a candidate solution that directs them towards their previous solutions and less likely to accept a solution that leads 


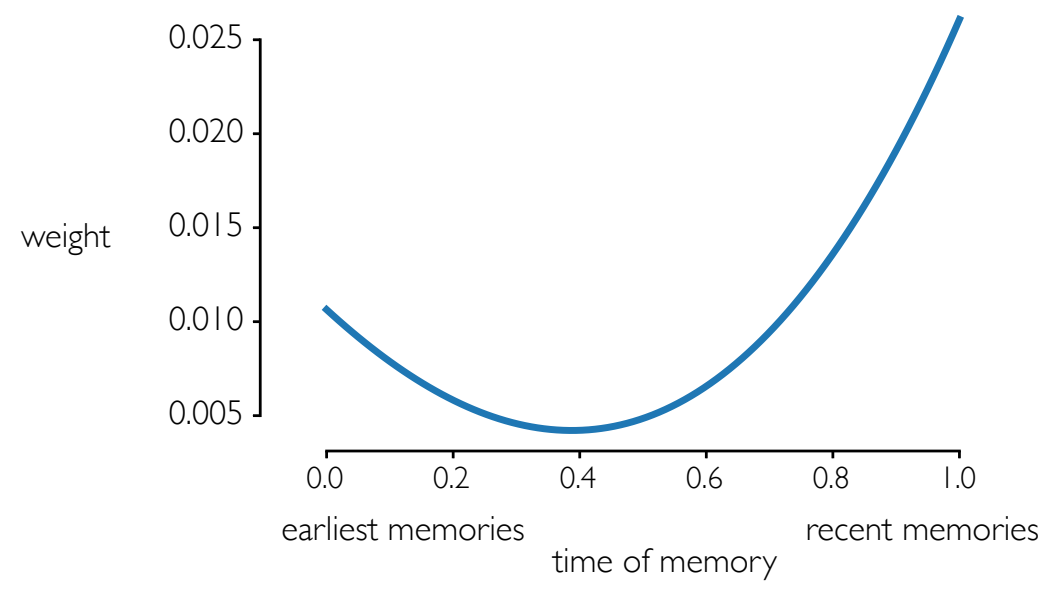

Figure 2.6. In calculating the perceived memory location, an agent's past memories are weighted based on the serial position effect reflected in this curve: the most recent memories and earliest memories are recalled more easily than intermediate memories.

them away from previous solutions. On the other hand, more innovative agents prefer to choose ideas that move them further from ideas they have explored in the past and to reject ideas that lead them towards their previous solutions. An agent's perception of their previous solutions is represented by a single point in the solution space, which is a weighted mean (centroid) of all of their previous positions or "memories". The weights on an agent's memories are strongest for the most recent memories, weakest for middle memories, and intermediate strength for the earliest memories (Figure 2.6). This reflects the serial position effect [57], which describes how people tend to remember early memories (primacy effect) and recent memories (recency effect) more readily than intermediate memories.

The direction of the weighted memory position from the current position is a weighted average of the directions to all previous solutions the agent has visited. We call $\vec{v}_{m e m}$ the perceived memory direction. The weights are given by the primacy-recency bias function $Q$,

$$
\vec{v}_{m e m}=\sum_{n=1}^{N}\left(\vec{x}-M_{n}\right) * Q(n)
$$

where the memory $\mathrm{M}$ is a list of $\mathrm{N}$ previous solutions (each memory being a vector of the same shape as the current solution $\vec{x}$ ). The primacy-recency bias function for memory $\mathrm{n}$ of $\mathrm{N}$ total memories is:

$$
Q(n)=n^{3}-0.4(N-n)^{3}
$$

which gives a U-shaped curve with stronger biases for the earliest and most recent memories, respectively, than for other memories (Figure 2.6). The weights are normalized so that they sum to 1.

The direction of the candidate solution from the current solution is the vector difference between the candidate solution and the current solution, $\vec{v}_{n}=\vec{x}_{n}-\vec{x}$. The dot product of these 
two directions indicates whether the new solution is in the direction of the perceived memory or away from the perceived memory. We call the normalized dot product the originality $\Omega$ :

$$
\Omega=\left\|\vec{v}_{m e m} \cdot \vec{v}_{n}\right\|
$$

Then, the Sufficiency of Originality preference $P_{S O}$ is:

$$
P_{S O}=\Omega \cdot S O^{*} \cdot W_{S O}
$$

where $S O^{*}$ is the standardized SO score (re-scaled for a mean of 0 and a standard deviation of 1 ), and $W_{S O}$ is a global scaling constant that determines the strength of the SO preference. For this paper we set $W_{S O}$ to 2 , which creates a range of behaviors for agents across the KAI cognitive style spectrum. When an agent evaluates the quality of a candidate solution, the SO preference $P_{S O}$ and RG preference $P_{R G}$ (explained below) influence the perceived solution quality: the preferences are added to the true value of the objective function for the candidate solution. In other words, the perceived solution quality $f_{P}$ of a candidate solution $\vec{x}_{n}$ is related to the true solution quality $f\left(\vec{x}_{n}\right)$ by

$$
f_{P}(\vec{x})=f(\vec{x})+P_{S O}+P_{R G}
$$

Figure 2.7 illustrates the effect of the SO cognitive style sub-factor on three agents' exploration in a 2-dimensional solution space. The black line shows the solution path of an agent with mid-range cognitive style. The red line shows the path of a more innovative agent in the same space, with only the Sufficiency of Originality preference active (and otherwise identical to the mid-range agent). The innovative SO style gives the agent a preference for solutions that move it away from its previous solutions, leading the agent to explore further into the corner of the solution space. On the other hand, the blue line shows the more adaptive agent's preference for solutions that are close to its previous solutions, leading to a dense packing of solutions near its original starting point.

\subsubsection{Rule/Group Conformity (RG)}

The third cognitive style sub-score, rule/group conformity, describes an individual's preference for managing structure in both impersonal and personal contexts. More adaptive individuals prefer to leverage existing impersonal structures, such as rules, guidelines, and precedents, while more innovative individuals are more likely to bend or violate these structures. A similar pattern of behavior emerges for personal structures, such as groups or teams. A person with an adaptive rule/group conformity style tends to cohere with the group and seek group consensus, while a more innovative person tends to diverge from and may even disrupt the group.

In order to capture one aspect of the rule/group conformity sub-factor, the present model focuses on conformity to personal structures (i.e. individuals' desire to converge or diverge from group consensus). Future work could develop other aspects of rule/group conformity, for instance, 


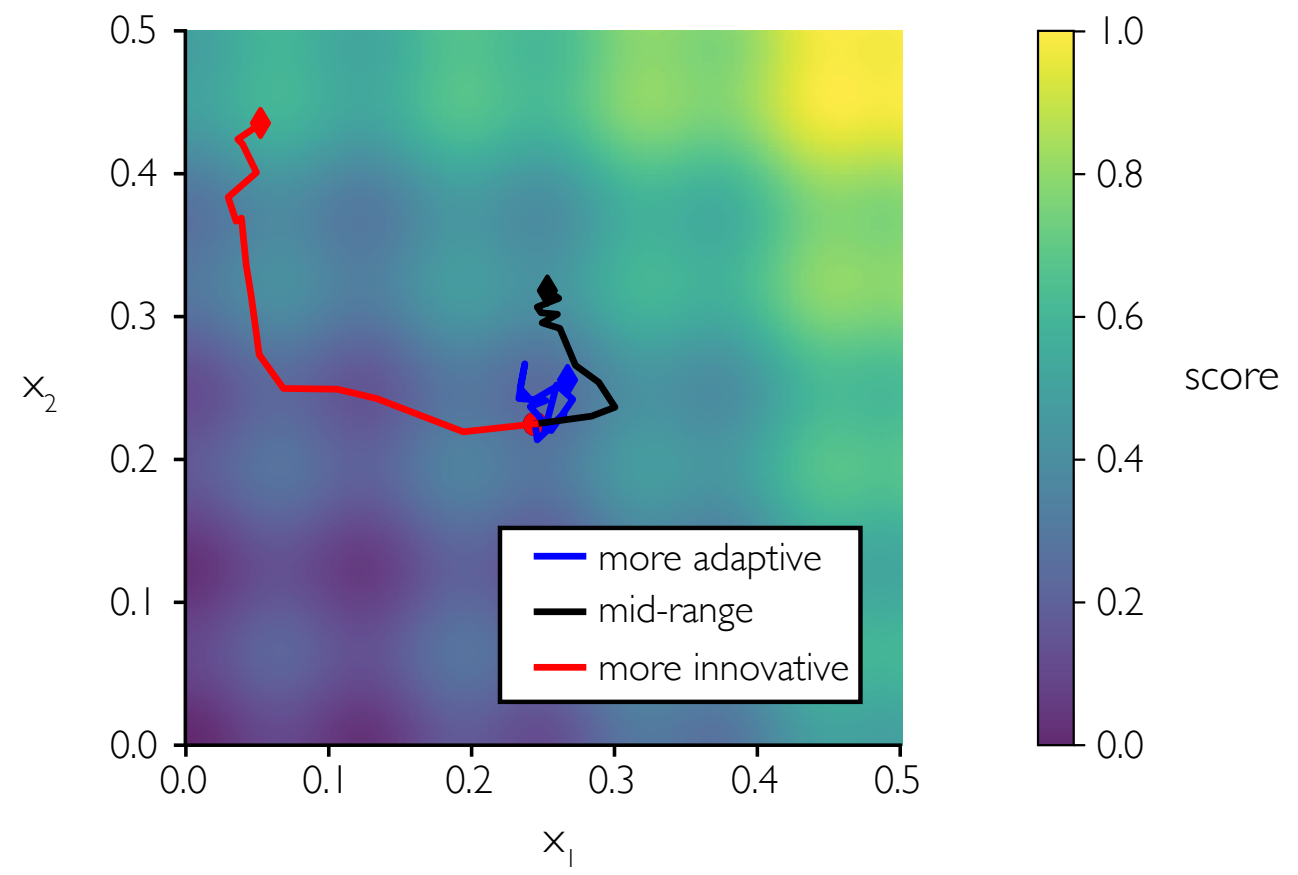

Figure 2.7. The Sufficiency of Originality Effect sub-factor of style influences an agent's preference for familiar (adaptive) or novel (innovative) ideas during solution space exploration.

by modifying the agents' adherence to optimization constraints. In KABOOM, the more adaptive agents are more likely to move towards solutions that bring them closer to the team's average position, thus encouraging group cohesion. More innovative agents, on the other hand, have a preference for solutions that move them away from the mean position of the group.

In KABOOM, this aspect of rule/group conformity is implemented in a similar way to Sufficiency of Originality by replacing an agent's memories with the current solutions. In other words, while SO makes agents favor solutions towards (more adaptive) or away from (more innovative) their own previous solutions, $R G$ gives agents a preference towards (more adaptive) or away from (more innovative) the current solutions of their teammates (Figure 2.8).

A "team position" $\vec{x}_{t e a m}$ is represented by the centroid of the members of the team:

$$
\vec{x}_{\text {team }}=\frac{1}{N} \sum_{n=1}^{N} T_{n}
$$

where $\mathrm{T}$ is the team of $\mathrm{N}$ agents, and $T_{n}$ selects the current solution of each agent on the team. As before, the direction of the candidate solution from the current solution is the vector difference, $\vec{v}_{n}=\vec{x}_{n}-\vec{x}$. Likewise, the direction of the team position from the current position is $\vec{v}_{\text {team }}=\vec{x}_{\text {team }}-\vec{x}$.

The dot product of these two vectors indicates whether the new solution is in the direction 

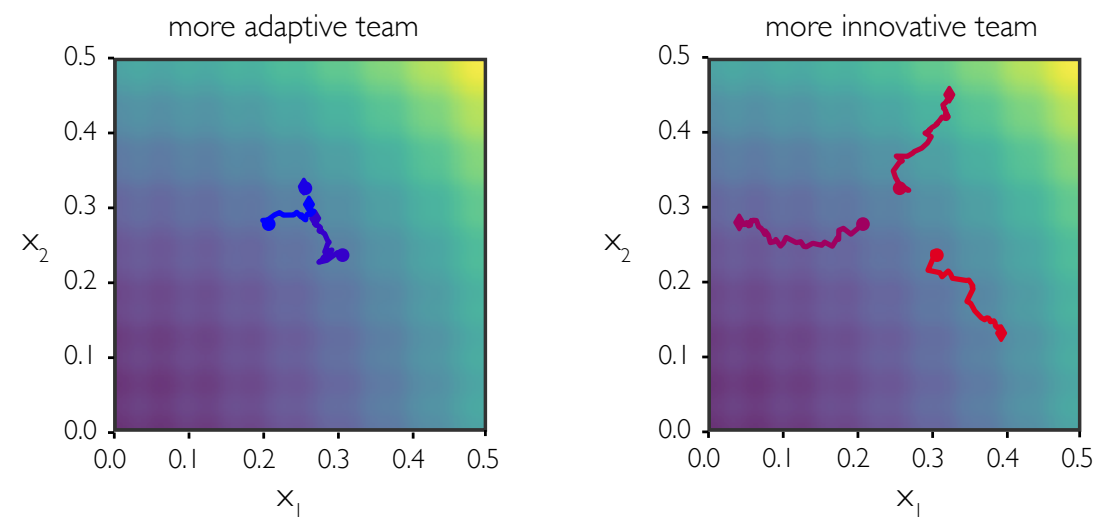

Figure 2.8. The Rule Group conformity sub-factor of style is demonstrated for homogeneous teams of adaptive (left) style and innovative (right) style. The adaptive team converges while the innovative team diverges.

of the team (positive dot product) or away from the team (negative dot product). We call the normalized dot product the conformity $C$ :

$$
C=\left\|\vec{v}_{\text {team }} \cdot \vec{v}_{n}\right\|
$$

Then, the group conformity preference $P_{R G}$ is:

$$
P_{R G}=C \cdot R G^{*} \cdot W_{R G}
$$

where $R G *$ is the standardized Rule/Group conformity sub-score (re-scaled for a mean of 0 and a standard deviation of 1 ), and $W_{R G}$ is a global constant used to change the strength of the RG preference, which is set to 2 to create a range of behaviors.

Figure 2.8 illustrates the effect of the Rule/Group conformity cognitive style sub-factor for adaptive and innovative teams, respectively, of three agents each. Agents with a more adaptive RG style prefer solutions that bring them towards their team, resulting in team convergence (left), while more innovative agents seek solutions away from their team, leading to team divergence (right).

\subsubsection{Interactions and Communication}

KABOOM contains two types of agent interactions in which agents on a team share their solutions with each other, pairwise communication and team meetings.

\subsubsection{Pairwise Communication}

In models of team problem solving, communication can be modeled using organically timed pairwise interactions between team members [33]. In KABOOM, agents collaborate and share 
solutions by sharing their current position in the solution space with each other. On a given turn, an agent can either explore a new solution individually or communicate with another agent to share solutions. The probability of an agent deciding to communicate in a pairwise interaction on a given turn is set by a model parameter $c$, which can be constant in time or change over time. On each turn, agents on the same sub-team who decide to collaborate are paired randomly. If there is an odd number of agents who wish to collaborate, the unpaired agent explores individually for that turn.

After sharing their current solutions, each agent evaluates the solution shared with them and chooses whether they want to move to that solution. As with evaluating a potential solution in individual exploration, the probability of accepting a solution that does not improve the objective function in favor of the current one is a stochastic function of the agent's temperature (Equation 2.5). When the temperature is zero, there is zero probability of choosing a solution that does not improve the objective function.

Research shows that when the difference in two people's KAI total scores is greater than 20 points, communicating ideas with each other tends to become increasingly difficult [5]. Agent collaboration in KABOOM reflects this increasing difficulty in communication due to this cognitive gap in style. In the model, communication between two agents always has some probability of failing; this likelihood is positively correlated with the difference between the agents' KAI total scores (i.e., their cognitive style gap). This is implemented by requiring a uniformly distributed random variable to be less than the difference in two agents' KAI total scores for successful communication. If communication is not successful, no information is shared between the agents, essentially resulting in a wasted iteration.

The probability of successful collaboration is:

$$
P=1-(\Delta K A I-10) / 170
$$

where $\triangle K A I$ is the difference between the two agents' total KAI scores. This results in the communication success curve shown in Figure 2.9. Agents with KAI total score differences of 10 points or less have a $100 \%$ success rate, which drops linearly beyond the 10-point just-noticeabledifference established in prior research [5]. Communication across extremely large gaps of 100 points is modeled as only being successful $50 \%$ of the time (the observed KAI range is 109 points [23]).

\subsubsection{Team Meetings}

In addition to pair-wise communication, agent teams have regularly scheduled meetings in which all team members converge to a single solution. First, the team creates an aggregate solution from the specialized sub-teams: each sub-team finds the best solution from any of its agents, then contributes only its dimensions to the aggregate team solution. For example, if there is a 2-dimensional solution space where sub-team 1 controls $x_{1}$ and sub-team 2 controls $x_{2}$, the aggregate solution is $<x_{1}$ from sub-team $1, x_{2}$ from sub-team $2>$. Finally, all agents accept the 


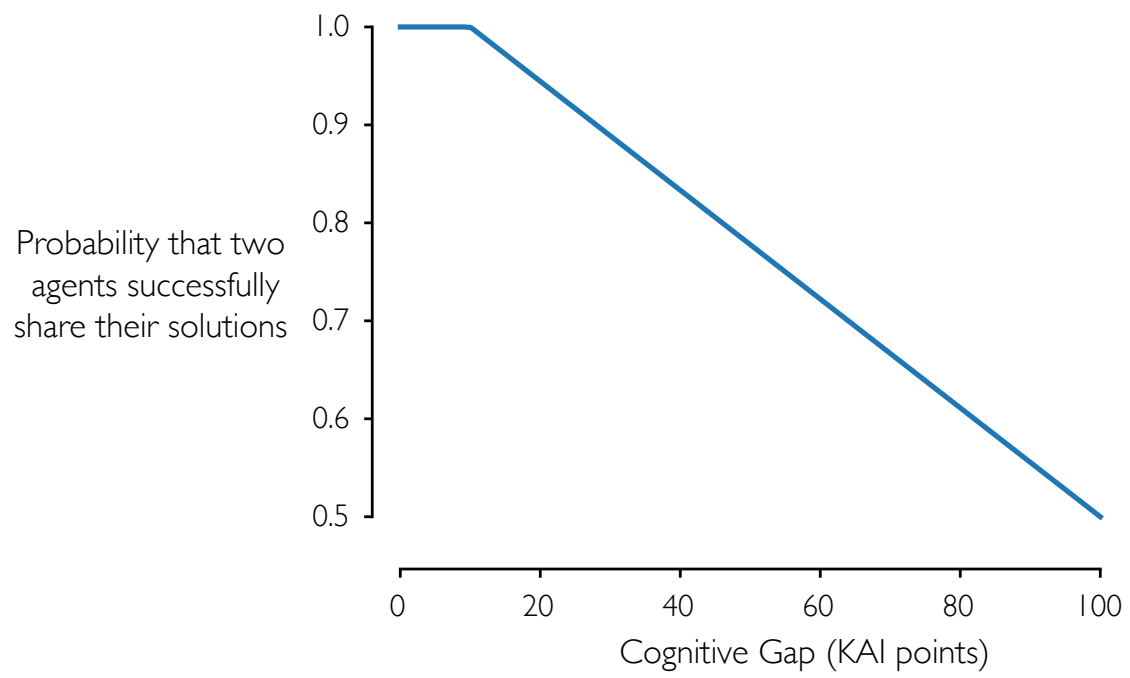

Figure 2.9. The probability of successful communication decreases as the difference in the two agents' KAI scores (cognitive gap) increases.

new aggregate position, regardless of the quality of the new aggregate solution.

\subsubsection{Creating a virtual population with KAI}

We create a virtual population of individuals such that the distributions and correlations of KAI scores and sub-scores (SO, E, and RG) reflect those of the general population. A Python script generates KAI scores and sub-scores for 10,000 virtual individuals from a multivariate normal distribution. The mean, standard deviation, and correlations of KAI, SO, E, and RG are based on a dataset of 597 individuals' scores and sub-scores gathered in previous research [20]. The sub-scores are all imperfectly correlated $\left(0.4<R^{2}<0.8\right)$ with the total KAI score. Because of this, even agents with the same KAI score will have a range of sub-scores for SO, E, and RG, meaning that any two agents with the same total KAI score are unlikely to be precisely identical in their style (as with humans). KABOOM draws from this virtual population to create agents with associated KAI scores. When the model requests an agent with a specific total KAI score (for instance, 95), it receives a randomly selected member from all individuals in the virtual population having the requested KAI score. Thus, requesting two agents with KAI total scores of 95 will return two different agents, each having total scores of 95 but (likely) having different sub-scores for SO, E, and RG.

\subsubsection{Selecting agents for a team}

When forming a team of agents, KABOOM selects individuals from the virtual population according to a team composition strategy. This paper uses three team composition strategies:

1. Organic Composition: team members are drawn randomly from a virtual population that is statistically representative of the true distribution of KAI scores; 
2. Homogeneous Composition: all team members have the same KAI total score (but may have different sub-scores);

3. Linearly Distributed Heterogeneous Composition: the team is composed with a given mean and range of KAI total scores. The team will be composed of agents linearly distributed across the range and centered on the mean. For example, a linearly distributed 5-person team with mean KAI of 100 and a range of 40 will be composed of five agents with KAI scores 80, 90, 100, 110, and 120 .

When teams are divided into specialized sub-teams, each sub-team (rather than the full team) is selected to have a linearly distributed composition with the given mean and range. For example, given a team of 4 with 2 sub-teams of 2, and requiring a KAI mean of 100 and range of 40, each sub-team will be composed of two agents with KAI scores of 80 and 120.

When plots in this thesis compare agents or homogeneous teams of three styles, the KAI scores are 55 (adaptive), 95 (midrange), and 135 (innovative). These represent the 1.5th, 50th, and 98.5th percentiles of the population; therefore, $\mathrm{KAI}=55$ and $\mathrm{KAI}=135$ represent extremes of cognitive style behavior that are unlikely (but not impossible) to be observed in real life. These are intended to demonstrate the range of possible behaviors across the KAI spectrum. Organic composition gives more realistic team compositions.

\subsubsection{Model Parameters}

Parameters in the model are generally tuned to reflect observed human behavior. Except where noted, the parameters were held constant at the values listed in Table 2.1. The objective function parameters are introduced in Section 2.2. An appendix contains a table listing all parameter values for each figure that contains results from the abstract problem.

\subsubsection{Evaluating Performance}

At the end of the simulation, each team's performance is the solution quality of the best solution any agent has had at any time during the simulation. Because the KABOOM model is stochastic, it repeats the same experiment 16 times (except where otherwise noted) before changing any

parameters. However, due to the team selection methods described above, each iteration uses a new team that is not composed of exactly the same agents. In this paper, plots of results show the mean and standard deviation of team performance with a dot and vertical error bar (shown in blue unless there are multiple series on one plot). In some plots, separate team scores from each repetition are shown as gray dots. Linear and quadratic best-fit equations are displayed as red curves. 
Table 2.1. List of model parameters and their default values (Objective Function parameters refer to the abstract objective function presented in Section Section 2.2

\begin{tabular}{lccl} 
Parameter & Symbol & Value & Description \\
\hline General Parameters & & & \\
\hline Iterations & & 300 & num. iterations in one simulation \\
Team size & & 6 & num. agents on the team \\
Number of sub-teams & & 2 & num. specialized teams \\
Agents per sub-team & 3 & num. agents on each sub-team \\
Average starting temperature & $\mu_{T}$ & 1 & \\
Standard deviation of temperature & $\sigma_{T}$ & 0.8 & \\
Average starting speed & $\mu_{s}$ & 0.01 & \\
Standard deviation of speed & $\sigma_{s}$ & 0.007 & \\
& & & \\
Objective Function Parameters & & & \\
\hline Oscillation amplitude & $\alpha$ & 0.1 & \\
Scaling parameter & $\beta$ & 1 & \\
Feasible solution space & & {$[-1,1]$} & each dimension bounded by range \\
& & & \\
Communication Parameters & & & \\
\hline Communication frequency & $c$ & 0.2 & prob. agent communicates on turn \\
Meeting interval & & 50 & num. iterations between meetings
\end{tabular}

\subsection{Abstract Objective Function}

KABOOM can model any problem that can be expressed using an objective function of a finite number of continuous variables. Chapter 3 uses an abstract objective function composed of sinusoids and parabolas that is not tied to a physical problem, because its characteristics can be continuously tuned using a set of parameters. This allows us to compare performance of different cognitive styles while continuously varying parameters of the objective function. Chapter 4 uses two contextualized problems in order to relate the model results to real-world problems: a relatively simple I-beam design problem and a relatively complicated race car design problem. The implementation of each objective function is described below.

\subsubsection{Objective Functions}

The design problem is represented by a scalar objective function $f(\vec{x})$ of $n$ dimensions (variables). A solution defines the value of all variables, meaning a solution is a vector $\vec{x}$ in the $\mathrm{n}$-dimensional space. The quality of the solution is defined as the objective function's value at that point, $f(\vec{x})$. The goal is to maximize solution quality. Real world problems can sometimes be formulated as analytic objective functions with several variables and constraints, as in Zurita et al. [1]. However, this paper implements a more abstract mathematical objective function, so that it can be tuned and scaled in predictable ways. The objective function used throughout this paper is a summation of a quadratic function and a sinusoidal function in the form: 

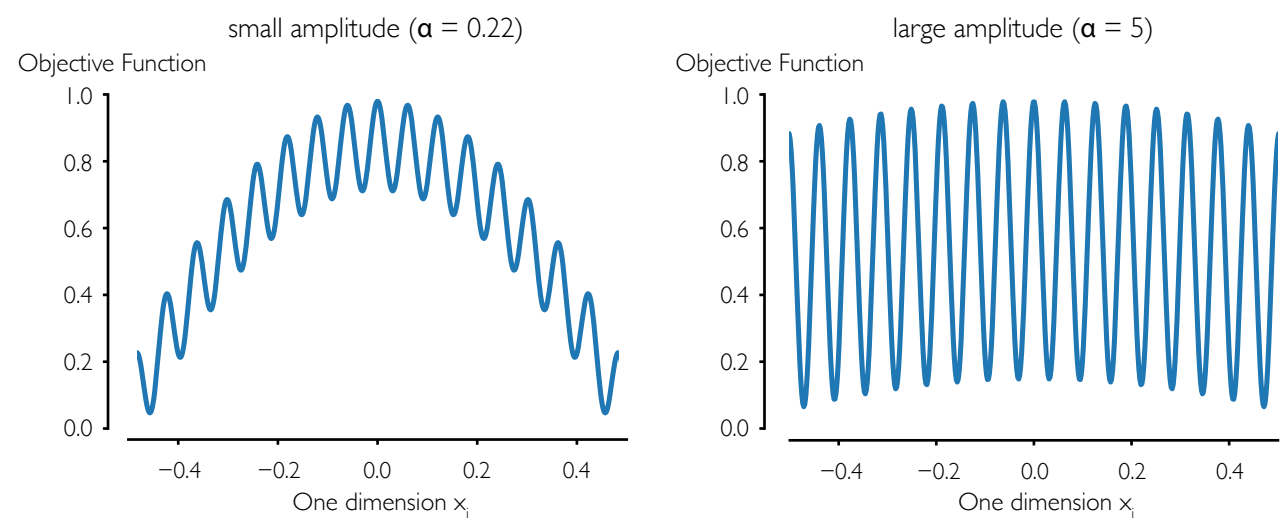

Figure 2.10. The objective function is a composite of a sinusoid and a parabola. The oscillation amplitude $\alpha$ of the objective function is varied from 0.22 to 5 . Global exploration of the space is important when $\alpha$ is small, but local exploitation is sufficient when $\alpha$ is large. The vertical axis shows normalized solution quality.

$$
f(\vec{x})=-\sum_{i=1}^{n} \alpha \sin \left(\frac{\omega \vec{x}_{i}}{\beta}\right)+C\left(\frac{\vec{x}_{i}}{\beta}\right)^{2} \quad \text { for }-0.5 \leq x_{i} \leq 0.5
$$

Figure 2.10 shows the objective function in one dimension for two values of $\alpha$. The objective function can be defined in any number of dimensions, $n$, and is symmetric in all dimensions. The oscillation frequency, $\omega$, and quadratic coefficient, $C$, are held constant. The space is bounded by $[-1,1]$ in all dimensions: solutions outside of this cube are infeasible. In order to focus on the relative performance of teams for a given problem, all performance axes for figures in this paper are normalized to the range of 0 to 1.

This function is varied in two ways: (1) by scaling the independent variables in all dimensions using the scaling parameter, $\beta$, and; (2) by scaling the amplitude of the sinusoid, $\alpha$. The first parameter affects the size of the search space, while the second parameter affects the amplitude of the sinusoid. Changing these two characteristic values creates a variety of different problems that may favor different cognitive styles.

\subsubsection{Contextualized Race Car Design Problem}

The first contextualized problem is the design of an SAE race car, which was formulated by Zurita et al. [1] to evaluate multi-agent coordination on a decomposed problem. We use the same problem with minor modifications in order to evaluate team performance using KABOOM. In this section, we only provide general information on the implementation of the problem for $\mathrm{KABOOM}$; the reader is referred to the original publications for the remaining details.

The race car design problem parameterizes the design of a racing vehicle into 56 variables, which are divided among eleven sub-systems, such as brakes, engine, and front suspension (see Figure 2.11). Agents belong to one of eleven sub-teams, corresponding to each of the subsystems. 


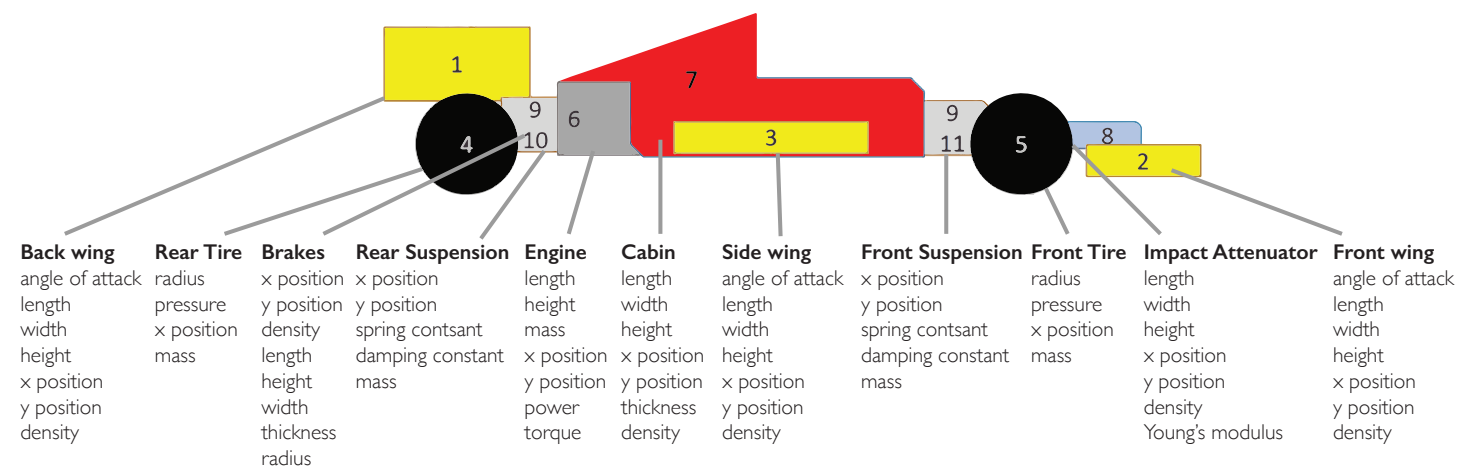

Figure 2.11. The variables in the car design problem are distributed among specialized teams. Figure adapted from Zurita et al. [1] Figure 3 and Table 1.

Therefore, each agent explores the solution space only in the sub-space of their team's dimensions (i.e., an agent on the brakes team only modifies the brakes variables). The performance of the team is evaluated based on a global objective function, which is composed of eight sub-objectives, each describing an appropriate metric, such as acceleration, breaking distance, or turning radius. After standardizing the performance on each sub-objective (rescaling to a mean of zero and standard deviation of one), the total objective score is calculated as a weighted sum of the subobjective scores. This paper uses Weight Scenario 2 of Zurita's three weighting schemes ([1] Table 2) for the weighted sum of sub-objectives to form a scalar objective function. See Zurita et al. [1] for a full description of the dimensions, sub-objectives, and constraints of the race car design problem.

In order to maintain a continuous solution space for the KABOOM model, discrete-valued variables are mapped onto a continuous space by selecting one driving dimension for each discrete variable. When the objective function is evaluated, the variable is mapped back onto the nearest feasible discrete value. For example, the choice of material for the race car cabin is a discretevalued variable and is chosen from a table that lists density and modulus of elasticity for several materials. We select density as the driving variable and represent material density as a continuous variable in the solution space. When an agent evaluates the objective function, it chooses the material with density nearest to the current continuous value. Besides material choice, the only discrete-valued variables are related to the engine (driving dimension is power) and the wheels (driving dimension is radius).

Additionally, the solution space is normalized to a unit cube, so that agents can explore each dimension equally. The minimum and maximum values of each parameter are used to rescale the feasible space to the interval $[0,1]$. Thus, feasible solutions to the problem are vectors of length 56 with values in $[0,1]$. This normalized vector is re-scaled into real-world units when evaluating the objective function (which is implemented in SI units). 


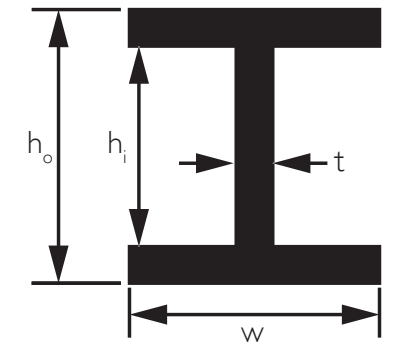

(A)

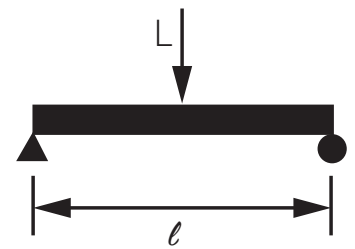

(B)

Figure 2.12. Design dimensions of (A) a beam cross section and (B) beam loading

\subsubsection{Contextualized Beam Design Problem}

This section describes an objective function for designing a wide-flange beam in order to compare the design of a complex system (the race car) with a simpler design problem (the beam). The beam design problem has four design variables related to beam geometry (Fig $2.12 \mathrm{~A}$ ) and an objective of minimizing the maximum stress and displacement for a fixed load. The beam is simply supported, with fixed length and a point load at the center (Fig 2.12 B). The equations used here can be found in Gere's Mechanics of Materials [58].

The objective function is a weighted sum of maximum stress and displacement:

$$
f(x)=\delta^{*}(x) \cdot 10^{3}+\sigma^{*}(x) \cdot 10^{-6}
$$

where $\delta^{*}(x)$ is the maximum displacement of the beam

$$
\delta^{*}(x)=\frac{-L l^{3}}{48 E I}
$$

and where $L$ is the center point load, $l$ is the length of the beam, $E$ is the modulus of elasticity, and $I$ is the moment of inertia calculated with

$$
I=\frac{1}{12}\left[w\left(h_{i}^{3}-h_{o}^{3}\right)+t\left(h_{i}^{3}\right)\right]
$$

Because the extreme fibers of the beam are located a distance $h_{o} / 2$ from the central axis, the maximum stress $\sigma^{*}$ is given by

$$
\sigma^{*}=\frac{h_{o} L l}{8 I}
$$

where $L$ is the center point load, $l$ is the length of the beam, and $I$ is the moment of inertia. In this paper, the load $L$ is 50,000 Newtons $(\mathrm{N})$, the beam length is 5 meters $(\mathrm{m})$, and the modulus of elasticity is 200 gigapascals (GPa). The starting values for the design variables (in meters) are $h_{i}=0.23, h_{o}=0.25, w=0.1$, and $t=.02$.

All dimensions are constrained to the range $[0.007,1]$ meters, and the horizontal flange thickness (i.e. $\left.\left(h_{o}-h_{i}\right) / 2\right)$ is also constrained to this minimum thickness. Additionally, the total 
area of the cross section is constrained by a maximum value of $0.007 \mathrm{~m}^{2}$. This is equivalent to a mass constraint because of the prismatic geometry. 
CHAPTER 3

\section{Results: abstract design problems}

This chapter explores the effects of communication, specialization, and composition on team performance for the abstract design problem. In the abstract problem, which was introduced in Section 2.2, the sinusoid amplitude and the size of the solution space can be adjusted to change the characteristics of the problem. While the problem variables are normally held constant for each experiment, Section 3.1.3 varies the characteristics of the problem in order to observe the interactions of problem variables with a team's cognitive style composition.

\subsection{Independent Effects of Communication, Specialization, and Composition}

In this section, three studies examine how communication, specialization, and composition independently influence team performance for various cognitive style compositions. Communication refers to the frequency of pair-wise solution sharing and the frequency of team meetings. The first study shows that while pairwise communication can often help performance, the optimal communication rate depends strongly on the cognitive style of the team. Specialization refers to the division of the team into sub-teams which tackle pieces of the problem independently. Results from the specialization study suggest that the optimal amount of team specialization depends strongly on the cognitive styles of its members. Finally, composition refers to the range and distribution of cognitive styles on a team. By examining team performance on a set of five problems, the third study demonstrates a scenario where diversity of cognitive style on a team benefits performance. 


\subsubsection{Communication}

This section investigates the effects of communication on performance. As noted earlier, KABOOM models both pairwise communication rates and team meetings. Pairwise communication is driven by agents' desired communication frequency and has organic timing. Team meetings occur at regular intervals and result in the team converging to one unified solution.

\subsubsection{Pairwise Communication Frequency}

Our first study examined how the frequency of pairwise communication affects performance. Results of previous work suggest that there is a curvilinear (rather than linear) relationship between communication and performance, meaning that the best performance will occur at some intermediate communication rate, above which performance will decrease $[11,40,42]$. We call the agents' rate of pairwise communication the "communication policy" c. At each iteration, every agent in the simulation chooses to collaborate with another agent with probability according to the communication policy $c$ or to explore individually with probability $1-c$. Varying $c$ from zero to one represents the spectrum of strategies from individual exploration with no pairwise communication $(c=0)$ to constant communication with no exploration $(c=1)$.

Figure 3.1 shows the performance of teams with organic composition (12 agents per team, 4 sub-teams of 3) for a range of communication policies. Each gray dot represents one team's final solution quality. The blue points and lines indicate the mean and one standard deviation for the eight teams' respective performance with a given communication policy. It is clear that there is a curvilinear relationship involved, where communication improves performance up to a point and then diminishes performance. (Performance drops significantly for $c=1$, because agents never explore the solution space.) The optimal policy for this problem and team is to have a $40 \%$ to $60 \%$ chance of trying to collaborate on each turn. This result is consistent when testing with different team sizes and specializations.

The optimal communication policy changes drastically for homogeneous teams of different cognitive styles (Figure 3.2). For a homogeneous team with a shared innovative style, increasing the communication rate increases performance up to very high values $(c=0.8)$. Compared to the effect on homogeneous innovative teams, the effect of communication is much weaker for

the homogeneous adaptive teams, but there is a boost in performance at 0.2 and 0.6 . The homogeneous mid-range team performs best for $c$ around 0.4 , similar to the organic teams.

These results agree well with a human-subjects study on Adaption-Innovation style and team network structures [13]. That study found that adaptors performed best in loosely connected networks with structural holes while innovators performed best in densely connected networks.

The performance of homogeneous innovative teams with high rates of communication $(0.5<c<0.9)$ has a smaller standard deviation, as well as a higher average score. We can interpret this result by considering the balance of exploration (broad search) and exploitation (local refinement of a solution). An innovative team has little difficulty exploring the space, 


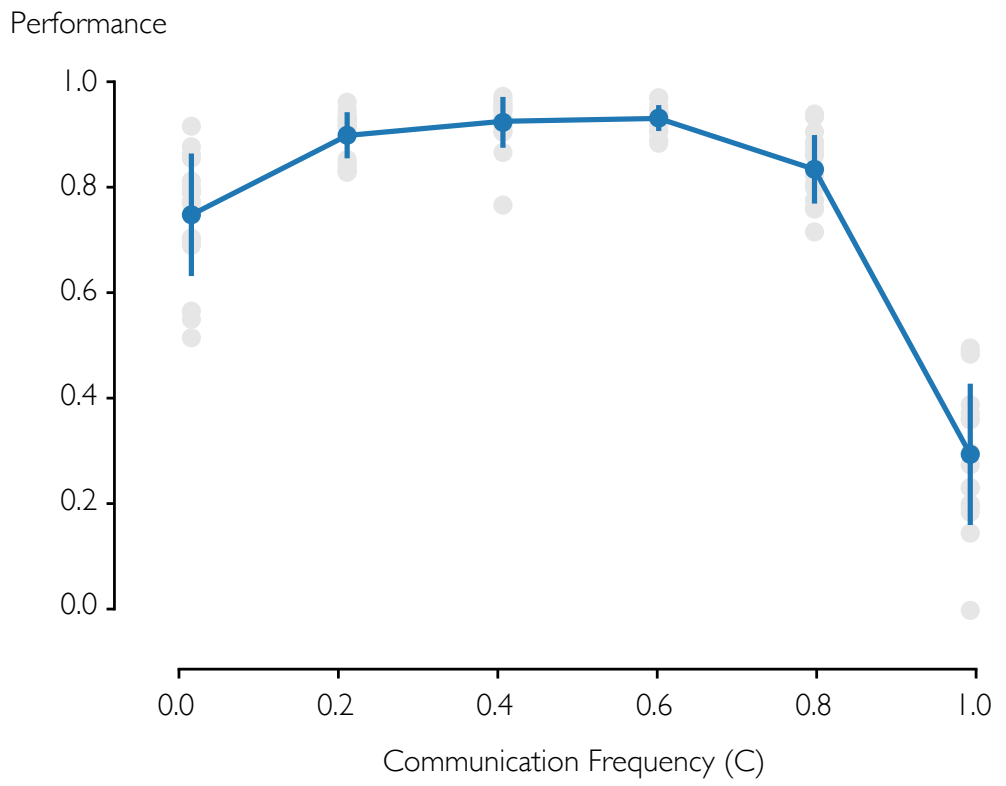

Figure 3.1. Intermediate communication rates outperform high and low communication rates for 12 agent teams of randomly selected cognitive styles specialized into 4 sub-teams (error bars indicate \pm 1 standard deviation).

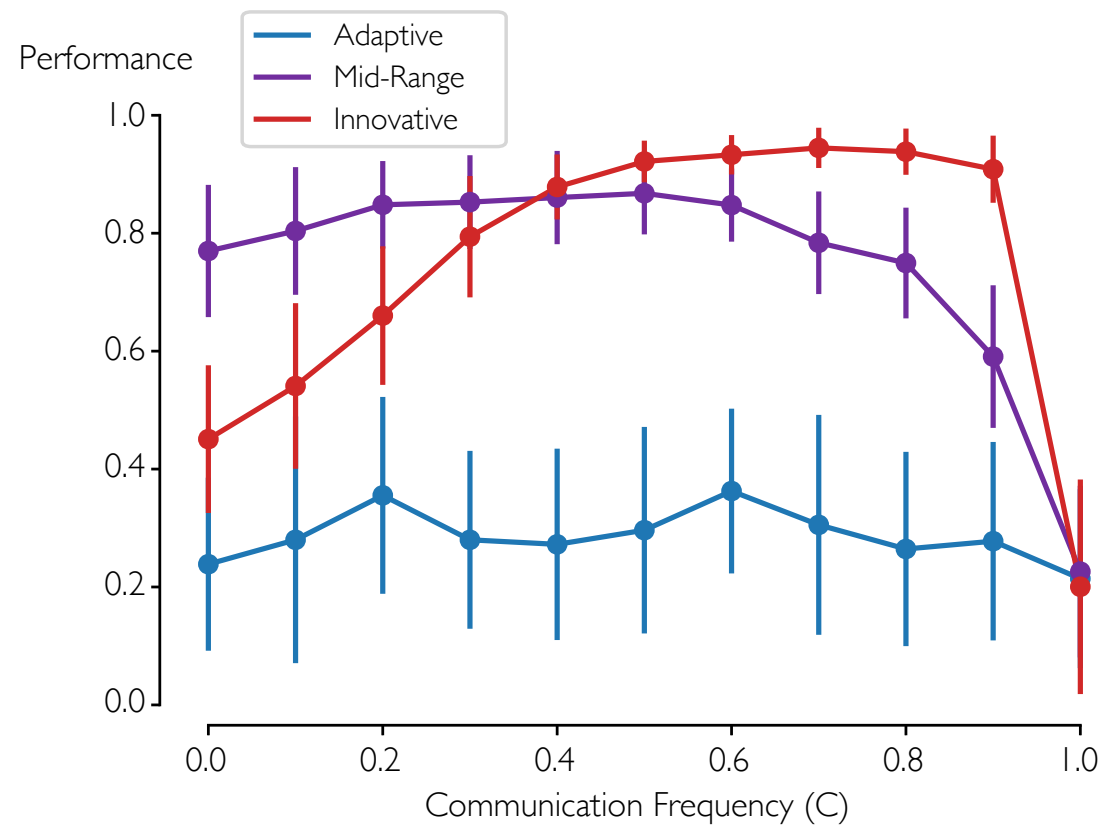

Figure 3.2. This plot shows the effect of communication frequency on team performance for homogeneous teams of adaptive, mid-range, and innovative style. Innovative teams succeed with high levels of communication, while adaptive teams show little dependence on communication (error bars indicate \pm 1 standard deviation). 
but they will not converge and refine the best solutions unless the team members interact very frequently, effectively "bringing themselves back" to the original problem-solving aim. For midrange homogeneous teams, convergence and exploitation happen more easily, but too much time spent on pair-wise communication $(c>0.6)$ inhibits thorough exploration. For more adaptive homogeneous teams, performance is less dependent on communication frequency, as more adaptive teams tend to reach consensus early and then stick to the approach they have agreed upon, which tends to focus on refining the best solutions by default. All of these patterns align well with Adaption-Innovation theory [5], as well as various validation studies with human subjects [59, 36, e.g.]. More generally, the results suggest that cognitive style plays an important role in how pairwise communication frequency affects performance.

\subsubsection{Team Meetings}

We expect team meetings to improve team performance, because they allow teams to work from the best available solutions. This hypothesis is supported by He et al. [60], which found a positive correlation between the frequency of meetings and the formation of team cognition. Figure 3.3 examines how the number of team meetings over the course of a fixed-length design period affects the team's performance for a team of six agents. For a small number of meetings, performance increases linearly with the number of meetings $\left(R^{2}=0.42, p<.001\right)$. There are 300 iterations in the simulation, so having 10 team meetings (each consuming one iteration) does not take away much of the problem-solving time. We would expect performance to saturate and eventually decrease as the number of team meetings approaches 300 .

Because a team meeting only requires one time-step in the simulation and provides a large benefit to the team, it is not surprising that more team meetings improve performance. Although KABOOM assumes team meetings are always short and successful, future work could more accurately model the cost of team meetings by accounting for the effort and time required to unify the disparate solutions of individual team members. The success of a meeting and elapsed meeting time would likely depend on both differences in team members' solutions and differences in cognitive style.

\subsubsection{Specialization}

KABOOM can rapidly simulate team performance for many different team compositions and subteam specialization configurations, giving insight into potential strategies for team organization. To demonstrate KABOOM's ability to study a wide range of scenarios, this section reports on the optimal amount of specialization in a team for different team sizes and style compositions.

Engineering teams often specialize by breaking a problem into (semi)independent pieces and assigning different parts of the problem to sub-teams [61, eg]. For example, a team designing a rocket might have sub-teams working on propulsion, stability, and aerodynamics.

In the context of this research, specialization refers to the number of sub-teams working on independent aspects of a problem. The 'independent aspects of a problem' correspond to 


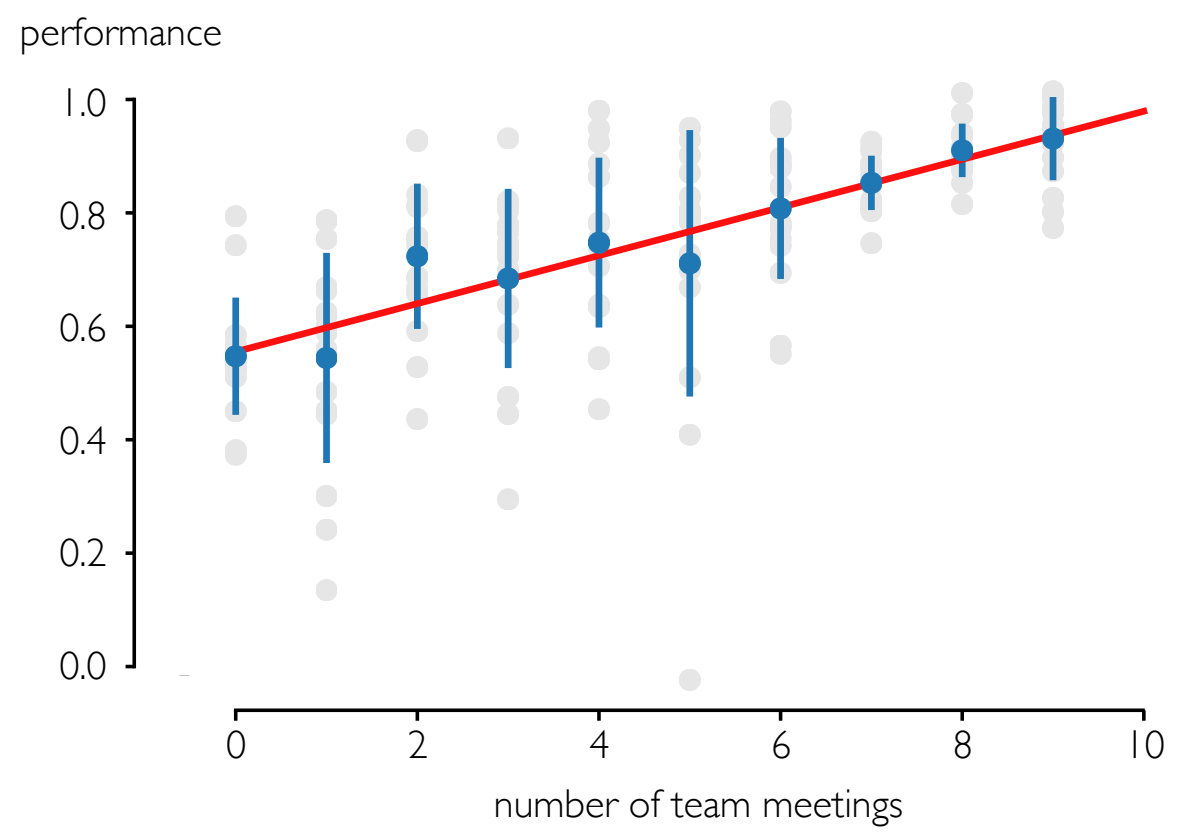

Figure 3.3. For small numbers of meetings, team performance increases linearly with the number of team meetings. During a team meeting, all agents converge to the best known solution. Error bars indicate \pm 1 standard deviation.

Table 3.1. Specialized teams decompose a problem by dividing the dimensions among sub-teams. Dimension

$\begin{array}{cccccccc}\text { Number of } & \text { Subteams } & x_{1} & x_{2} & x_{3} & x_{4} & x_{5} & x_{6} \\ \text { Subteams } & \mathrm{A} & \mathrm{A} & \mathrm{A} & \mathrm{A} & \mathrm{A} & \mathrm{A} & \mathrm{A} \\ 1 & \mathrm{~A}, \mathrm{~B} & \mathrm{~A} & \mathrm{~A} & \mathrm{~A} & \mathrm{~B} & \mathrm{~B} & \mathrm{~B} \\ 2 & \mathrm{~A}, \mathrm{~B}, \mathrm{C} & \mathrm{A} & \mathrm{A} & \mathrm{B} & \mathrm{B} & \mathrm{C} & \mathrm{C} \\ 3 & \mathrm{~A}, \mathrm{~B}, \mathrm{C}, \mathrm{D}, \mathrm{E}, \mathrm{F} & \mathrm{A} & \mathrm{B} & \mathrm{C} & \mathrm{D} & \mathrm{E} & \mathrm{F}\end{array}$

mutually exclusive sets of dimensions. This assumes a perfect decomposition of the variables of the problem, which is not always possible. However, this assumption allows KABOOM to study the effects of problem decomposition as a measure of team specialization. The agents on a team are evenly distributed across the sub-teams. For example, a team of six agents might be organized as one flat team of six agents, two sub-teams of three, three sub-teams of two, or six individuals all working on independent aspects of the problem. Each sub-team is specialized in that it controls a subset of all the dimensions in the problem. Table 3.1 illustrates how a 6-agent team would divide a 6-dimensional problem amongst sub-teams for different amounts of team specialization.

To demonstrate the effect of specialization on performance, Figure 3.4 shows the optimal amount of specialization for 32-agent teams of organic composition. The bottom horizontal axis shows the number of agents per team, while the top horizontal axis shows the number of sub- 


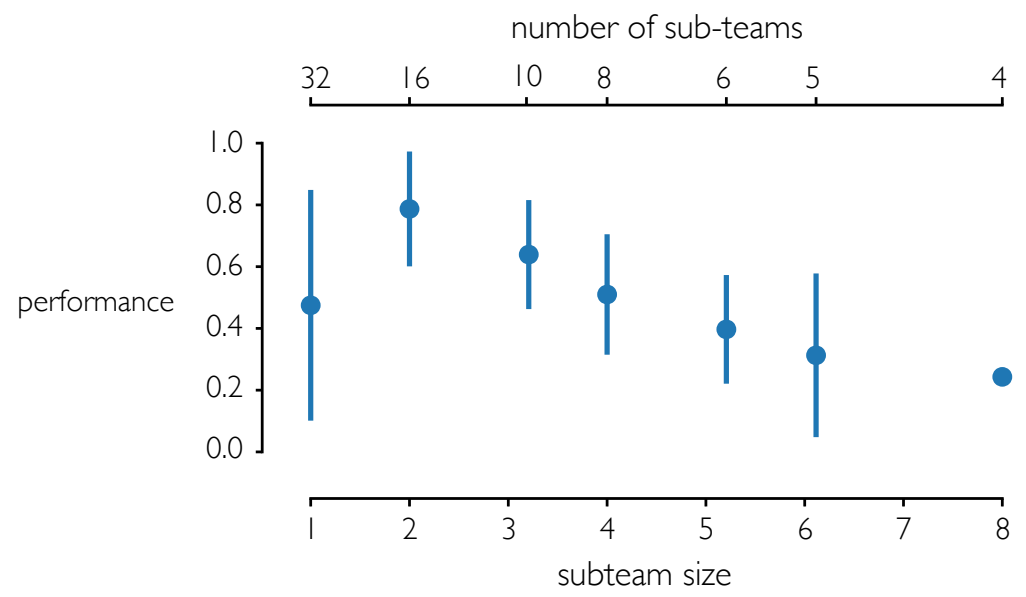

Figure 3.4. Performance of teams of 32 agents with organic composition for different levels of team specialization (error bars indicate \pm 1 standard deviation)

teams. (Team size and number of agents per team determine the number of sub-teams.) The optimal specialization for 32-agent teams or organic composition is sixteen sub-teams (pairs) of two agents.

The next investigation explores whether cognitive style affects the optimal amount of team specialization. We use the null hypothesis that the cognitive styles of agents on the team will not affect the optimal amount of specialization. Figure 3.5 shows the same study as above, but with homogeneous teams of more innovative, more adaptive, and mid-range styles rather than organically composed teams. The optimal specialization was significantly different for the three types of teams (Figure 3.5). The innovative teams perform best in larger sub-teams of four or eight agents, while the mid-range teams perform best with complete specialization (32 teams of one agent each), and the adaptive teams showed less dependence on specialization but perform best with three agents.

These results suggest that it may be important to consider the styles of team members when deciding how and how much to divide a team into specialized sub-teams. Specifically, the model predicts that a homogeneous team of more innovative individuals will perform poorly with small, highly specialized sub-teams. This result is consistent for problems with different values of $\alpha$ and $\beta$ (the objective function parameters). Complete specialization into 1-person sub-teams requires a high level of trust in each agent's performance, because every agent will contribute to the team's aggregate solution. However, innovative agents tend to perform less predictably (i.e., sometimes poorly and sometimes well), making it easier for smaller innovative sub-teams to go (and remain) off track. Having larger teams of four to eight innovators allows the full team to take the best solution of one agent on each sub-team and discard lower quality solutions.

Mid-range teams, on the other hand, perform best with complete specialization into oneperson sub-teams. These agents do not explore radical solutions in potentially damaging ways, but they do explore the space sufficiently to find good solutions. This trend reflects the results 


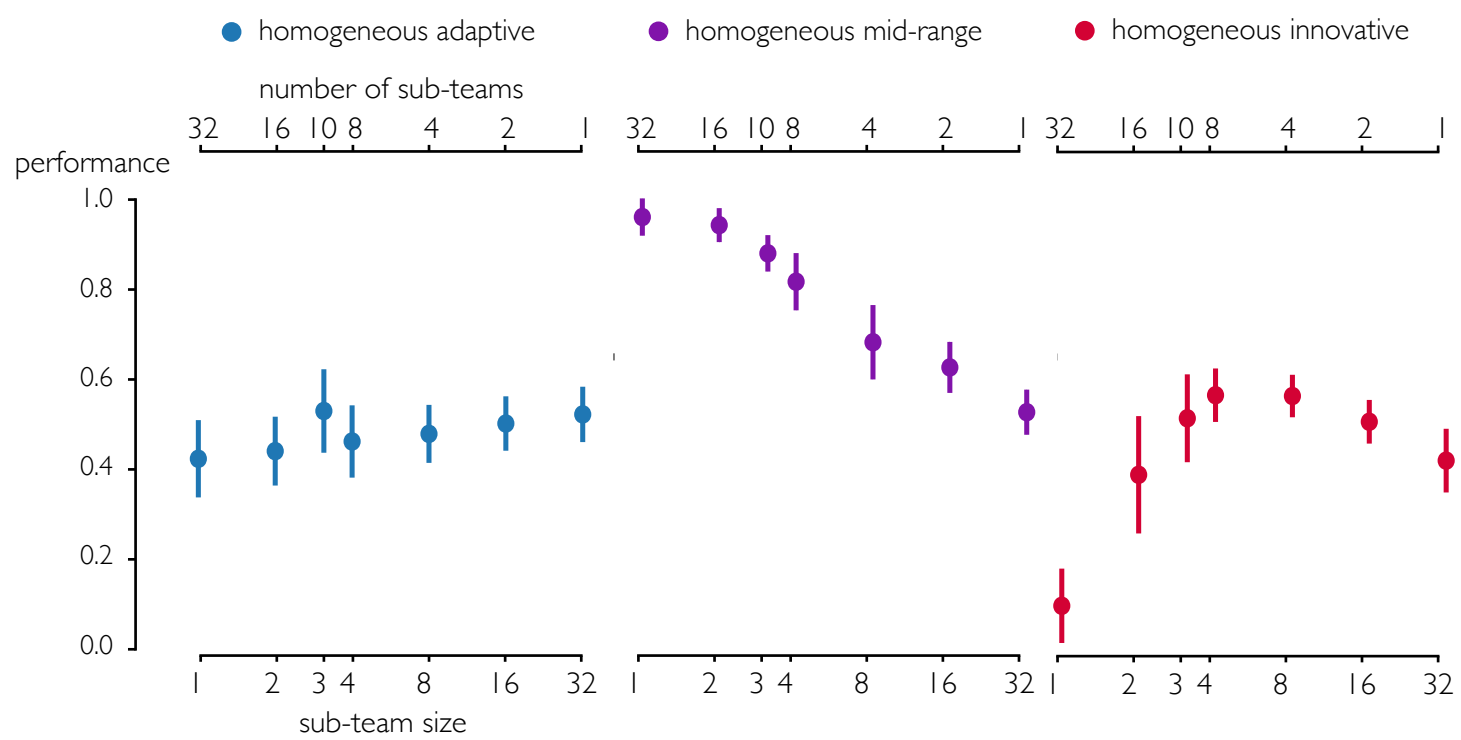

Figure 3.5. Plotting performance versus specialization for three homogeneous teams of different KAI styles shows that teams of innovators suffer when specializing into one or two person sub-teams while mid-range styles excel through specialization (error bars indicate \pm 1 standard deviation).

of Blackburn et al. [62], a study of software development teams which concluded that increasing team size decreased productivity. Finally, homogeneous adaptive teams do not explore radical solutions that could result in poor performance, but they also do not explore the solution space sufficiently to find high-quality solutions. The effect of team specialization on their performance is small, which is likely a result (once again) of the high degree of consistency found within adaptive teams due to their preference for consensual decision-making. In future work, human subjects studies will be needed to test the hypotheses suggested by all of these results - i.e., for more adaptive, more innovative, and mid-range teams of various amounts of specialization.

\subsubsection{Composition: Testing the Advantage of Style Diversity}

The methods section demonstrated how an individual agent's exploration of the design space in KABOOM depends on its cognitive style. In reality, the members of a design team are likely to have a range of cognitive styles, rather than all having the same style. However, it is unclear under what circumstances a wider diversity of styles on a team would aid or hinder the team's performance. This section examines how a team's diversity of cognitive styles affects performance on a set of problems.

\subsubsection{Performance of Homogeneous Style Teams on Diverse Problems}

This section analyzes the performance of homogeneous teams of different shared KAI scores on a matrix of 25 problems. These problems were created by modifying the objective function parameters - i.e., by permuting five logarithmically spaced values for $\alpha$ and five logarithmically 
spaced values for $\beta$.

The first parameter $(\alpha)$ affects the amplitude of the sinusoid (Figure 2.10). When the amplitude is large relative to the quadratic function, the quadratic becomes negligible, so that optimizing any local minimum yields similar performance to finding the global minimum. On the other hand, when the amplitude is small, the quadratic function becomes important in the cost function, so that finding the global local minimum is much better than finding a distant local minimum. Because large amplitude problems reward local exploration, while small amplitude problems reward global exploration, we hypothesize that more adaptive agents (lower KAI scores) will outperform other styles on large-amplitude problems (large $\alpha$ ), while more innovative agents (higher KAI scores) will have an advantage in small-amplitude (small $\alpha$ ) problems. The second parameter $(\beta)$ affects the size of the search space. Large search spaces require broader search strategies and more stochastic methods, corresponding to higher temperature in a simulatedannealing paradigm [54]. Therefore, we hypothesize that higher (more innovative) KAI scores will be advantageous for large search spaces (large $\beta$ ), while lower (more adaptive) KAI scores will have an advantage in small search spaces $($ small $\beta$ ), with intermediate KAI scores having an advantage in between.

Homogeneous teams of more adaptive, more innovative, and intermediate styles, respectively, were tested on the 25 problems. Each combination of $\alpha, \beta$, and shared KAI score was repeated only 8 times due to the large number of combinations. Figure 3.6 shows the optimal style of a homogeneous team on the KAI spectrum for each problem (from red as the most innovative homogeneous team to blue as the most adaptive homogeneous team). The optimal style of the problems ranges from highly innovative in the upper-left corner (large search space, small sinusoidal amplitude), to mid-range, to highly adaptive in the lower-right corner (small search space, large sinusoidal amplitude).

Both our hypotheses about the alignment of shared team cognitive style and problem characteristics proved to be correct: larger search spaces favor innovators, and larger sinusoidal amplitudes favor adaptors. The combined effect of the two problem variables is that extreme innovators perform best for problems with small $\alpha$ and large $\beta$ (lower right corner of the figure), extreme adaptors perform best for problems with large $\alpha$ and small $\beta$ (upper left), and intermediate styles perform best on other combinations of $\alpha$ and $\beta$. It is worth noting that there are some problems where having a mid-range style is advantageous over a more adaptive or more innovative style, which validates the importance of remembering that cognitive style is continuous, rather than a dichotomy of two "types" [5].

Figure 3.7 shows the results of the same study in more detail. For each problem in the 25-problem grid, performance is plotted versus the shared KAI score of agents on homogeneous teams of different styles. The red curve shows a quadratic regression fitted to the performance versus the KAI score for that problem. The value of KAI at the maximum of the quadratic function corresponds to the best KAI score reported for each problem in Figure 3.6.

Like these simulated problems, real life problems can favor one cognitive style over another. For example, problems that require adherence to a given structure, meticulous attention 


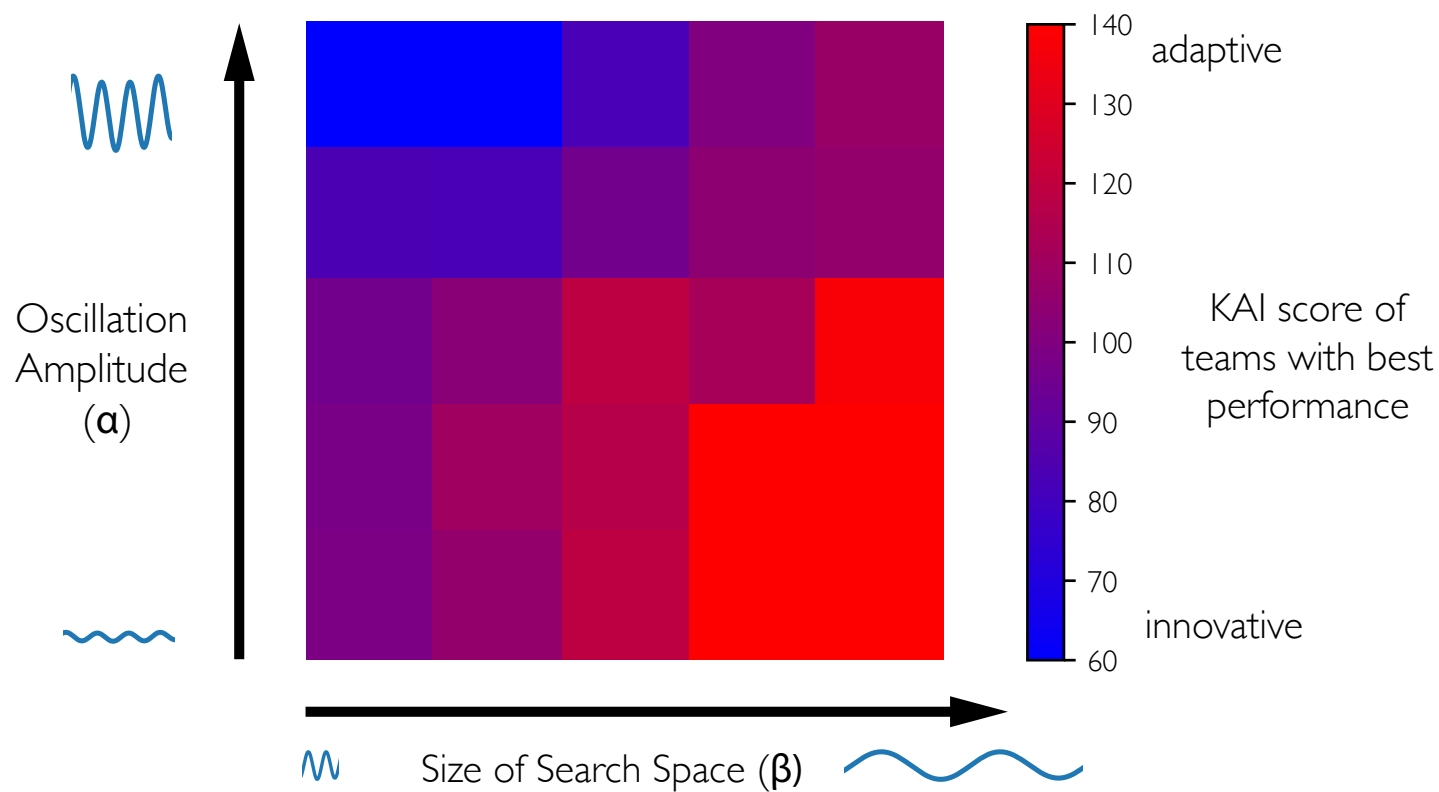

Figure 3.6. Homogeneous teams of different KAI styles were tested on each of 25 problems (represnted by squares), with varying $\alpha$ and $\beta$. Each square's color indicates the best-performing style for that problem from blue (more adaptive) to red (more innovative).

to detail, and conformance to specific rules or standards (e.g., repairing an antique grandfather clock, tuning a nuclear reactor) will tend to favor a more adaptive approach, although more innovative methods will still yield some kind of solution. In contrast, problems that require spanning multiple disciplines, taking a systems view, and challenging current practice (e.g., creating a disruptive product) will tend to favor a more innovative approach, although more adaptive methods will still lead to some kind of progress.

The $\alpha$ and $\beta$ parameters of the simulated problems are loosely related to characteristics of these real life problems. The oscillation amplitude $\alpha$ aligns with the importance of global exploration versus local exploitation. Real world design problems focused on new product development generally require broad exploration (as in designing a new children's toy), while redesigns and improvements of existing designs require thorough local exploration (as in improving the efficiency of an internal combustion engine). The effective solution space size $\beta$ corresponds to the number of iterations required to explore a space given a constant step size. Real world problems may have small solution spaces when there are few variables, when variables only take a limited number of discrete values, or when there are a small number of distinguishable solutions. Conversely, problems can have large solution spaces when they have many dimensions or continuous variables with many distinguishable values. For example, a traveling salesman problem [63] may have a small solution space if it only has a few nodes, but a very large solution space if it has many nodes. Future work will incorporate contextualized design problems in the model to better 


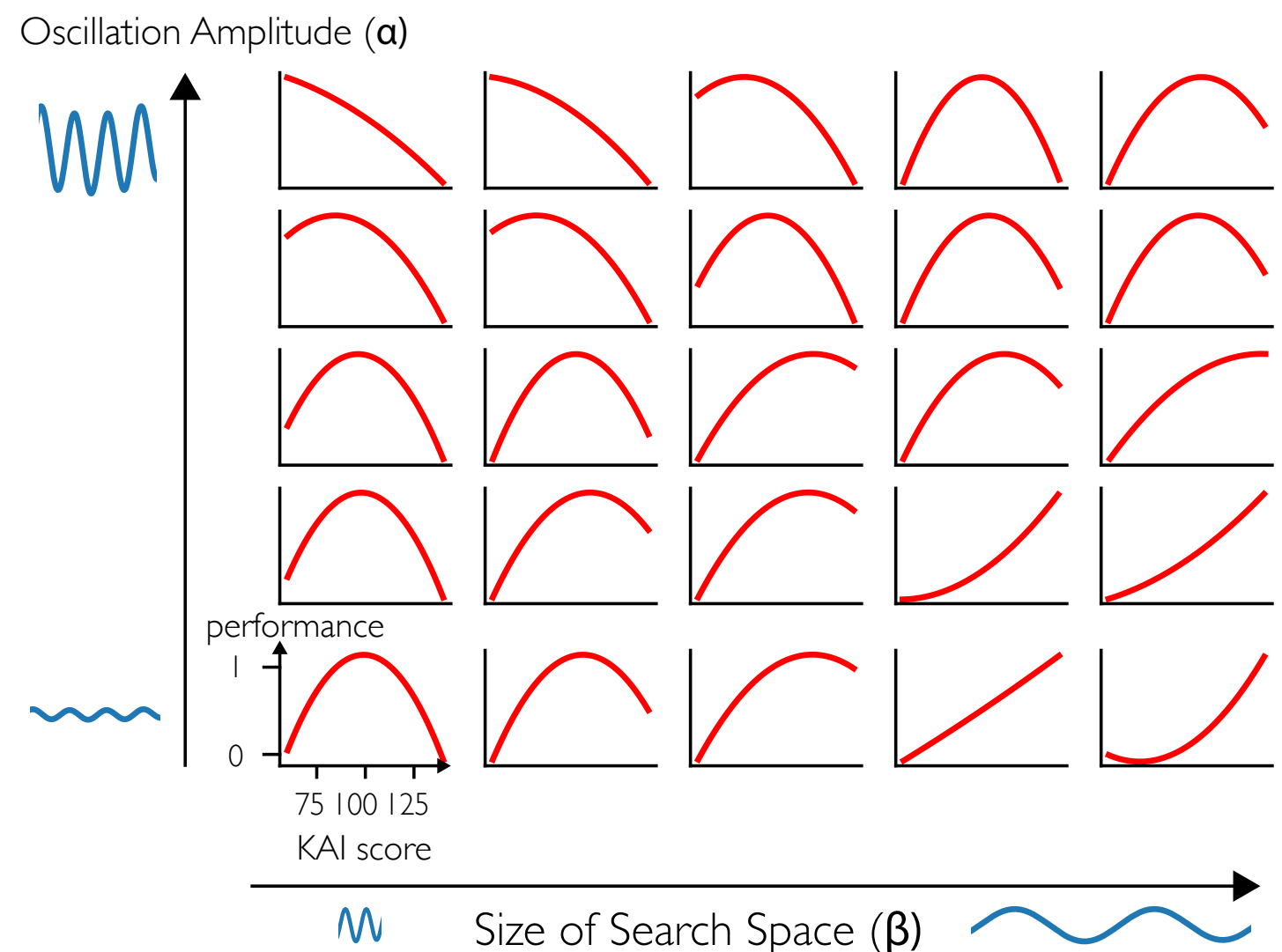

Figure 3.7. Each sub-plot shows a quadratic regression of performance versus KAI score for one of 25 problem variations (as in Figure 3.6). Lower KAI scores perform best in the upper left, and higher KAI scores perform best in the lower right.

understand the links between computational objective functions and real world problems.

\subsubsection{Performance of Heterogeneous Style Teams on Diverse Problems}

The literature relating various aspects of team diversity to performance shows mixed results. Some studies report that diversity of team members improves group performance in ideation and problem-solving tasks $[64,65,66,67]$. A number of recent studies have demonstrated that teams with ethnic, cultural, and gender diversity can outperform less diverse teams, at least in certain circumstances $[68,69,70,71,72]$. However, other literature concludes that diverse teams perform worse than homogeneous teams $[73,74,75,76]$. These studies focus on aspects of diversity such as race, gender, and culture. As there is no consensus on how these aspects of diversity impacts its performance, our investigation of cognitive style as an alternative measure of team diversity may yield insights into the factors that make diversity helpful or harmful to a team.

Here, we investigate how a team's cognitive style diversity can impact its performance. If a team must perform a range of problems, each of which favors a different problem-solving style, we hypothesize that heterogeneous linearly distributed teams will outperform homogeneous 


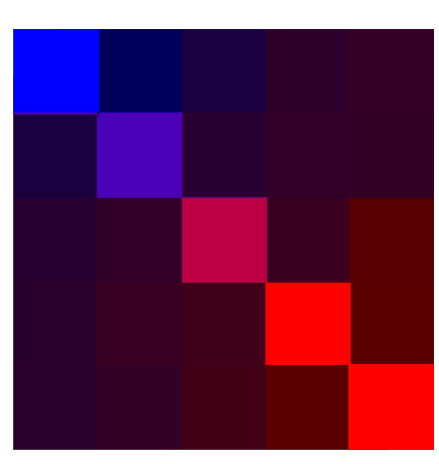

Problem Set 1

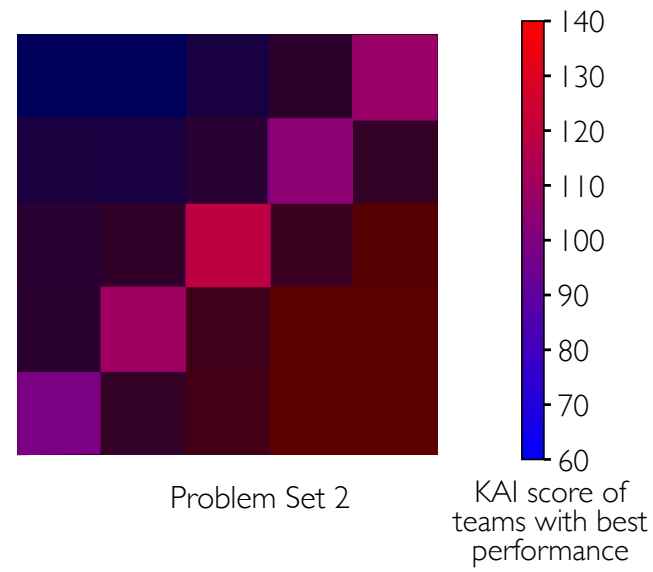

Figure 3.8. Of the 25 problems in figure 3.6, 5 were selected for each problem set. Color indicates the optimal KAI style for a problem. Problem Set 1 contains problems favoring a range of styles, while Problem Set 2 contains five problems favoring a mid-range style.

teams of mid-range style.

To test this, the teams were given a set of five problems selected from the 25-problem matrix. Two different problem sets are shown in Figure 3.8. The first problem set selects problems on the $\alpha \propto-\beta$ diagonal, so that the five problems favor a range of styles from more adaptive to more innovative. The second problem set, on the $\alpha \propto \beta$ diagonal, contains five problems that favor a mid-range style.

Figure 3.9 shows the average performance of teams with different KAI style ranges on the two problem sets. This experiment was repeated 32 (rather than 16) times to improve confidence in the results. In Problem Set 1, the heterogeneous linearly distributed teams with maximum cognitive gaps of more than 20 points outperformed the homogeneous mid-range teams, supporting our hypothesis that heterogeneous teams are advantageous when the team must tackle a wide variety of problems. A set of pairwise t-tests reveal that teams with maximum cognitive gaps of 40,60, 80, and 100 performed better $(p<0.001)$ than a homogeneous team (i.e., a team with a maximum cognitive gap of zero). Teams with a maximum cognitive gap of only 20 points did not perform significantly better than a homogeneous team $(p>0.5)$.

Interestingly, this transition at a 20-point gap corresponds to work by McCarthy [77], which reports that team stress due to cognitive style gaps begins to increase exponentially at 20 points. In McCarthy's work, there is a negative effect of increased stress on the team while this study shows a positive effect on performance. In either case, teams with a range of KAI styles within 20 points does not experience the same effects as those with larger differences in cognitive style.

For the second problem set, the style range of the team has only a small effect on performance except for extreme levels of diversity, which hurt performance. A set of pair-wise t-tests revealed that compared to a homogeneous team, a maximum cognitive gap of 20 or 40 points did not significantly impact performance $(p>0.5)$, while a maximum of cognitive gap of 60 

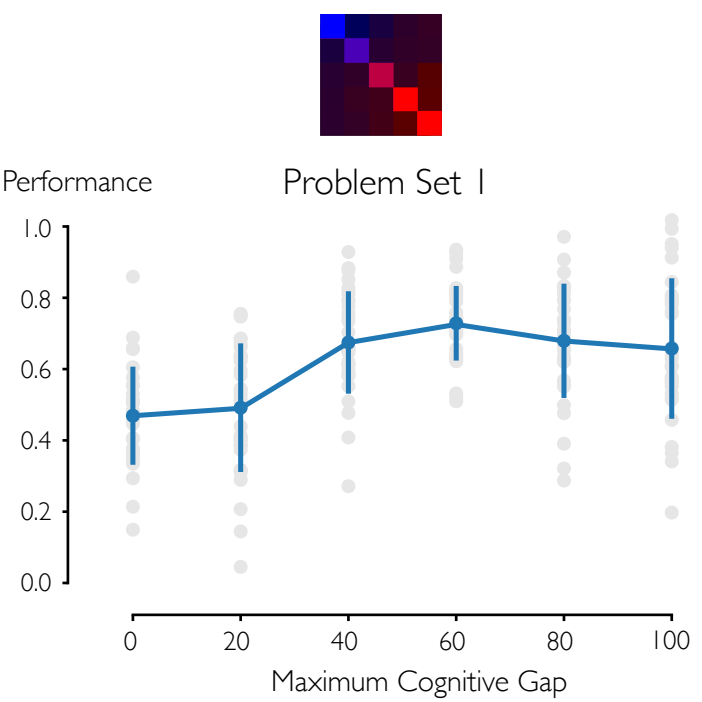

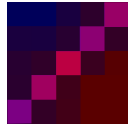

Performance Problem Set 2

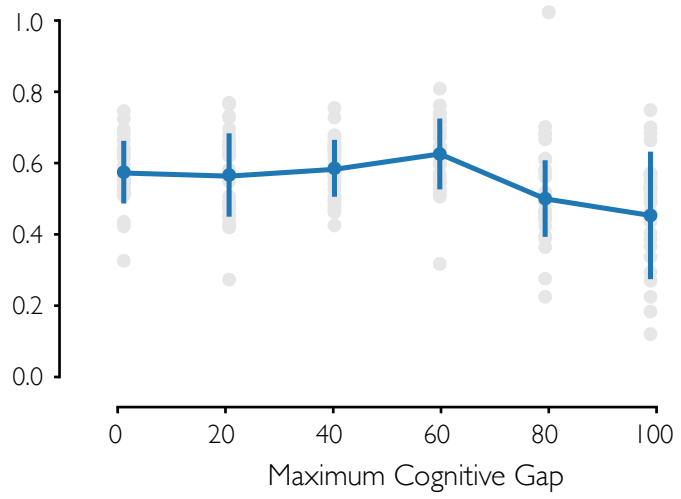

- mean performance of all teams

Figure 3.9. Performance of linearly-distributed heterogeneous teams is plotted for problem sets 1 and 2 (error bars indicate \pm 1 standard deviation). When problems in the problem set are suited for different styles (Problem Set 1), diversity of cognitive style benefits teams.

points led to a slight improvement in performance $(p<.05)$. A maximum cognitive gap of 80 or 100 points (close to the maximum observed range) caused a substantial decrease in performance $(p<.05)$. These results are not surprising, because the less diverse teams (smaller cognitive gaps) had more mid-range agents, and all five problems in the set favored generally mid-range styles. The two studies together suggest that teams composed of heterogeneous cognitive styles can sometimes have an advantage over homogeneous teams of uniform cognitive style, depending on the nature of the problems the team has to solve.

\subsection{Combined effects of Communication, Specialization, and Composition}

This section presents three studies on the interactions of communication, composition, and specialization on team performance. Comparing each pair of factors represents a pair-wise exploration of the three coordinate planes in a communication-composition-specialization coordinate frame. There are many ways to explore the interactions of any two factors such as communication and composition. This paper presents one exemplary study for each pair of factors. 


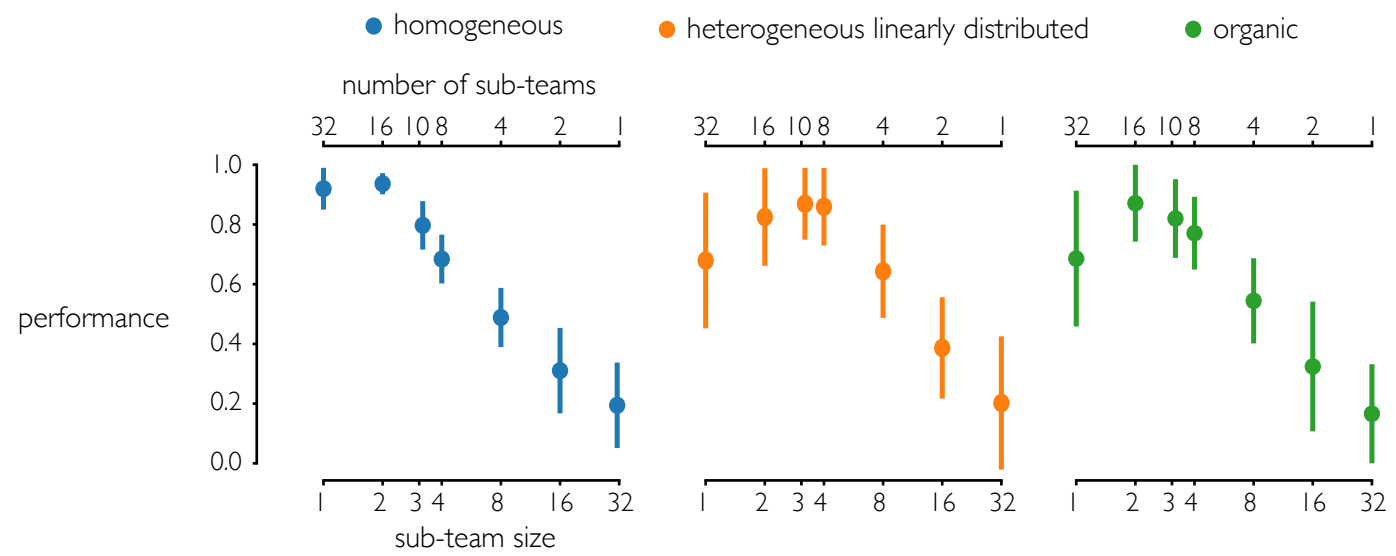

Figure 3.10. The optimal amount of specialization depends on a team's composition of cognitive styles. Error bars show \pm 1 standard deviation in performance.

\subsubsection{Composition and Specialization}

To compare the interaction of specialization and composition on team performance, this experiment tests the question: "For a given problem, do heterogeneous teams need a different amount of specialization than homogeneous teams?" To test this, the optimal-specialization study from earlier is repeated for teams with three different style compositions introduced in the methods section: (1) homogeneous mid-range teams (all agents have mid-range style); (2) heterogeneous linearly distributed; or (3) organic (randomly selected agents from a statistically representative population).

The results shown in Figure 3.10 show that team composition and specialization do have a non-trivial interaction. This experiment was repeated 32 (rather than 16) times to improve confidence in the results. The heterogeneous teams performed poorly for high amounts of specialization, as did organically composed teams. However, the homogeneous teams performed well with extreme specialization. These results agree with the results for homogeneous teams of different styles: trusting an innovative agent to work as an independent sub-team within a heterogeneous team is risky and results in poor performance. Even the presence of a few innovative agents on the heterogeneous linearly distributed and organic teams is enough to warrant larger sub-team sizes to help keep the full team on track. Thus, understanding the team members' cognitive styles is important for organizing the team into specialized sub-teams.

\subsubsection{Composition and Communication}

Next, an experiment tested the interaction of composition and communication in an attempt to answer the question "For a given problem, do teams of heterogeneous style require different amounts of pairwise communication than homogeneous teams?" One study of 53 technology development teams [78] reports that the social integration of teams is negatively correlated with communication rate; that is, teams that are less socially integrated require more communication 


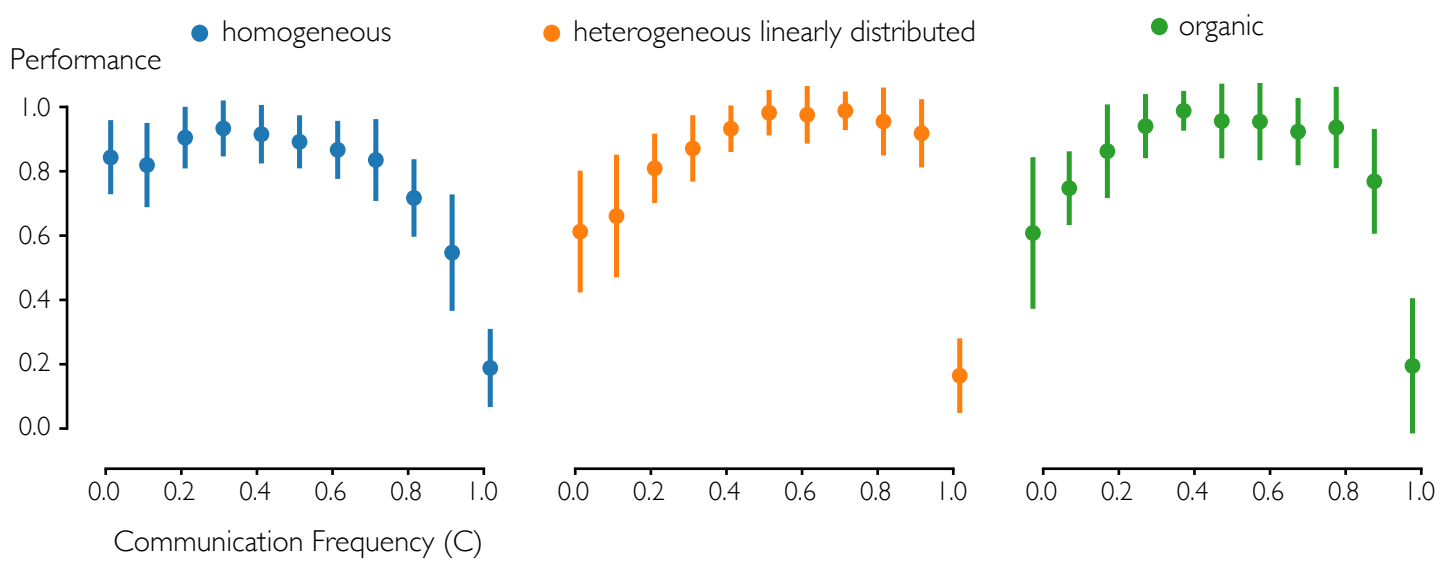

Figure 3.11. The optimal rate of pairwise communication depends on a team's cognitive style composition (12-agent team with 4 sub-teams). Error bars show \pm 1 standard deviation in performance.

while teams that are more socially integrated need only sparse and informal communication to succeed. Assuming that homogeneous teams are more socially integrated than heterogeneous teams, this suggests that the heterogeneous teams in KABOOM will have a higher optimal communication rate than homogeneous teams, and will not perform well with low communication rates.

This study compares the performance versus communication frequency trade-off for homogeneous teams and two types of heterogeneous teams (linearly distributed and organic, i.e. drawn randomly from the population). We hypothesize that diverse teams and organic teams will perform best with higher rates of communication than homogeneous mid-range teams (which perform best with $c=0.4$ ).

As shown in Figure 3.11, the results of our simulations support our hypothesis. The homogeneous team of mid-range style (blue) performs best at $0.3<c<0.4$, as before. The teams with a wide range of linearly distributed styles (orange) perform best with a communication frequency of $c=0.7$, and poorly with low communication rates. The teams with organic composition (green) perform best with $c=0.4$, but continue to perform well for higher values of $c$, like the heterogeneous teams and unlike the homogeneous teams.

These results agree with our hypotheses based on the results from Smith et al. [78]. Also, the performance of heterogeneous teams shows a mixture of the effects observed for homogeneous more adaptive, mid-range, and more innovative teams in Figure 3.2. Specifically, the heterogeneous teams reflect poor performance at low communication frequencies (as in homogeneous innovative teams) but also sub-optimal performance at high communication rates (as in homogeneous mid-range teams). This suggests that it may be possible to treat heterogeneous teams as mixtures of homogeneous teams to better predict optimized process characteristics. 


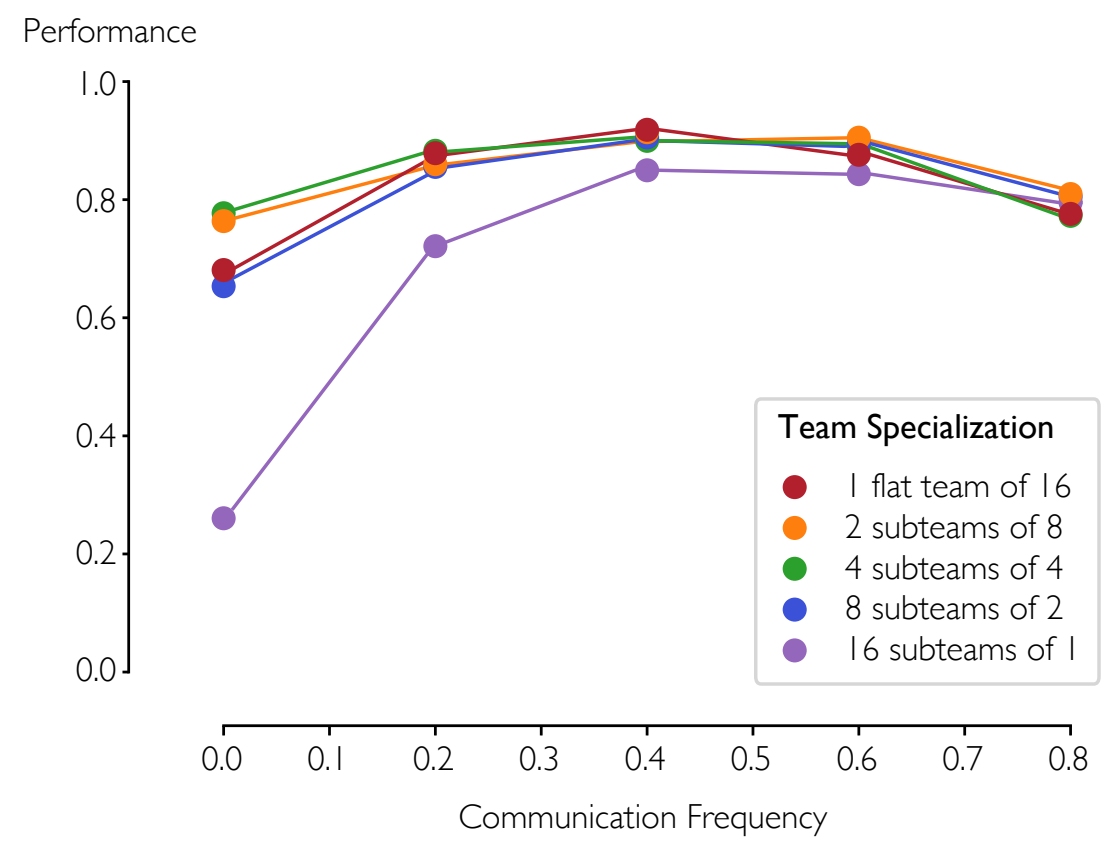

Figure 3.12. In these results, the best communication policy does not depend on team specialization for homogeneous teams of mid-range style. However, specialized teams perform poorly without communication.

\subsubsection{Specialization and Communication}

This section examines how a team's specialization into sub-teams interacts with communication by attempting to answer the question "Do specialized teams need different amounts of pairwise communication than flat teams?" The experiment tests different communication frequencies on one problem to determine the optimal rate of communication. Repeating this experiment for 16-agent teams with different amounts of specialization reveals the impact of specialization on optimal communication rate. Figure 3.12 shows that in general, changing the amount of specialization does not change the optimal amount of communication $c$, which is consistently 0.4. However, the poor performance of highly-specialized teams when $c$ is zero demonstrates that having no communication is especially detrimental for highly specialized teams. Teams of agents that completely specialize but do not communicate their solutions with each other were not able to converge effectively on coherent solutions.

The importance of communication in specialized teams is well recognized for software engineering projects. Brooke's Law [3] describes how large communication overheads in specialized software teams can make larger teams less productive. A more recent study showed specifically that the communication overhead can increase with the number of specialized teams [79]. The poor performance of the most-specialized teams without communication (Figure 3.12) reflect the importance of specialized teams spending time and effort on communication. 
CHAPTER 4

\section{Results: contextualized design problems}

\subsection{Research Questions}

Translating the results of computational experiments to a real-world context can be challenging, especially when the simulated problem and environment are abstract. Simulations should ideally reflect real-world problems and scenarios, so that their results can be compared directly to real teams. Some existing models of engineering teamwork incorporate contextualized, real-world problems [33, 1, eg], while others use more abstract objectives [51, 30, eg]. This chapter uses two contextualized problems (a simple beam-design problem and a multi-objective race car-design problem) in order to evaluate the performance of simulated teams in an agent-based model.

Four research questions guided the computational experiments in this chapter:

1. How do the cognitive styles of team members impact team performance on different contextualized problems?

2. In a specialized team, what are the optimal cognitive styles for each sub-team?

3. Can strategically assigning agents to sub-problems based on their cognitive style improve the team's performance?

4. How does changing the decomposition of a problem affect the team performance?

Here, we discuss the results of computational experiments that address these research questions by testing the performance of teams with differing cognitive style compositions. 

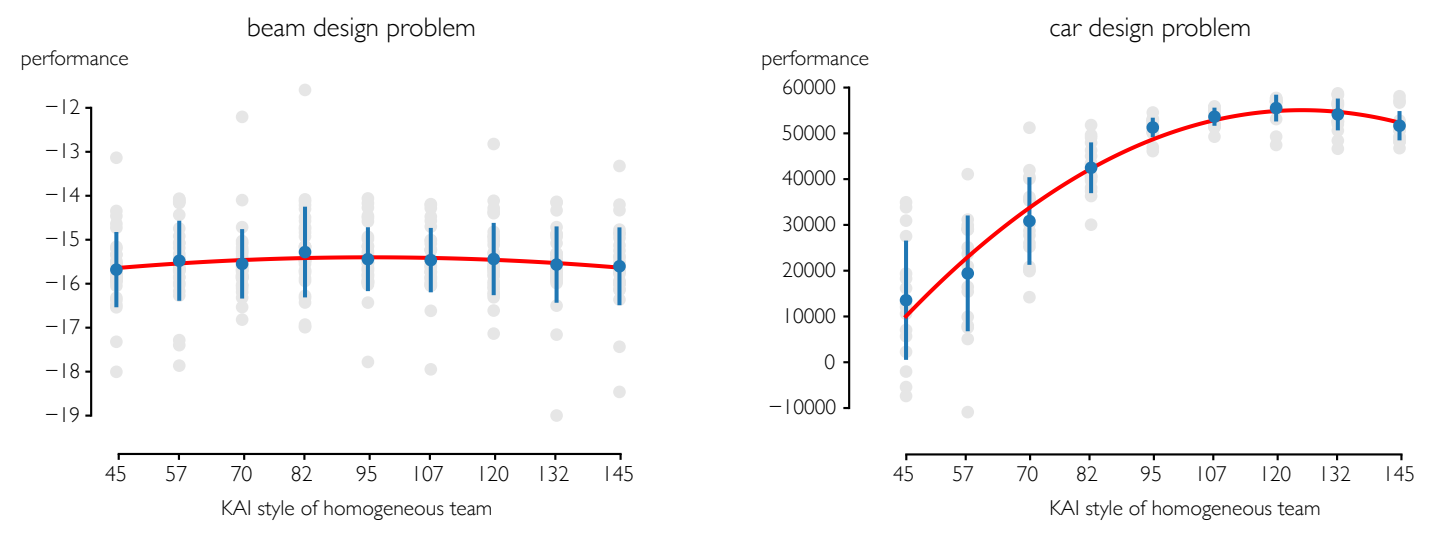

Figure 4.1. Style did not affect performance in the beam design problem. For the car design problem, teams composed of more innovative agents performed better.

Cognitive style is independent of cognitive level, and no cognitive style is better than another in general. Still, in some specific problems, individuals of certain cognitive styles may perform better than others. For instance, innovators may perform better than adaptors on design problems focused on new product development which require broad exploration (e.g. designing a new children's toy) while adaptors may perform better than innovators on improvements of existing designs, which require thorough local exploration (e.g. improving the efficiency of an internal combustion engine).

\subsection{Results}

\subsubsection{Cognitive Style on Two Problems}

The first computational experiment creates homogeneous teams (i.e., all agents on a team have the same cognitive style) and tests them on both the beam design and car design problems. For the beam design problem, this involved an 8-agent team in a 100-step simulation, specialized into 2 sub-teams of 4 agents each (each sub-team controls two of the four design dimensions). Figure 4.1 (left) shows that changing the shared cognitive (KAI) style of the homogeneous team did not impact performance on the beam design problem. The car design problem is significantly more complicated, with a 33-agent team decomposing the 56-variable problem into 11 sub-problems (11 sub-teams of 3 agents each), also with a 100-step simulation. For this problem, the shared cognitive style of the team had drastic effects on performance, with the more innovative teams outperforming the more adaptive and mid-range teams (Figure 4.1, right).

Though there are no human studies on these specific problems to compare our results to, the results demonstrate that some cognitive styles may be more or less effective for specific types of problems. Because the advantage of one style over another is problem dependent, a deeper understanding of cognitive style and its relationship to design problems is critical for appropriate managerial interventions within design teams. 


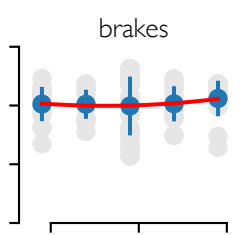

front wing

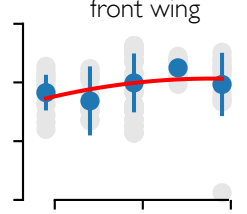

rear tire

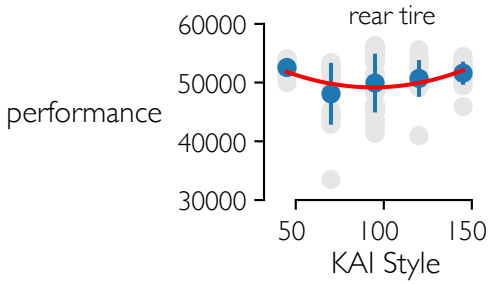

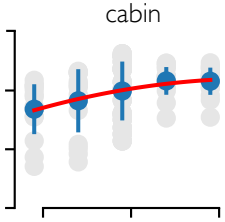
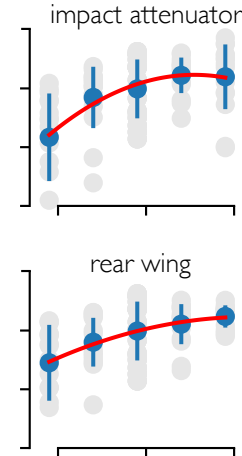
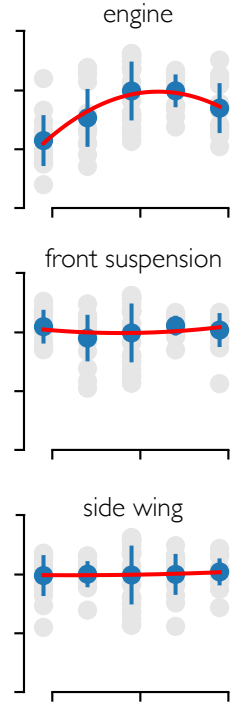
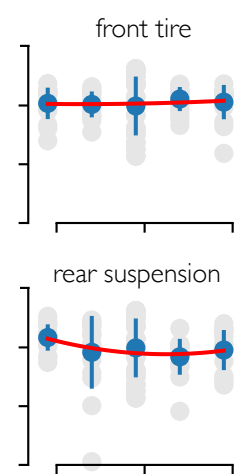

- quadratic fit

- mean performance

I \pm I standard deviation team performance

Figure 4.2. Varying the style of one sub-team while holding the rest of the team constant (homogeneous mid-range) revealed that depending on the sub-problem, one KAI style can perform better than others.

\subsubsection{Cognitive Style on Specialized Team of the Race Car Problem}

In order to better understand how a team's cognitive style composition impacts performance in the race car design problem, our second experiment tests the sensitivity of each specialized sub-team to changes in cognitive style. All remaining experiments utilize the more complicated race car design problem. For each of the eleven sub-teams, the sub-team's style is varied while holding the style of the rest of the remaining sub-teams constant (all agents outside the sub-team in question have a mid-range style of 95). The results in Figure 4.2 demonstrate that each subteam responds differently to changes in its cognitive style composition. For example, populating the impact attenuator team with more innovative agents improves overall team performance, while populating the rear suspension sub-team with more adaptive agents improves performance, and mid-range agents are the best style for the engine team. KABOOM does not model any aspect of domain expertise, and these results do not suggest that cognitive style is related to the level of expertise or ability for designing a certain aspect of the car. Rather, the results suggest a meaningful difference in the characteristics of the solution space for different parts of the car problem. For instance, the solution space explored by agents designing the rear suspension (where adaptive agents performed best) may be best suited for the incremental, detailed, "adaptive" search approach, while the solution spaces explored for the rear wing and impact attenuator (where innovative agents performed best) may be better suited for a more stochastic, "innovative" problem-solving approach. 


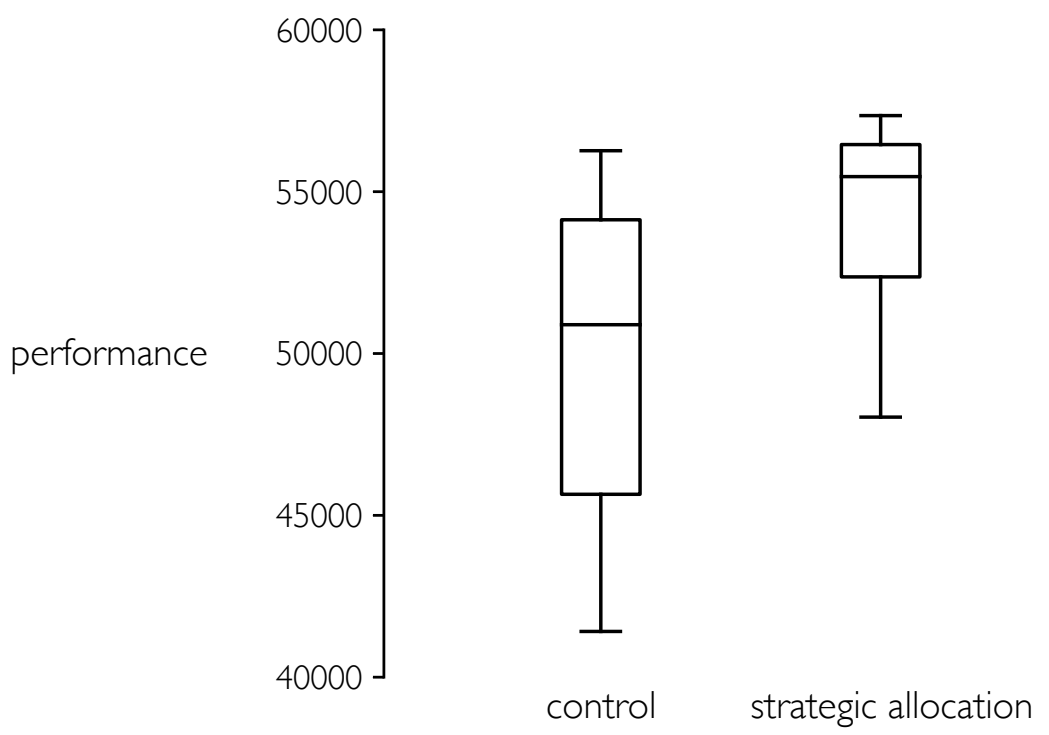

Figure 4.3. When a team is composed of a random selection of cognitive styles, strategically assigning agents to sub-problems based on cognitive style improves performance significantly.

\subsubsection{Strategic Task Assignment Using Cognitive Style}

Given that the sub-teams of the race car design problem had a variety of optimal cognitive styles, we can strategically assign agents to the sub-problems that are best-suited for their respective styles. The following experiment uses teams with organic composition, which means they are composed of agents randomly drawn from a virtual population. The virtual population reflects a realistic distribution of cognitive styles based on a data set of 597 individuals' KAI scores and sub-scores gathered in previous research [20]. In the control group teams, agents from the organic team are randomly assigned to sub-teams without regard for their cognitive style. In the experimental group of strategic allocation teams, agents are assigned to sub-teams based on their cognitive style. First, the agents are listed in order of ascending cognitive style, and the subteams are listed in order of ascending cognitive-style preference (accounting for the strength of the style effect, as well as the best style). This ordering is: Rear Suspension, Rear Tires, Brakes, Side Wing, Front Tire, Engine, Front Suspension, Front Wing, Cabin, Impact Attenuator, and Rear Wing. Then, the agents are assigned to the sub-teams according to the ordered lists (e.g., first three agents to first sub-team, etc). Figure 4.3 shows that the strategic teams outperformed the control group teams significantly (effect size $=1.12, \mathrm{p}<.05$ ).

Teams in both the control and strategic sets are composed of randomly chosen agents with respect to cognitive style. This demonstrates that strategically placing the right members of the team on the right sub-teams could significantly improve performance. This result is supported by a human study of team success and KAI cognitive diversity by Hammerschmidt [36], which found that teams had higher levels of success when tasks were coordinated with team members' KAI style. In real design teams, dimensions of cognitive level (e.g., disciplinary knowledge, experience, 


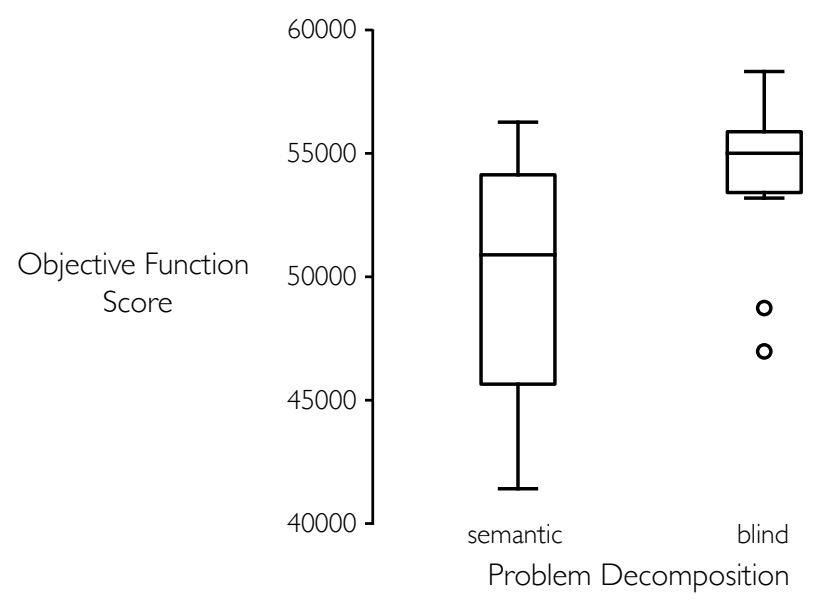

Figure 4.4. Assigning variables of the car problem to sub-teams randomly resulted in better performance than using the semantic problem decomposition.

intelligence) are important factors in assigning members of a team to different parts of a problem. However, all else being equal, accounting for cognitive style when selecting people for different parts of a problem could improve a team's performance. This result also demonstrates that having team members with diverse cognitive styles can be an advantage, rather than a disadvantage, for a team.

\subsubsection{Modifying Problem Decomposition}

As demonstrated in Figure 2.11, the variables of the race car design problem are decomposed according to the real-world application (i.e., wheel variables "belong together" and cabin variables "belong together"). This leads to an uneven distribution of the variables across the eleven subteams. The fourth experiment compares this "semantic" problem decomposition to a "blind" problem decomposition, where the design variables are evenly and randomly distributed among eleven teams without grouping related variables (i.e., one team may design the rear wheel radius, cabin thickness, and angle of the front wing). The experiment held the number of agents (33), number of sub-teams (11), team composition (homogeneous mid-range KAI style of 95), and number of iterations (100) constant. The results in Figure 4.4 show that teams that used the blind problem decomposition significantly outperformed teams that used the semantic problem decomposition based on the car's systems (effect size $=1.10, \mathrm{p}<.05$ ). In a human team, semantic problem decomposition would be critical to success, because problem solving depends on domain expertise and contextual knowledge. In the current KABOOM model, agents problem solve with no contextual or domain knowledge, which leads to the unrealistic boost in performance caused by redistributing the design variables. This suggests that incorporating some aspects of cognitive level (e.g., domain knowledge) in KABOOM is an important direction for future work. However, it also highlights the importance of equally distributing the human resources of a team across sub-teams in order to improve performance. 
CHAPTER 5

\section{Conclusions}

\subsection{Conclusion}

This thesis introduced KABOOM, the first team simulation modeling framework to reflect the diverse range of human cognitive styles. In KABOOM, the behaviors of heterogeneous agents simulate the range of cognitive styles described by the Kirton Adaption-Innovation spectrum. The primary objectives of this research were to create an agent-based model incorporating cognitive style, analyze the effects of style on problem-solving performance, explore the interactions of style with team composition, specialization, and communication, and investigate how teams can use cognitive style diversity to their advantage. The development of cognitive style in the simulated agents took inspiration from research in psychology, sociology, design science, and Kirton's Adaption-Innovation theory to reflect empirically observed human behaviors. The resulting model successfully simulated a range of distinct behaviors driven by cognitive style. In KABOOM simulations, the behavior of more adaptive, mid-range, and more innovative agents generally reflected the intended behavior and resulted in patterns in performance that were sometimes non-intuitive.

KABOOM was designed to allow the researcher to vary three primary factors of team organization: team-member style composition, specialization into sub-teams, and communication patterns.

In Chapter 3, KABOOM allowed us to explore the effects of team specialization, composition, and communication as they related to cognitive style and performance. A study of communication frequency revealed that teams of innovators would benefit from high communication 
frequencies that would be detrimental to mid-range or adaptive teams. This result demonstrates a trade-off of exploration and exploitation: innovators easily explore many solutions but must communicate frequently to converge and refine one solution; the mid-range agents did not have difficulty converging and needed to spend most of their time exploring; adaptors converge easily thanks to less solution disparity, but may not thoroughly explore the solution space.

With regard to team structure, innovative teams performed poorly with high levels of specialization, while mid-range teams performed better with more specialization, and adaptive teams showed consistent performance across different team structures. The unpredictable quality of a single innovator's work makes it risky to entrust a sub-problem to a one or two-person subteam and safer to choose the best solution to the sub-problem from a small group. Finally, testing teams on a set of problems revealed that diversity of cognitive style can benefit teams, depending on the problems they address. Diverse teams had an advantage when addressing a set of problems where each problem favored a different cognitive style. The advantage of diversity disappeared for a problem set where each problem favored a mid-range style.

Experiments that investigated the interaction of team composition and specialization suggested that understanding the diversity of cognitive styles on a team could inform the optimal level of problem decomposition. Specifically, teams with homogeneous style were able to specialize to a higher degree than heterogeneous teams. The optimal communication frequency for teams of different composition reflected the desired communication frequencies of their team members. Also, we found that the importance of communication increased dramatically when teams specialized completely into one-agent sub-teams. These experiments only scratch the surface of KABOOM's capability to explore the interactions of composition, specialization, and communication.

In Chapter 4, contextualized problems helped to make the results of the model more grounded and intuitive. Testing a range of cognitive styles on two different contextualized problems demonstrated that different cognitive styles may be more or less effective for different problems. Some of the decomposed sub-problems of the race car design problem were best suited to adaptive style, while others were best suited to innovative or mid-range style. Using that knowledge of the sub-problem, virtual teams were able to significantly improve performance by assigning agents to sub-problems that suited their cognitive style. This demonstrated that having a diverse set of cognitive styles on a team can be advantageous when the team tackles a complex problem. Finally, varying the decomposition of the problem revealed that agents performed better on a random problem decomposition rather than the semantic problem decomposition. This highlighted the lack of contextual knowledge or expertise of agents in the current model.

While this work has focused on developing a flexible simulation framework, future work will validate, and further develop, the model through a targeted set of human studies. The most powerful models and insights are likely to result from a hybrid research approach that merges the breadth and flexibility of computational simulation with the rich holistic results of human studies. The human studies will not only test the validity of insights provided by the model, but will also serve as a feedback loop for tuning and improving the behavior of agents in the 
model. Likewise, insights from the model will guide the development of human studies designed to examine specific hypotheses about behavior, teamwork, and cognitive style, which could lead to novel insights on social and cognitive behaviors. In time, the mutual feedback between in vivo and in silico scientific investigations will lead to a deeper understanding of the cognitive processes underlying design, and how those processes influence the behaviors of individuals and teams. By allowing computational agents to embody unique cognitive styles, this model opens the door to a future of design analysis built at the intersection of computers' precision and humans' individuality.

\subsection{Limitations}

The KABOOM model focuses on specific characteristics of cognitive style and team problem solving, and does not create a comprehensive representation of team problem solving. Because of this, there are several major limitations of the current model. With regard to cognitive style, the model attempts to map specific human problem-solving characteristics to parameters in the model (i.e. mapping the diversity of people's solutions to the step size and temperature in a simulated annealing framework). Cognitive style and behavior are extremely complex, and many important effects of style have not been modeled. For instance, KABOOM does not attempt to model the coping behaviors that people of different cognitive styles use when working together. Because modeling all aspects of human behavior would be unfeasible, we were forced to choose which aspects of behavior were most important to team performance outcomes, and envision how they would map onto quantitative parameters in the model.

The model's reflection of communication, collaboration, and specialization are also limited. Previous work has focused extensively on modeling communication and collaboration within teams $[31,30,11,40,41,34]$, resulting in models with much more complex mechanisms for communication than those in KABOOM. In real teams people communicate ideas about process, strategy, sentiment, and emotion, while agents in KABOOM only communicate their solutions. While more complex models of communication could be incorporated into KABOOM, the current mechanisms implemented (pair-wise solution sharing and team convergence with meetings) were

sufficient to reproduce the communication trade-offs observed in literature. Likewise, previous work has developed more complex constructs for team specialization that incorporate communication patterns, domain knowledge, and heterogeneous cognitive abilities [31, 32, 44]. In KABOOM, specialization is treated simply as a problem-decomposition, which may be an over-simplification of how organizational structure shapes team performance.

Further, this work only tests KABOOM with one abstract design problem and two contextualized problems. KABOOM is capable of simulating team performance on any problem that is expressed as an objective function of continuous variables. In the future, it would be useful to implement problems that can be mirrored in human-subjects studies so that simulation and study results can be compared directly.

The lack of any human subjects research to test the validity of results from the model 
is another important limitation of the current work. Without support from human-subjects validation studies, the results presented in this paper cannot be assumed to hold true in reallife scenarios. Future work will focus on validating model results with human-subjects studies. All of these limitations are a reminder that this model is an early experiment in the direction of simulating human cognitive style with agent-based modeling. The results from this new model are as much demonstration of the method's capabilities for studying teamwork as they are predictions of real-world behavior. Its limitations lay the groundwork for the future work described below.

\subsection{Future Work}

$\mathrm{KABOOM}$ is a flexible framework for studying teams with a focus on cognitive style that can and should be adjusted, expanded, and validated. This section suggests directions for future work for time frames of six months, three years, and ten years.

\subsubsection{Six Month Time Frame}

The direction of work on KABOOM in the next six months should include (1) implementing a new contextualized problem and (2) preliminary model validation through human-subjects studies. In order to compare results of the simulation with human-subjects studies, the design problem should be identical for both. Because of the time and personnel constraints of a human-subjects study, the race-car design problem is not appropriate. The design problem should be suited for a team of roughly 2-6 people with roughly 1-3 hours to complete. To work in KABOOM, the problem should ideally have a natural mapping onto a continuous variable space of several (roughly 10-100) variables. Ideally, this problem would include a natural problem decomposition where different pieces of the problem are best suited for different cognitive styles. That would allow for an experiment similar to Figure 4.3 where tasks are assigned to team-members either randomly or based on cognitive style. Implementing a new contextualized problem and running preliminary human subjects studies in the near future is critical for validating (and adjusting) $\mathrm{KABOOM}$ as a model of human behavior.

\subsubsection{Three Year Time Frame}

Future work in the three year time frame should focus on refining and enhancing the behavior of agents in KABOOM or subsequent models to better reflect human problem-solving behavior. Conducting human-subjects studies and comparing the results to the simulation will reveal the exact behavioral adjustments that are most critical to address in KABOOM. If it becomes clear that the simulated annealing framework and optimization-landscape approach used in KABOOM do not reflect human problem-solving behavior, KABOOM should be discarded in favor of a new model. If the model is reasonably close to reflecting human problem solving, then it may be worth refining the agents' specific behavioral rules and cognitive processes. For instance, if domain knowledge and expertise is a critical missing factor, agents could be given some pre-established 
knowledge or capacity to learn about a problem. Social behaviors and communication may be the most critical factors to improve, because the current model has no network structure (in KABOOM any agent communicates with any other agent without preference) and the only form of communication is the transfer of solutions. Many features would be relatively simple to add to the model, but to preserve simplicity features should only be added if they address a critical deviation of the model from real behavior.

The implementation of cognitive style could also be modified to better reflect observed behaviors of people with various KAI scores and sub-scores. The behavioral style rules in KABOOM are largely based on intuition and thought-experiments conducted by myself, Dr. McComb, and Dr. Jablokow. While the current implementation of cognitive style seems to make sense, we have not tested the problem solving behaviors of our stylized agents against actual people with known KAI scores in an experimental setting. It may also be necessary to revisit some of the early assumptions of the model, for instance: agents pick a search direction randomly; agents can move to a solution shared with them instantly; agents perceive their team's position as a centroid of their teammates positions; group meetings always result in convergence to a composite solution, and only require one iteration in the simulation; and many others.

Finally, re-implementing the model in $\mathrm{C}$ may become necessary for the performance boost. I have found the flexibility and prototyping-friendliness of Python to be useful. Though I spent some time streamlining the model to lower computation time, it is by no means optimal and could be improved while still using Python if simulation speed becomes important.

\subsubsection{Ten Year Time Frame}

In the ten year time frame, we can take a wider view of the topic of modeling cognitive style. Given the progress in artificial intelligence over the past ten years, we can expect to see large advances in not only computational problem solving and language processing, but also computational creativity and pseudo-human constructs of emotion and sentiment by 2030. As artificial intelligence takes over many traditional jobs, the most significant questions to address in this vein of research may be about finding the value of humans in a computer-centric world. As the children and grand-children of models like KABOOM become more and more human-like in some aspects of their behavior, they will also reveal what parts of human nature don't come naturally to a machine.

When Alan Turing invented a machine that could crunch numbers faster than hundreds of well-trained human "computers", it forced humanity to step back and reflect on our own value. We were no longer the most powerfully analytic and deductive problem solvers on Earth - that title had been snagged by a heartless mechanical contraption. But we shifted our perspective, and found value in our ability to work together and think creatively. Now, computers may be poised to overtake us on those fronts as well. But I think that the individuality of humans, seen in our cognitive styles and our personalities, will always hold value that artificial intelligence cannot replace. Perhaps this research is, in the big picture, about gaining a better understanding 
of the strengths and weaknesses of artificial intelligence and human beings. It's certainly about each learning from the other. In a ten-year time frame, we will be challenged to define a new relationship with technology, hopefully a collaborative one. If there is one takeaway from this thesis, it's that individuals with different strengths and weaknesses can collaborate to create synergy, and be stronger together than separate. Perhaps a partnership between artificial intelligence and

human beings can leverage the unique strengths of each to create something greater than either can be alone.

\subsection{Acknowledgements}

This research was supported with funding from the School of Engineering Design, Technology, and Professional Programs at The Pennsylvania State University. 
APPENDIX A

\section{Parameters Table}

Table A lists the parameters used for each figure in Chapter 3. The value var indicates that the parameter was an independent variable in the figure. Style Composition is indicated by $h$ (homogeneous) or o (organic). Except when noted explicitly in the figure, homogeneous teams had a KAI score of 95 (the observed mean). 
Table A.1. Model parameters for all results using the abstract problem.

\begin{tabular}{lccccccccccc}
\hline Parameter & \multicolumn{1}{c}{ Chapter 3 Figure number: } \\
& 3.1 & 3.2 & 3.3 & 3.4 & 3.5 & $3.6-7$ & 3.9 & 3.10 & 3.11 & 3.12 \\
\hline Team & & & & & & & & & & \\
\hline Team Size & 12 & 12 & 20 & 32 & 32 & 6 & 6 & 32 & 12 & 16 \\
Num Sub-Teams & 4 & 4 & 4 & var & 2 & 2 & 2 & var & 4 & var \\
Agents/sub-team & 3 & 3 & 5 & var & var & 3 & 3 & var & 3 & var \\
Style Composition & $\mathrm{h}$ & $\mathrm{h}$ & $\mathrm{o}$ & $\mathrm{o}$ & $\mathrm{h}$ & $\mathrm{h}$ & $\mathrm{h}$ & $\mathrm{var}$ & var & $\mathrm{h}$ \\
\hline Agents & & & & & & & & & & \\
\hline Avg. start temp & 1 & 1 & 1 & 1 & 1 & 1 & 1 & 1 & 1 & 1 \\
Stdev. temp & 0.8 & 0.8 & 0.8 & 0.8 & 0.8 & 0.8 & 0.8 & 0.8 & 0.8 & 0.8 \\
Avg. start speed & .07 & .07 & .07 & 0.01 & 0.01 & var & var & 0.01 & .07 & .07 \\
Stdev. Speed & .007 & .007 & .007 & .007 & .007 & var & var & .007 & .007 & .007 \\
\hline Communication & & & & & & & & & & \\
\hline Comm Freq. & var & var & 0.2 & 0.2 & 0.2 & 0.2 & 0.2 & 0.2 & var & var \\
Meeting Period & 50 & 50 & var & 50 & 50 & 50 & 50 & 50 & 50 & 50 \\
\hline Objective Func. & & & & & & & & & & \\
\hline Dimensions & 12 & 12 & 20 & 32 & 32 & 10 & 10 & 32 & 12 & 10 \\
Amplitude & 0.025 & 0.025 & 0.025 & 1 & 1 & var & var & 1 & 0.025 & 0.025 \\
Scaling & 1 & 1 & 1 & 1 & 1 & var & var & 1 & 1 & 1 \\
Iterations & 300 & 300 & 300 & 300 & 300 & 300 & 300 & 300 & 300 & 300 \\
Repetitions & 16 & 32 & 16 & 16 & 16 & 8 & 32 & 32 & 16 & 16 \\
\hline
\end{tabular}




\section{Bibliography}

[1] Zurita, N., M. Colby, I. Tumer, C. Hoyle, and K. Tumer (2017) "Design of Complex Engineered Systems Using Multi-Agent Coordination," Journal of Computing and Information Science in Engineering, 18(October), pp. 1-13.

[2] Johnson, J. (2005) "Better Together," in In Between Dreams, Brushfire Records.

[3] Brooks, F. P. (1975) "The mythical man-month : essays on software engineering," , p. 195.

[4] Seuss, D. (1959) Happy Birthday To You!, Random House.

[5] Kirton, M. J. (2003) Adaption-innovation: In the context of diversity and change.

[6] Picasso, P., "Quote by Pablo Picasso: The chief enemy of creativity is good sense.," .

[7] Hadjeres, G., F. Pachet, and F. Nielsen (2016) "DeepBach: a Steerable Model for Bach Chorales Generation," in Proceedings of the 34th International Conference on Machine Learning-Volume 70, 1612.01010.

[8] Zemeckis, R. (1994), "Forrest Gump," .

[9] Card, O. S. (1985) Ender's Game, Tor Books.

[10] McPhail, D. M. (1993) Santa's Book of Names, Little Brown Books for Young Readers.

[11] Patrashkova-Volzdoska, R. R., S. A. McComb, S. G. Green, and W. D. CompTON (2003) "Examining a curvilinear relationship between communication frequency and team performance in cross-functional project teams," IEEE Transactions on Engineering Management, 50(3), pp. 262-269.

[12] Theater, D. (2002) "The Test That Stumped Them All," in Six Degrees of Inner Turbulence, Elektra Records.

[13] Carnabuci, G. and B. Dioszegr (2015) "Social Networks, Cognitive Style, and Innovative Performance: a Contingency Perspective," Academy of Management Journal, 58(3), pp. 881-905. 
[14] Angelou, M. (2014) Rainbow in the Cloud, Random House.

[15] Tolkien, J. (1994) The Two Towers, Houghton Mifflin Company, New York.

[16] Floyd, P. (1973) "Time," in The Dark Side of the Moon, Harvest Records.

[17] Seuss, D. (1957) How the Grinch Stole Christmas!, Penguin, Random House.

[18] Harris, F. (2005), "In Conversation, "It's not over 'til its over"," .

[19] Riding, R. J. (1997) "On the Nature of Cognitive Style," Educational Psychology, 17, pp. $29-49$.

[20] Jablokow, K. (2008) "Developing Problem Solving Leadership: A Cognitive Approach." International Journal of Engineering Education, 24(5), pp. 936-954.

[21] Jablokow, K. W., W. Teerlink, S. Yilmaz, S. R. Daly, and E. M. Silk (2015) "The impact of teaming and cognitive style on student perceptions of design ideation outcomes," ASEE Annual Conference and Exposition, Conference Proceedings, 122nd ASEE(122nd ASEE Annual Conference and Exposition: Making Value for Society).

[22] Sonalkar, N., K. Jablokow, J. Edelman, A. Mabogunje, and L. Leifer (2017) "Design Whodunit: The Relationship Between Individual Characteristics and Interaction Behaviors in Design Concept Generation," in Volume 7: 29th International Conference on Design Theory and Methodology, ASME, p. V007T06A009.

[23] Kirton, M. (1976) "Adaptors and innovators: A description and measure," Journal of Applied Psychology, 61(5), pp. 622-629.

[24] Jablokow, K. W. and D. E. Bоотн (2006) "The impact and management of cognitive gap in high performance product development organizations," Journal of Engineering and Technology Management - JET-M, 23(4), pp. 313-336.

[25] Jablokow, K. W., J. F. Defranco, S. S. Richmond, M. J. Piovoso, and S. G. BILÉn (2015) "Cognitive Style and Concept Mapping Performance," Journal of Engineering Education, 104(3), pp. 303-325.

[26] Levitt, R. E. (2012) "The Virtual Design Team: Designing Project Organizations as Engineers Design Bridges," Journal of Organization Design, 1(2), p. 14.

[27] Crowder, R. M., M. A. Robinson, H. P. Hughes, and Y. W. Sim (2012) "The development of an agent-based modeling framework for simulating engineering team work," IEEE Transactions on Systems, Man, and Cybernetics Part A:Systems and Humans, 42(6), pp. $1425-1439$.

[28] Bergner, Y., J. J. Andrews, M. Zhu, and J. E. Gonzales (2016) "Agent-Based Modeling of Collaborative Problem Solving," ETS Research Report Series, 2(December).

[29] Perisic, M., T. Martinec, M. Storga, T. Kanduc, M. M. Perišić, T. Martinec, M. ŠTorga, and T. Kanduč (2016) "Agent-Based Simulation Framework To Support Management of Teams Performing Development Activities," Design2016, pp. 1925-1936.

[30] Singh, V., A. Dong, and J. S. Gero (2013) "Social learning in design teams: The importance of direct and indirect communications," Artificial Intelligence for Engineering Design, Analysis and Manufacturing: AIEDAM, 27(2), pp. 167-182. 
[31] FAN, X. and J. YEN (2004) "Modeling and simulating human teamwork behaviors using intelligent agents," Physics of Life Reviews, 1(3), pp. 173-201.

[32] Hulse, D., B. Gigous, K. Tumer, C. Hoyle, and I. Y. Tumer (2017) "Towards a Distributed Multiagent Learning-Based Design Optimization Method," in Volume 2A: 43rd Design Automation Conference, p. V02AT03A008.

[33] Mсcomb, C., J. Cagan, and K. Kotovsky (2015) "Lifting the Veil: Drawing insights about design teams from a cognitively-inspired computational model," Design Studies, 40, pp. 119-142.

[34] Tsvetovat, M. and K. M. Carley (2004) "Modeling Complex Socio-technical Systems using Multi-Agent Simulation Methods," KI, pp. 23-28.

[35] KurtzberG, T. R. (2005) "Feeling creative, being creative: An empirical study of diversity and creativity in teams," Creativity Research Journal.

[36] Hammerschmidt, P. K. (1996), "The Kirton Adaption Innovation Inventory and Group Problem Solving Success Rates," .

[37] Garcia, R. (2005) "Uses of agent-based modeling in innovation/new product development research," Journal of Product Innovation Management, 22(5), pp. 380-398.

[38] Bonabeau, E. (2002) "Agent-based modeling: methods and techniques for simulating human systems." Proceedings of the National Academy of Sciences, 99(suppl. 3), pp. 7280$7287,1709.03423$.

[39] Jennings, N. R. (2000) "On agent-based software engineering," Artificial Intelligence, 117(2), pp. 277-296.

[40] Patrashkova, R. R. and S. A. Mcсомв (2004) "Exploring why more communication is not better: insights from a computational model of cross-functional teams," J. Eng. Technol. Manage, 21, pp. 83-114.

[41] Bernstein, E., J. Shore, and D. Lazer (2018) "How intermittent breaks in interaction improve collective intelligence," Proceedings of the National Academy of Sciences, 115(35), p. 201802407, 1705.07452.

[42] McСomb, C., J. Cagan, and K. Kotovsky (2017) "Optimizing Design Teams Based on Problem Properties: Computational Team Simulations and an Applied Empirical Test," Journal of Mechanical Design, 139(4), p. 041101.

[43] McComb, C. and T. Maier (2018) "Designing Improved Teams for Crowdsourced Competitions," in Volume 7: 30th International Conference on Design Theory and Methodology, ASME, p. V007T06A025.

[44] Hulse, D., K. Tumer, C. Hoyle, and I. Tumer (2018) "Modeling multidisciplinary design with multiagent learning," Artificial Intelligence for Engineering Design, Analysis and Manufacturing: AIEDAM.

[45] Martínez-Miranda, J. and J. Pavón (2012) "Modeling the influence of trust on work team performance," Simulation, 88(4), pp. 408-436.

[46] Zhang, X., L. Luo, Y. Yang, Y. Li, C. M. Schlick, and M. Grandt (2009) "A simulation approach for evaluation and improvement of organisational planning in collaborative product development projects," International Journal of Production Research, 47(13), pp. $3471-3501$. 
[47] Brown, S. L. and K. M. Eisenhardt (1995) PRODUCT DEVELOPMENT: PAST RESEARCH, PRESENT FINDINGS, AND FUTURE DIRECTIONS, Tech. Rep. 2.

[48] Kunz, J. C., T. R. Christiansen, G. P. Cohen, Y. Jin, and R. E. Levitt (1998) A computational simulation model of project organizations that is usable and predictive for routine, project-oriented design tasks, Tech. Rep. 11.

[49] Fernandes, J. V., E. Henriques, A. Silva, and C. Pimentel (2017) "Modelling the dynamics of complex early design processes: an agent-based approach," Design Science, 3, p. e19.

[50] Martinez-Miranda, J., A. Aldea, R. Bañares-Alcantara, and M. Alvarado (2006) TEAKS: Simulation of Human Performance at Work to Support Team Configuration, Tech. rep.

[51] Dehkordi, F. M., A. Thompson, and T. Larsson (2012) "Impacts of Project-Overload on Innovation inside Organizations: Agent-Based Modeling," International Journal of Social, Behavioral, Educational, Economic, Business and Industrial Engineering, 6(11), pp. 2808-2813.

[52] Urban, C. and B. Schmidt (2001) PECS-Agent-Based Modelling of Human Behaviour, Tech. rep.

[53] МсCомв, C., J. Cagan, and K. Kotovsky (2017) "Capturing Human Sequence-Learning Abilities in Configuration Design Tasks Through Markov Chains," Journal of Mechanical Design, 139(9), p. 091101.

[54] Kirkpatrick, S., C. D. Gelatt, and M. P. Vecchi (1983) "Optimization by simulated annealing." Science (New York, N.Y.), 220(4598), pp. 671-80.

[55] Cagan, J. and K. Kotovsky (1997) "Simulated Annealing and the Generation of the Objective Function: A Model of Learning During Problem Solving," Computational Intelligence, 13(4), pp. 534-581.

[56] Yu, B. Y., T. Honda, M. Sharqawy, and M. Yang (2016) "Human behavior and domain knowledge in parameter design of complex systems," Design Studies, 45, pp. 242-267.

[57] Colman, A. M. (2015) A dictionary of psychology, Oxford University Press, USA.

[58] Gere, J. M. and S. P. Timoshenko (2013) Mechanics of Materials.

[59] Vercellone-Smith, P., K. Jablokow, and C. Friedel (2012) "Characterizing communication networks in a web-based classroom: Cognitive styles and linguistic behavior of self-organizing groups in online discussions," Computers and Education, 59(2), pp. 222-235.

[60] He, J., B. S. Butler, and W. R. King (2007) "Team Cognition: Development and Evolution in Software Project Teams," Journal of Management Information Systems, 24(2), pp. 261-292.

[61] Austin-Breneman, J., B. Y. Yu, and M. C. Yang (2015) "Biased Information Passing Between Subsystems Over Time in Complex System Design," Journal of Mechanical Design, 138(1), p. 011101.

[62] Blackburn, J., M. A. Lapre, and L. N. Van Wassenhove (2006) "Brooks' Law Revisited: Improving Software Productivity by Managing Complexity," . 
[63] Applegate, D. L., R. E. Bixby, and V. Chvátal (2007) The Traveling Salesman Problem, Princeton University Press.

[64] Chrysikou, E. G. and R. W. Weisberg (2005) "Following the Wrong Footsteps: Fixation Effects of Pictorial Examples in a Design Problem-Solving Task." Journal of Experimental Psychology: Learning, Memory, and Cognition, 31(5), pp. 1134-1148.

[65] Page, S. E. (2008) The Difference: How the Power of Diversity Creates Better Groups, Firms, Schools and Societies, vol. 88, Princeton University Press.

[66] Collins, B. E. and H. Guetzkow (1964) "A Social Psychology of Group Processes for Decision-Making," Industrial Management Review.

[67] Linsey, J. S., E. F. Clauss, T. Kurtoglu, J. T. Murphy, K. L. Wood, and A. B. MARKMAN (2011) "An Experimental Study of Group Idea Generation Techniques: Understanding the Roles of Idea Representation and Viewing Methods," Journal of Mechanical Design.

[68] Hunt, V., D. Layton, and S. Prince (2015) Diversity matters, Tech. rep., McKinsey \& Company.

[69] Levine, S. S., E. P. Apfelbaum, M. Bernard, V. L. Bartelt, E. J. Zajac, and D. Stark (2014) "Ethnic diversity deflates price bubbles," Proceedings of the National Academy of Sciences, 111(52), pp. 18524-18529.

[70] Nathan, M. and N. Lee (2013) "Cultural diversity, innovation, and entrepreneurship: Firm-level evidence from London," Economic Geography, 89(4), pp. 367-394.

[71] Díaz-García, C., A. González-Moreno, and F. J. Sáez-Martínez (2013) "Gender diversity within $\mathrm{R} \& \mathrm{D}$ teams: Its impact on radicalness of innovation," Innovation: Management, Policy and Practice, 15(2), pp. 149-160.

[72] Phillips, K. W., K. A. Liljenquist, and M. A. Neale (2009) "Is the pain worth the gain? the advantages and liabilities of agreeing with socially distinct newcomers," Personality and Social Psychology Bulletin, 35(3), pp. 336-350.

[73] Pauhus, P. B., M. T. Dzindolet, G. Poletes, and L. M. Camacho (1993) "Perception of Performance in Group Brainstorming: The Illusion of Group Productivity," Personality and Social Psychology Bulletin, 0803973233.

[74] Diehl, M. and W. Stroebe (1987) "Productivity Loss In Brainstorming Groups: Toward the Solution of a Riddle," Journal of Personality and Social Psychology.

[75] Mullen, B., C. Johnson, and E. Salas (1991) "Productivity Loss in Brainstorming Groups: A Meta-Analytic Integration," Basic and Applied Social Psychology, 0803973233.

[76] Steiner, I. D. (1972) Group Processes and Productivity, Academic Press.

[77] MCCARThy, R. (1993) The relationship of individual characteristics of women managers to the pressures experienced at work and choice of coping strategy, Phd thesis, University of Hertfordshire.

[78] Smith, K. G., K. A. Smith, J. D. Olian, H. P. Sims, D. P. O’Bannon, and J. A. Scully (1994) "Top Management Team Demography and Process: The Role of Social Integration and Communication," Administrative Science Quarterly, 39(3), p. 412, arXiv: $1011.1669 v 3$. 
[79] Di Penta, M., M. Harman, G. Antoniol, and F. Qureshi (2007) "The effect of communication overhead on software maintenance project staffing: A search-based approach," in IEEE International Conference on Software Maintenance, ICSM, pp. 315-324. 


\section{Sam Lapp \\ sam.m.lapp@gmail.com \\ samlapp.com}

Education M.S. Engineering Design

May 2019

The Pennsylvania State University

GPA: 4.00

B.S. in Engineering Science

May 2017

The Pennsylvania State University, Schreyer Honors College

GPA: 3.97

Projects Reducing Post Harvest Loss of Produce in Kenya

Developing venture to reduce loss of fresh produce in supply chain

Spring 2018-Present

Prototyping apps to connect farmers and market sellers

Kisumu, Kenya

Travelled to Kisumu, Kenya to meet and learn from customers

Dynamic Alignment Tool for Lower-Limb Prosthetics

Designed tool for quantifying process of aligning a prosthesis

Oct 2017- March 2018

Built prototypes to measure foot-pressure distribution and leg motion

Collaborated with team in South Africa and Germany

Undergraduate Thesis in Engineering Science and Music

2016-2017

Examined tuning systems of non-Western culture

State College, PA

Evaluated computational models for predicting perception of dissonance

Developed tool for analyzing cross-cultural patterns in musical phrases

Community Grooves to Go: mobile music studio

Building a mobile music venue and recording studio

2017-Present

Facilitating co-creation of music through writing and recording workshops

Participating in Emerging City Champions Fellowship

House Manager at The Co.Space (co-living house)

2018-2019

Facilitating community experiences for residents (e.g. house retreat)

State College, PA

Organizing and running events and special programming (e.g. house concerts)

Fostering community partnerships (e.g. with local innovation accelerators)

\section{Work}

Agent Based Modeling of Engineering Teams (Thesis Research)

Research Assistant in THRED Lab with Dr. Chris McComb

Summer 2018-present

Developing agent-based models to simulate team problem solving

Validating models with empirical experiments

Machine Learning for Population Health Data (Research)

Center for Health Organization Transformation

Fall 2017 - Spring 2018

PSU CHOT Lab

Developed machine learning models for large, sparse data sets

Clustered patients based on data from Electronic Medical Records

Pipelines for Cross-Domain Knowledge Transfer (REU Internship)

Human Computer Interaction Department

Designed crowd-sourced platform for knowledge transfer

Explored computational methods for finding analogies in literature

Carnegie Mellon University

Parser for Internet of Things Data (Internship)

Summer 2015

Interned at Wipro Technologies in India

Bangalore, India

Developed a flexible, generic parser for processing data

Accommodated data from current and future devices and formats 